Portland State University

PDXScholar

9-14-1993

\title{
A Study of Spiking and Relaxation Oscillations in Nd:YAG Laser Using Measured Laser Parameters
}

\author{
Ramesh K. Shori \\ Portland State University
}

Follow this and additional works at: https://pdxscholar.library.pdx.edu/open_access_etds

Part of the Electrical and Computer Engineering Commons Let us know how access to this document benefits you.

\section{Recommended Citation}

Shori, Ramesh K., "A Study of Spiking and Relaxation Oscillations in Nd:YAG Laser Using Measured Laser Parameters" (1993). Dissertations and Theses. Paper 4641.

https://doi.org/10.15760/etd.6525

This Thesis is brought to you for free and open access. It has been accepted for inclusion in Dissertations and Theses by an authorized administrator of PDXScholar. Please contact us if we can make this document more accessible: pdxscholar@pdx.edu. 
AN ABSTRACT OF THE THESIS OF Ramesh K. Shori for the Master of Science in Electrical and Computer Engineering presented September 14, 1993.

Title: A Study of Spiking and Relaxation Oscillations in Nd:YAG Laser Using Measured Laser Parameters.

APPROVED BY THE MEMBERS OF THE THESIS COMMITTEE:
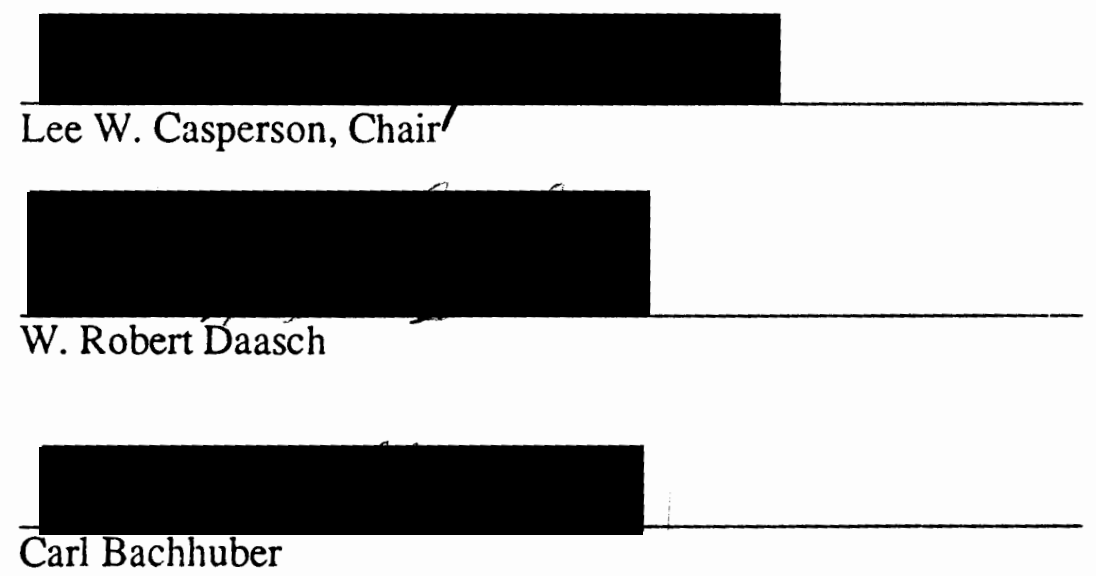

It was shown analytically and experimentally that when the cavity losses are perturbed, the output intensity experiences an amplitude modulation or becomes a regular train of spikes, with the frequency depending on both the frequency of perturbation as well as pump power. Coupled nonlinear rate equations including the cavity perturbation term, are solved numerically by a Runga-Kutta method using experimentally-measured parameter values for Nd:YAG laser. A continuously pumped Nd:YAG laser was used to verify this theory. 
A STUDY OF SPIKING AND RELAXATION OSCILLATIONS IN Nd:YAG

LASER USING MEASURED LASER PARAMETERS

by

RAMESH K. SHORI

A thesis submitted in partial fulfillment of the requirements for the degree of

MASTER OF SCIENCE

in

ELECTRICAL AND COMPUTER ENGINEERING

Portland State University

1993 
TO THE OFFICE OF GRADUATE STUDIES:

The members of the committee approve the thesis of Ramesh K. Shori presented September 14, 1993.

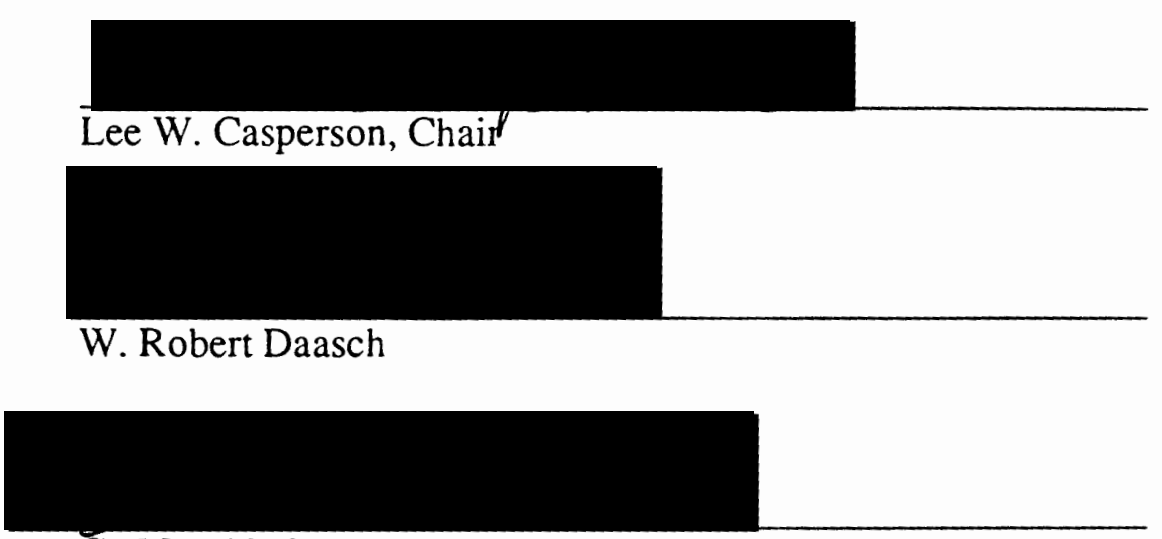

Carl Bachhuber

APPROVED:
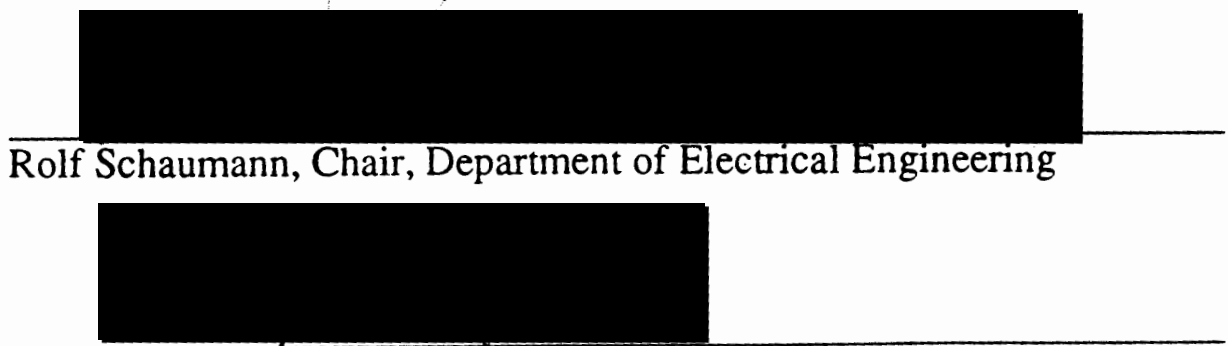

Roy W. Koch, fice Provost for Graduate Studies and Research 
DEDICATED TO THE MEMORIES OF PURAN CHAND SHORI 


\section{ACKNOWLEDGMENTS}

For any major undertaking to be successful requires the assistance from others. And the completion of this thesis is no exception.

I would like to thank Dr. Oscar Stafsudd at the Electrical Engineering Department at UCLA for providing the laboratory space and the equipment which enabled me to complete this project. I am grateful to Dr. Stafsudd for providing the day to day guidance throughout my stay in his lab and for making the frequent Saturday morning visits to lab in the beginning stages of this work. I would also like to thank James Ho, Andy Walston, Dorothy Lai, Debbie Vickers, Meng Chiao, Phyllis Nelson for their technical assistance and support, and more importantly, their patience putting up with the inconveniences when I was running the $28 \mathrm{hr}$ simulations (on ALL the computers!), when the lab had to be cooled to $50 \mathrm{~F}$, and many other idiosyncrasies. I am especially grateful to James Ho for his assistance with writing the Basic program and in taking measurements during the all nighters; to Andy Walston for the many meaningful and often frustrating discussions in analyzing the data and editing this thesis; to Dorothy Lai for her time and effort in typing the thesis especially with all the equations.

I would like to thank Dr. Casperson at the Electrical Engineering Dept. at Portland State University for being my advisor and allowing me to have this rather unusual arrangement of being a student in his lab and yet doing all the work at UCLA some thousand miles away. I would also like to thank Drs. Daasch and Bachhuber for taking the time to serve on my committee. 
Last, but not least, I would like to thank my family, especially Raj, for their support and encouragement during the difficult time when going back and forth between Portland and Los Angeles on the weekly basis. Without their help this work would not have been possible. 
TABLE OF CONTENTS

PAGE

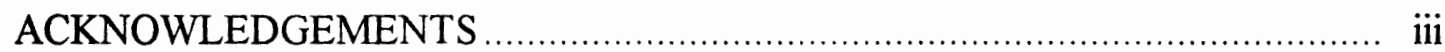

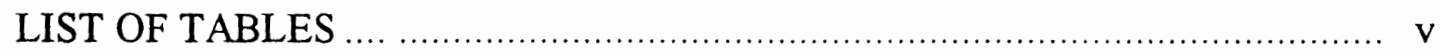

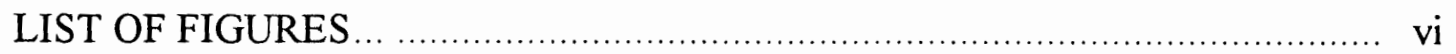

\section{CHAPTER}

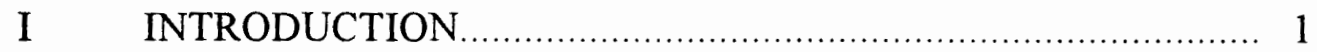

II THEORETICAL ANALYSIS ............................................... 3

Transient Analysis ....................................................... 7

Spiking …..................................................... 7

Relaxation Oscillations ....................................... 9

Numerical Analysis................................................. 11

III EXPERIMENTAL RESULTS …..................................... 42

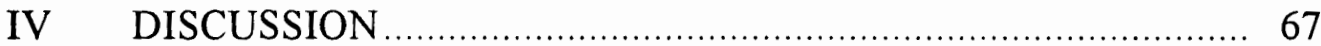

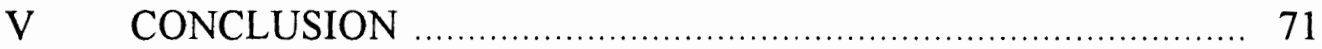

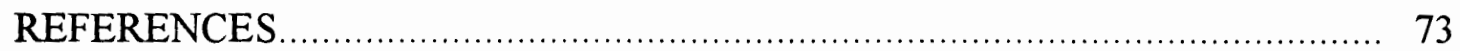

\section{APPENDICES}

A CALCULATION OF BEAM DIAMETER ................................ 74

B THE DETERMINATION OF THE DISTRIBUTED CAVITY

LOSS $\alpha_{\mathrm{T}}$ AND THE GAIN $\gamma_{0}$ PER ROUND TRIP …................. 79

C BOLTZMANN DISTRIBUTION OF ATOMS IN Nd:YAG ......... 93 
D LINEARIZED RATE EQUATIONS .................................. 96

E NUMERICAL SOLUTION TO THE RATE EQUATION ........... 104 


\section{LIST OF TABLES}

TABLE PAGE

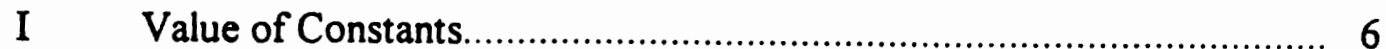




\section{LIST OF FIGURES}

FIGURE

PAGE

1. Simplified energy level diagram of a four level laser [5] ......................... 13

2. Absorption spectrum of Nd:YAG laser, $P_{\text {in }}=225 \mathrm{~mW} \ldots \ldots \ldots \ldots \ldots \ldots \ldots \ldots \ldots \ldots \ldots \ldots \ldots \ldots . . .13$

3. Computer simulation of the spiking behavior in Nd:YAG laser ............... 14

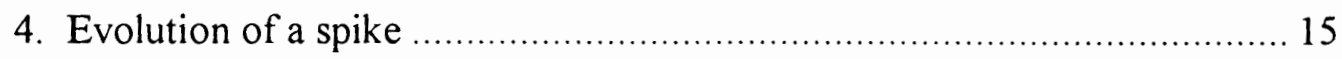

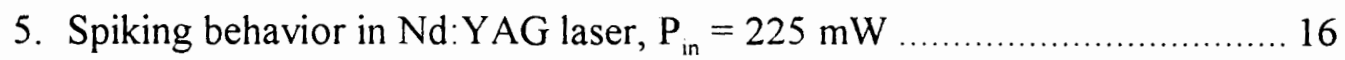

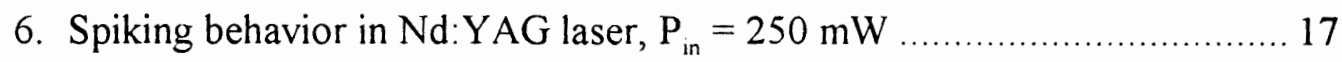

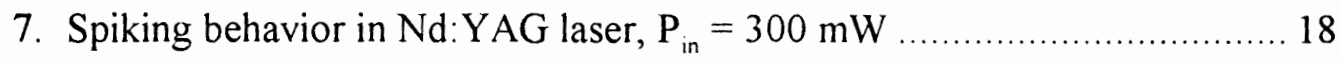

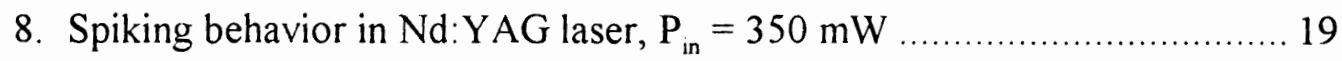

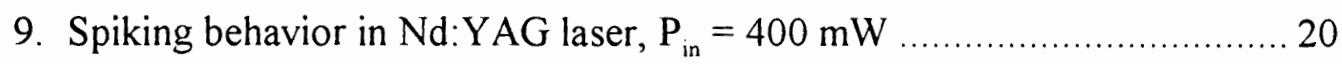

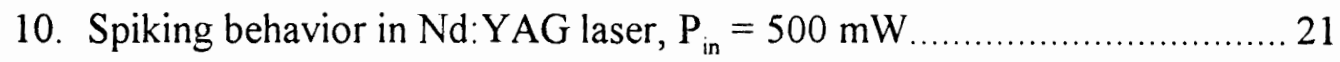

11. Change in modulation depth and the resonant frequency as funtion of $P_{\text {in }} 22$

12A. Spiking and relaxation oscillations behavior in Nd:YAG,

$$
P_{\text {in }}=225 \mathrm{~mW} \text {. }
$$

12B. Details of the relaxation oscillation at $f_{R-O}=16.485 \mathrm{kHz}$.

13A. Spiking and relaxation oscillations behavior in Nd:YAG,

$$
P_{\text {in }}=250 \mathrm{~mW} \text {. }
$$

13B. Details of the relaxation oscillation at $\mathrm{f}_{\mathrm{R}-\mathrm{O}}=25.187 \mathrm{kHz}$.

14A. Spiking and relaxation oscillations behavior in Nd:YAG,

$$
P_{\text {in }}=300 \mathrm{~mW} \text {. }
$$

14B. Details of the relaxation oscillation at $\mathrm{f}_{\mathrm{R} \cdot \mathrm{C}}=36.874 \mathrm{kHz}$. 28 
15A. Spiking and relaxation oscillations behavior in Nd:YAG,

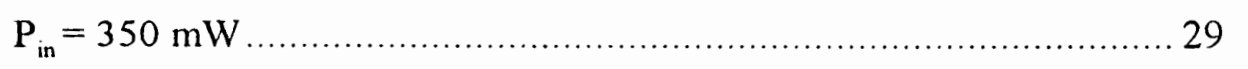

15B. Details of the relaxation oscillation at $\mathrm{f}_{\mathrm{R}-\mathrm{O}}=45.618 \mathrm{kHz} \ldots \ldots \ldots \ldots \ldots \ldots . \ldots . \ldots . \ldots$

16A. Spiking and relaxation oscillations behavior in Nd:YAG,

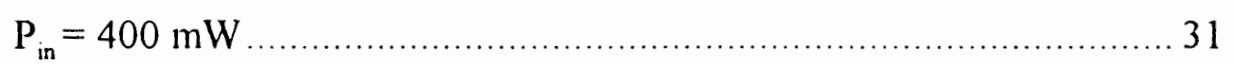

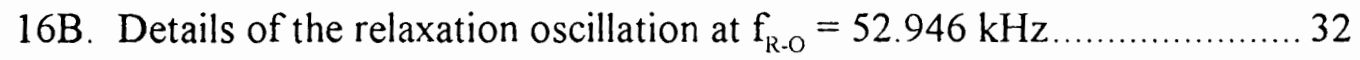

17A. Spiking and relaxation oscillations behavior in Nd:YAG,

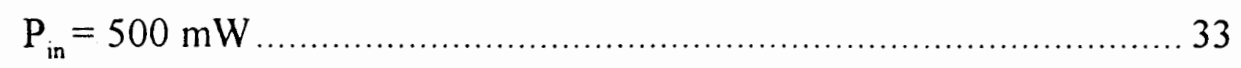

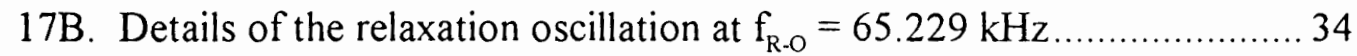

18. Spiking and giant pulsing in Nd:YAG laser .................................... 35

19. Initiation of giant pulses from steady state output .............................. 36

20. Details of the gint pulses and the cavity loss ..................................... 37

21A. Laser response to cavity loss modulated at the first subharmonic of the

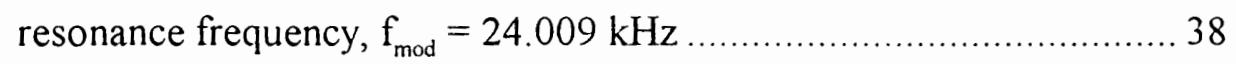

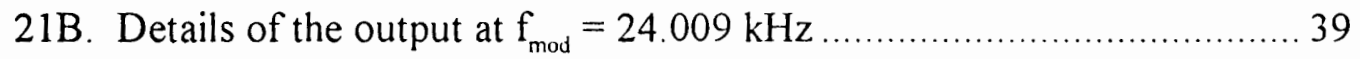

22A. Laser response to cavity loss modulated at the second subharmonic of

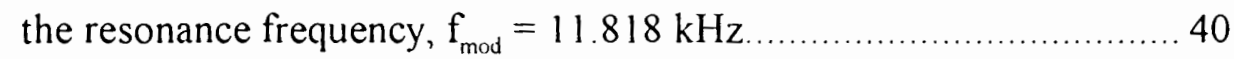

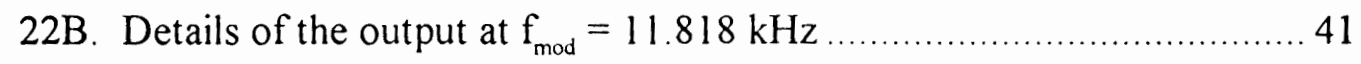

23A. Schematic diagram of the experimental setup used to study the relaxation

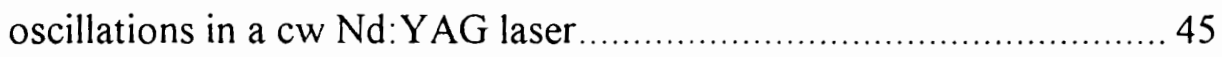

23B. The CVI laser used for all data acquisition ...................................... 45

24. Relaxation oscillation response of $\mathrm{Nd}$ : $Y A G$ laser pumped at $87.477 \mathrm{~V}_{\mathrm{DC}}$ and at $11.26 \mathrm{~A}$ (flashlamp voltage adn current), $\mathrm{P}_{\text {in }}=\mathrm{IV}=985 \mathrm{~W} \ldots 46$

25. Laser output when pumped at $V_{\text {in }}=88.012 \mathrm{~V}, I_{\text {in }}=11.32 \mathrm{~A}$ which corresponds to $P_{\text {in }}=996.3 \mathrm{~W}$. 
26. Laser output when pumped at $\mathrm{V}_{\text {in }}=89.007 \mathrm{~V}, \mathrm{I}_{\text {in }}=11.38 \mathrm{~A}$ which corresponds to $P_{\text {in }}=1012.9 \mathrm{~W}$ 48

27. Laser output when pumped at $\mathrm{V}_{\text {in }}=89.996 \mathrm{~V}, \mathrm{I}_{\mathrm{in}}=11.44 \mathrm{~A}$ which corresponds to $P_{\text {in }}=1029.55 \mathrm{~W}$ 49

28. Laser output when pumped at $\mathrm{V}_{\text {in }}=91.006 \mathrm{~V}, \mathrm{I}_{\text {in }}=11.52 \mathrm{~A}$ which corresponds to $\mathrm{P}_{\text {in }}=1048.39 \mathrm{~W}$ 50

29. Laser output when pumped at $\mathrm{V}_{\text {in }}=92.003 \mathrm{~V}, \mathrm{I}_{\text {in }}=11.57 \mathrm{~A}$ which corresponds to $\mathrm{P}_{\text {in }}=1058.22 \mathrm{~W}$ 51

30. Laser output when pumped at $\mathrm{V}_{\text {in }}=93.008 \mathrm{~V}, \mathrm{I}_{\mathrm{in}}=11.65 \mathrm{~A}$ which corresponds to $\mathrm{P}_{\text {in }}=1083.54 \mathrm{~W}$ 52

31. Laser output when pumped at $\mathrm{V}_{\mathrm{in}}=94.013 \mathrm{~V}, \mathrm{I}_{\mathrm{in}}=11.72 \mathrm{~A}$ which corresponds to $P_{\text {in }}=1101.83 \mathrm{~W}$

32. Laser output when pumped at $\mathrm{V}_{\text {in }}=95.008 \mathrm{~V}, \mathrm{I}_{\text {in }}=11.79 \mathrm{~A}$ which corresponds to $\mathrm{P}_{\text {in }}=1120.14 \mathrm{~W}$

33. Laser output when pumped at $\mathrm{V}_{\text {in }}=96.002 \mathrm{~V}, \mathrm{I}_{\text {in }}=11.86 \mathrm{~A}$ which corresponds to $\mathrm{P}_{\mathrm{in}}=1138.58 \mathrm{~W}$

34. Laser output when pumped at $\mathrm{V}_{\text {in }}=97.006 \mathrm{~V}, \mathrm{I}_{\text {in }}=11.93 \mathrm{~A}$ which corresponds to $P_{\text {in }}=1157.28 \mathrm{~W}$ 56

35. Laser output when pumped at $\mathrm{V}_{\text {in }}=98.025 \mathrm{~V}, \mathrm{I}_{\text {in }}=11.99 \mathrm{~A}$ which corresponds to $P_{\text {in }}=1175.32 \mathrm{~W}$

36. Laser output when pumped at $\mathrm{V}_{\text {in }}=99.018 \mathrm{~V}, \mathrm{I}_{\mathrm{in}}=12.05 \mathrm{~A}$ which corresponds to $\mathrm{P}_{\text {in }}=1193.17 \mathrm{~W}$ 58

37. Laser output when pumped at $\mathrm{V}_{\text {in }}=100.008 \mathrm{~V}, \mathrm{I}_{\text {in }}=12.12 \mathrm{~A}$ which corresponds to $P_{\text {in }}=1212.10 \mathrm{~W}$ 59

38. The change in resonance frequency of the relaxation osccillation as a function of pump power. 60 
39. Relaxation oscillation response of Nd:YAG laser pumped at $85.521 \mathrm{~V}$,

$$
I_{\text {in }}=11.23 \mathrm{~A}, P_{\text {in }}=960.38 \mathrm{~W} ; P_{\text {out }}=7 \mathrm{~mW}, f_{\mathrm{R}-\mathrm{O}}=22.07 \mathrm{kHz} \text {.. }
$$

40. The laser response with $V_{\text {in }}=185.52 \mathrm{~V}, I_{\text {in }}=11.23 \mathrm{~A}, P_{\text {in }}=960.38 \mathrm{~W} \ldots 62$

41. The laser response with $V_{\text {in }}=185.52 \mathrm{~V}, \mathrm{I}_{\text {in }}=11.23 \mathrm{~A}, \mathrm{P}_{\text {in }}=960.38 \mathrm{~W} \ldots .63$

42. The laser response with $\mathrm{V}_{\text {in }}=185.52 \mathrm{~V}, \mathrm{I}_{\text {in }}=11.23 \mathrm{~A}, \mathrm{P}_{\text {in }}=960.38 \mathrm{~W} \ldots 64$

43. The laser response with $V_{\text {in }}=185.52 \mathrm{~V}, I_{\text {in }}=11.23 \mathrm{~A}, P_{\text {in }}=960.38 \mathrm{~W} \ldots .67$

44. The laser response with $\mathrm{V}_{\text {in }}=185.52 \mathrm{~V}, \mathrm{I}_{\text {in }}=11.23 \mathrm{~A}, \mathrm{P}_{\mathrm{in}}=960.38 \mathrm{~W} \ldots .68$

45. Comparison of the experimental and the theoretical relaxation oscillation values as a funtion of pump power 


\section{CHAPTER I}

\section{INTRODUCTION}

Using linearized rate equations, McCumber in 1966 predicted that a perturbation in the intracavity intensity of a four-level laser would results in an output power spectrum that contains a resonant spike whose frequency is dependent on the pump power [1]. For almost a decade after the theory was introduced, literature was still being published testing the validity of McCumber's theory. Geusic et.al. presented results using a continuous wave (cw) Nd:YAG laser which qualitatively showed that the output contained a resonant peak [2]. Similar behavior was also reported by Ikegami and Suematsue using an injection laser, where they showed that when the injection current is modulated at microwave frequencies, the output modulation depth exhibits a resonant peak. Kimura and Otsuka, also using a cw pumped Nd:YAG laser, reported that when the cavity is perturbed sinusiodally, the output intensity experiences a sinusoidal amplitude modulation or becomes a regular train of spikes, depending on the frequency of the perturbation. They term the former output response as a resonant AM mode and the latter output response as a spiking mode. They claim that while the frequency of the resonant AM mode is pump power and cavityparameter dependent and coincides with McCumber's theory, the frequency of the spiking mode is independent of pump power and cavity losses and depends only on the fluorescent lifetime of the laser medium.

In this thesis, it is shown analytically and experimentally for the case of a cw pumped Nd:YAG laser that, when the cavity losses are perturbed, the output intensity ex- 
periences an amplitude modulation or becomes a regular train of spikes, with the frequency depending on the pump power. Nonlinear rate equations including the cavity perturbation term, are solved numerically by a Runga-Kutta method using experimentally measured parameter values for a Nd:YAG laser.

A flash lamp-pumped Nd:YAG laser was used to verify this theory. The intracavity loss modulation was accomplished by placing an acousto-optic modulator within the laser cavity. For a proper choice of loss modulation frequency and pump power, the laser output shows the periodic spiking behavior predicted by numerical analysis of the nonlinear rate equations. 


\section{CHAPTER II}

\section{THEORETICAL ANALYSIS}

The dynamic as well as the steady state behavior of a laser can be described with reasonable precision by a set of coupled nonlinear rate equations. The Nd:YAG laser used in the present work will be described in terms of the energy-level diagrams shown in Figure 1. In any laser, two energy levels are of prime importance in laser action: the excited upper laser level $E_{2}$, and the lower laser level $E_{1}$. While many analysis of laser action in three- and four-level systems can be carried out to a good approximation using a simplified two-level representation, the present work will solve the rate equations for a general four-level case while making as few assumptions as possible. The rate equations for a four-level laser are:

$$
\begin{aligned}
& \frac{d N_{0}}{d t}=\frac{N_{1}}{\tau_{10}}+\frac{N_{2}}{\tau_{20}}+\frac{N_{3}}{\tau_{30}}-P \\
& \frac{d N_{1}}{d t}=I_{21} \sigma_{21}\left(N_{2}-N_{1}\right)+\frac{N_{2}}{\tau_{21}}+\frac{N_{3}}{\tau_{31}}-\frac{\left(N_{1}-N_{1 B}\right)}{\tau_{10}} \\
& \frac{d N_{2}}{d t}=-I_{21} \sigma_{21}\left(N_{2}-N_{1}\right)-\left(N_{2}-N_{28}\right)\left(\frac{1}{\tau_{20}}+\frac{1}{\tau_{21}}\right)+\frac{N_{3}}{\tau_{32}} \\
& \frac{d N_{3}}{d t}=P-\left(N_{3}-N_{3 B}\right)\left(\frac{1}{\tau_{30}}+\frac{1}{\tau_{31}}+\frac{1}{\tau_{32}}\right) \\
& \frac{d I_{21}}{d t}=I_{21} \sigma_{21}\left(N_{2}-N_{1}\right) \frac{c}{n V_{m}}-\frac{I_{21}}{\tau_{\text {cav }}}+S .
\end{aligned}
$$


The "N's" represent the population of each level in absolute numbers, rather than in terms of population density (as commonly seen in the literature). Since the ground state population $\mathrm{N}_{0}$ is orders of magnitude larger than $N_{1}, N_{2}$, or $N_{3}$, (1) can be set equal to zero because $\mathrm{N}_{0}$ does not change appreciably with time under normal operation of the laser. $N_{1 B}, N_{2 B}$, and $N_{3 B}$ are the steady-state (un-pumped) equilibrium populations for the respective levels due to the Boltzmann distribution. The value of these populations are calculated in Appendix C. $\mathrm{N}_{2 \mathrm{~B}}$ and $\mathrm{N}_{3 \mathrm{~B}}$ are for all intents purposes zero, and only $\mathrm{N}_{\mathrm{LB}}$ need be retained for an accurate analysis.

The " $\tau$ 's" are the relaxation or decay time constants for atoms as they undergo transition from one level to another. The value for $\tau_{31}$ is not available in the literature to date. But it is generally accepted in the laser community that $\tau_{31}$ is much longer than any other time constant and, therefore, that it is safe to eliminate any terms involving $\tau_{31}[5]$. $\tau_{c w}$ is the average lifetime of a photon in the resonator before being scattered, emitted, or lost in other ways to the optical system. $\tau_{\infty}$ is defined as

$$
\tau_{\text {aw }}=\frac{\tau_{\text {RT }}}{\text { RTloss }}=\frac{2 \mathrm{~L} / \mathrm{c}}{\text { RTloss }}
$$

where $\tau_{\mathrm{RT}}$ is the round-trip time of a photon in a resonator of optical path length $L$, and $\mathrm{RT}$ loss is the total round trip loss in the resonator, including the mirror losses (see Appendix B for a detailed calculation of $\alpha_{\mathrm{T}}$, the total losses prorated over the length of the gain medium, 1). It should be pointed out that the measurements of the time constants is no trivial matter, and in some cases, like that of $\tau_{31}$, the value is not even available. Consequently, instead of having one numerical value for any given time constant, the literature contains a range of values. Similar argument is also true for values of the stimulated emission cross-section area, $\sigma_{21} . I_{21}$ is the intracavity photon flux (units: \# photons/sec$\left.\mathrm{cm}^{2}\right)$. P represents the pump rate at which atoms are transferred from the ground state to the pump band (units: \# photons/sec). While the experiment was performed using a laser 
pumped by tungsten lamps with a broad emission spectrum, the theoretical analysis will be carried out for a laser pumped by a laser diode that emits photons in a very narrow spectral range, near $808 \mathrm{~nm}$, which overlaps with the absorption spectrum of Nd:YAG (Figure 2). For the purpose of comparing experimental and theoretical results, Appendix B also includes a calculation that attempts to estimate how much of the energy from the tungsten lamps is contained in the $808 \mathrm{~nm}$ band.

In the current analysis, it is assumed that all the pump photons are absorbed by the YAG rod and therefore directly contribute towards increasing the population of level $3, \mathrm{~N}_{3} . \mathrm{V}_{\mathrm{m}}$ is the mode volume (see Appendix A). The term $S$ in (5) accounts for the increase in the intracavity intensity due to small amount of spontaneous emission. Although $S$ is very small (almost zero), this term is included because it provides the source of radiation which initiates laser emission. For practical analysis which follows $S$ is set equal to zero. The numerical values of all constants used and the references from which the values were taken are listed in Table I. Equations 2-5 represents a series of four equations and four unknowns. The steady state solutions to these equations are:

$$
\begin{aligned}
& N_{1}=\left[P \frac{\tau_{3}}{\tau_{32}} \cdot \tau_{20}+N_{1 P} \frac{\tau_{20}}{\tau_{10}}-\Delta N_{c}\right] \cdot \frac{\tau_{10}}{\tau_{10}+\tau_{20}} \\
& N_{2}=P \frac{\tau_{3}}{\tau_{32}} \cdot \tau_{20}-\left[P \frac{\tau_{3}}{\tau_{32}} \cdot \tau_{20}+N_{13} \frac{\tau_{20}}{\tau_{10}}-\Delta N_{c}\right] \cdot \frac{\tau_{20}}{\tau_{10}+\tau_{20}} \\
& N_{3}=P \tau_{3} \\
& I_{21}=\left(\frac{N_{3}}{\tau_{32}}-\frac{N_{2}}{\tau_{2}}\right) \cdot \frac{\tau_{00 \mathrm{C}}}{n V_{m}}
\end{aligned}
$$

where $\Delta \mathrm{N}_{\mathrm{c}}$ is the steady state population inversion value. 
TABLE I

VALUE OF CONSTANTS

- Optical Path Length: OPL $=32.77 \mathrm{~cm}$

- Index of YAG: $n=1.82$ [7]

- Mode Area: $A_{m}=4.2429 * 10^{-3} \mathrm{~cm}^{2}$

- Mode Volume: $\mathrm{V}_{\mathrm{m}}=2.5457 * 10^{-2} \mathrm{~cm}^{3}$

- Doping Level of $\mathrm{Nd}^{+3}: 1.38 * 10^{20}$ atoms $/ \mathrm{cm}^{3}$

- Population of Ground State: $\mathrm{N}_{0}=\left[\mathrm{Nd}^{+3}\right] * \mathrm{~V}_{\mathrm{m}}=3.513 * 10^{18}$

- Stimulated Emission Cross Section Area: $\sigma_{21}=6.5 * 10^{-19} \mathrm{~cm}^{2}$ [7]

- Time Constants:

$$
\begin{aligned}
& \tau_{10}=11 \mathrm{~ns} \\
& \tau_{20}=395 \mu \mathrm{s} \\
& \tau_{21}=550 \mu \mathrm{s} \\
& \tau_{30}=50 \mu \mathrm{s} \\
& \tau_{32}=450 \mathrm{ps} \\
& \tau_{2}=\left(\tau_{2^{-1}}+\tau_{21}-1\right)^{-1}=230 \mu \mathrm{s} \\
& \tau_{3}=\left(\tau_{30}+1+\tau_{32}\right)^{-1}=0.449996 \mathrm{~ns} \\
& \tau_{\mathrm{RT}}=(2 * \mathrm{OPL}) / \mathrm{c}=2.18467 \mathrm{~ns}
\end{aligned}
$$

- $h v_{808}=2.46015 * 10^{-19} \mathrm{~J}$

- $h v_{1064}=1.86823 * 10^{-19} \mathrm{~J}$

Almost always, one is interested in knowing the value of the steady state output power of a laser. To calculate the value of the steady state output power $P_{\text {ou }}$ multiply the intracavity intensity in (10) by the transmission of the output coupler, the mode area of the beam $A_{m}$, the energy per photon of the photon emitted at $\lambda=1.064 \mu \mathrm{m}$ and a factor of $1 / 2$. The $I_{21}$ calculated in (10) is the sum of the intensity of the right and left traveling wave inside the cavity. Since we are only interested in the output from one side of the la- 
ser, the value of $I_{21}$ needs to by divided by a factor of 2 . In equation form, $P_{\text {out }}$ can be expressed as

$$
P_{o x}=\frac{1}{2}\left(1-R_{o c}\right) \cdot A_{m} \cdot h V_{1064} \cdot I_{21} .
$$

The rate equations were solved numerically in a Quick Basic program using a Runga-Kutta method and experimentally measured parameters to study the transient or dynamic behavior of laser oscillations. A listing of the basic program is included in Appendix $\mathrm{E}$. The transient behavior is discussed in the next section.

\section{Transient Analysis}

In this section some aspects of the transient or dynamic behavior of a laser oscillator are discussed. While lasers can exhibit several different kinds of characteristic transient or modulation behaviors, the discussion here will be limited to spiking and relaxation oscillation. These two types of behaviors are the most predominant mechanisms that cause fluctuations in the output of many lasers, especially solid state lasers in which the upper-state lifetime is relatively long compared to the laser cavity decay time $\tau_{c a v}$. Following Siegman's notation [11], the term "spiking" will be used to describe the discrete, sharp, large-amplitude pulses that typically occur during the initial turn on phase, or when the laser gain or loss is modulated at some resonant frequency. The term "relaxation oscillation" will then be used to describe small-amplitude, quasi-sinusoidal, exponentially damped oscillations about the steady state amplitude which occur when a cw output laser is suddenly perturbed by any kind of small fluctuations in the cavity gain or loss, or cavity alignment.

Spiking. The phenomenon of the spike formation when a Nd:YAG laser is first turned on can be explained with the aid of results from a computer simulation shown in Figures 3 and 4. Figure 3 shows the changes in the population inversion and the onset of 
the spiking behavior when the laser is first turned on. Figure 4 is a more detail graph of the changes in the population inversion and the first few spikes. While the graph shows the change in output power $\mathrm{P}_{\text {out }}$ as function of time, the following discussion will be in terms of change in circulation intensity $I_{21}$ since $I_{21} \propto P_{\text {our }}$.

When the laser pump source is first turned on, the number of photons per frequency corresponding to $\lambda=1.064 \mu \mathrm{m}$ is essentially zero. The pump radiation causes a linear buildup of the excited atom and the population is inverted. Although under steady state oscillation conditions the population inversion $\left(\mathrm{N}_{2}-\mathrm{N}_{1}\right)$ can never exceed the threshold value of $\left(\mathrm{N}_{2}-\mathrm{N}_{1}\right)_{t \rightarrow}$, under transient conditions the pumping can raise the population inversion above the threshold level because not enough laser oscillation has been built-up and no radiation due to stimulated emission yet exists to pull the population inversion back down.

As soon as the population inversion passes the threshold inversion, the net round trip laser gain exceeds the loss and the circulating photon flux $I_{21}$ in the cavity begins to build up exponentially from noise. The increase in $I_{21}$ continues until the level of $I_{21}$ is substantially higher than the steady state value $I_{21}$ for the particular pump level. But when $I_{21}$ becomes very large, the rate of depletion of the population inversion due to stimulation emission becomes correspondingly large and is greater than the pumping rate P. Consequently, the population inversion passes through a maximum and begins to decrease rapidly, driven downward by the large circulating intensity flux $I_{21}$. The point at which the population inversion just reaches the steady state or threshold value $\left(N_{2}-N_{1}\right)_{t}$ is also the point at which the photon flux $I_{21}$ reaches its peak value. Since, $I_{21}$ is still very large, the population inversion continues to be driven below its threshold value until the net gain of the laser is less than the net loss of the cavity and $I_{23}$ decreases exponentially. When $I_{21}$ reaches the threshold value $I_{21 \text { w }}$, the population inversion reaches a minimum, after which the pump P can again begin to build up the population inversion towards 
threshold. The photon flux $I_{21}$ continuous to decrease to negligible values, however, such that the pumping back up of the population inversion is essentially independent of the photon flux [11].

Because of the rapid rates of rise and fall of the photon flux $\mathrm{I}_{21}$, the laser spikes are steep and narrow. This kind of large-signal spiking behavior exponentially dampens towards steady state or quasi-sinusoidal relaxation-oscillation type of behavior because neither the cavity photon flux $I_{21}$ nor the population inversion decreases completely to zero following a spike. Consequently, each successive spike starts from initial conditions that are closer and closer to the steady state behavior of the laser. Figures 5 through 10, generated using the basic program, illustrate the onset of spiking behavior and the subsequent exponential decay to a steady state value for a range of pump powers.

Once the spiking behavior in a laser has damped down to what are essentially small-amplitude fluctuations about the steady state oscillation conditions in the laser, we are now in a position to carry out a linearized small signal analysis of the laser equations. This topic is taken up in the next section.

Relaxation Oscillations Small signal analysis by definition implies that the value of the perturbations of all variables are small with respect to the steady state values. For the purpose of analyzing relaxation oscillations, the perturbations can be introduced in the form of cavity loss or gain modulation. Since the perturbations are going to be small with respect to the steady state value, the response of the laser to either type of modulation should be the same [1]. In this section, the perturbation will be in the form of gain modulation. For subsequent theoretical and experimental analysis the perturbation will be introduced as loss modulation. Assume that the solutions to the rate equations (2)-(5) are of the form

$$
\begin{aligned}
& N_{1}=\bar{N}_{1}+\operatorname{Re}\left(\Delta N_{1} e^{-j \omega t}\right) \\
& N_{2}=\bar{N}_{2}+\operatorname{Re}\left(\Delta N_{2} e^{-j \omega t}\right)
\end{aligned}
$$




$$
\begin{aligned}
& N_{3}=\bar{N}_{3}+\operatorname{Re}\left(\Delta N_{3} e^{-j \omega t}\right) \\
& I_{21}=I D c+\operatorname{Re}\left(\Delta I_{21} e^{-j \omega t}\right)
\end{aligned}
$$

where the first term in each solution represents the steady state values for that variable, and the second term is a small perturbation around the steady state value. To allow for any phase differences between the perturbation and the signal, the second term in each of the above solutions includes an $\mathrm{e}^{-\mathrm{jox}}$ term. In addition to the above solutions, assume that the pumping also deviates from a steady state value in the same manner, i.e.:

$$
P=\bar{P}+\operatorname{Re}\left(\Delta P^{-j \omega t}\right) \text {. }
$$

Let us introduce the term modulation depth $\xi$ to define the relative output intensity fluctuation as a function of modulation frequency for different pump powers. The mathematics necessary to drive an analytical expressions of the modulation depth as a function of modulation frequency is presented in Appendix D, and only the final result is given here. To evaluate the change in the intensity as a function of the pump fluctuation a Mathcad program (DeltaI5.MCD) was written and is included in Appendix D. The expression for the modulation depth $\xi$ as a function of modulation frequency is

$$
\xi=\frac{|\Delta \mathrm{I}|}{\mathrm{I}}=\frac{\left(\Delta \mathrm{N}_{2}-\Delta \mathrm{N}_{1}\right)}{\tau_{\mathrm{con}} \Delta \mathrm{N}_{\mathrm{c}} \omega}
$$

In (17), note that the oscillation frequency $\omega$ is strongly dependent on the photon flux IDC and therefore, on the pump power density. This dependence is confirmed in the results from the Mathcad program (fig. 11) which show that the modulation depth is peaked sharply about a characteristic modulation frequency whose value depends strongly on the pump power and the time constants. Note that when the modulation frequency approaches some resonance or characteristic frequency, the modulation depth is greater than unity. Under these conditions, the small signal analysis no longer applies, and one must resort to iterative numerical analysis of the original coupled nonlinear rate equations. This topic is taken up in the next section. 


\section{Numerical Analysis of the Rate Equations}

In this section the nonlinear rate equations are again solved numerically using the same basic program used in the section on Spiking. But, this time the equations include a cavity perturbation term. Once the output of the laser was determined to have reached steady state, the cavity loss was decreased by approximately $1.1 \%$ from $0.544 \%$ to $0.538 \%$ for $2 \mu \mathrm{s}$. This had the effect of introducing a relaxation-oscillation behavior in the output of the laser. As in previous sections, only the results will be presented here. Figures 12-17 show the results of the rate equations starting from zero initial intracavity intensity flux for pump powers ranging from $225 \mathrm{~mW}$ to $500 \mathrm{~mW}$. Figures $12 \mathrm{~A}-17 \mathrm{~A}$ show both the spiking and the relaxation-oscillation behavior. Note that since the perturbation is small with respect to the steady state loss value, the response is also small and returns to the original steady state output value quicker when compared to the time it takes for the relaxation-oscillation to dampen to a steady state value at the onset of lasing (fig. 3). Figures 12B - 17B show the relaxation-oscillation response in greater detail. From these figures, the average frequency of 10 cycles was calculated. If the program was started again from the steady state population inversion and intracavity values, but this time with the cavity loss modulated at the resonant frequency, the output should be a train of spikes. This analysis, if carried out for all the different pump powers, should produce (qualitatively) similar spiking behavior. This was carried out for pumping at $350 \mathrm{~mW}$ where the cavity loss was modulated at $50.130 \mathrm{kHz}$ which is approximately $9 \%$ less than the resonant frequency determined above. The result was a train of spikes occurring at the same frequency as that of the cavity loss modulation (Figures 18-20). Figure 18 shows the laser output beginning with spiking associated with the initial turn-on followed by decay to steady state and then the onset of the spike train when the loss is modulated at a 
frequency of $50.130 \mathrm{kHz}$. Figure 19 depicts the spike train and cavity loss as a function of time. In Fig. 19, note that there is a finite delay between the onset of the loss modulation and when the output becomes a spike train with each spike having identicle peak-to-peak value. The delay occurs because, it takes time for the phase of all the circulating modes within the cavity to synchronize or lock-up. Figure 20 shows just a few of the spikes in greater detail. Again, note the delay in when the output reaches the maximum and the transition in the cavity loss from high to low value. Similar analysis was carried out at $24.009 \mathrm{kHz}$ and $11.818 \mathrm{kHz}$, corresponding to the frequencies of the first and second subharmonics, respectively, and the results are shown in figures 21-22. 

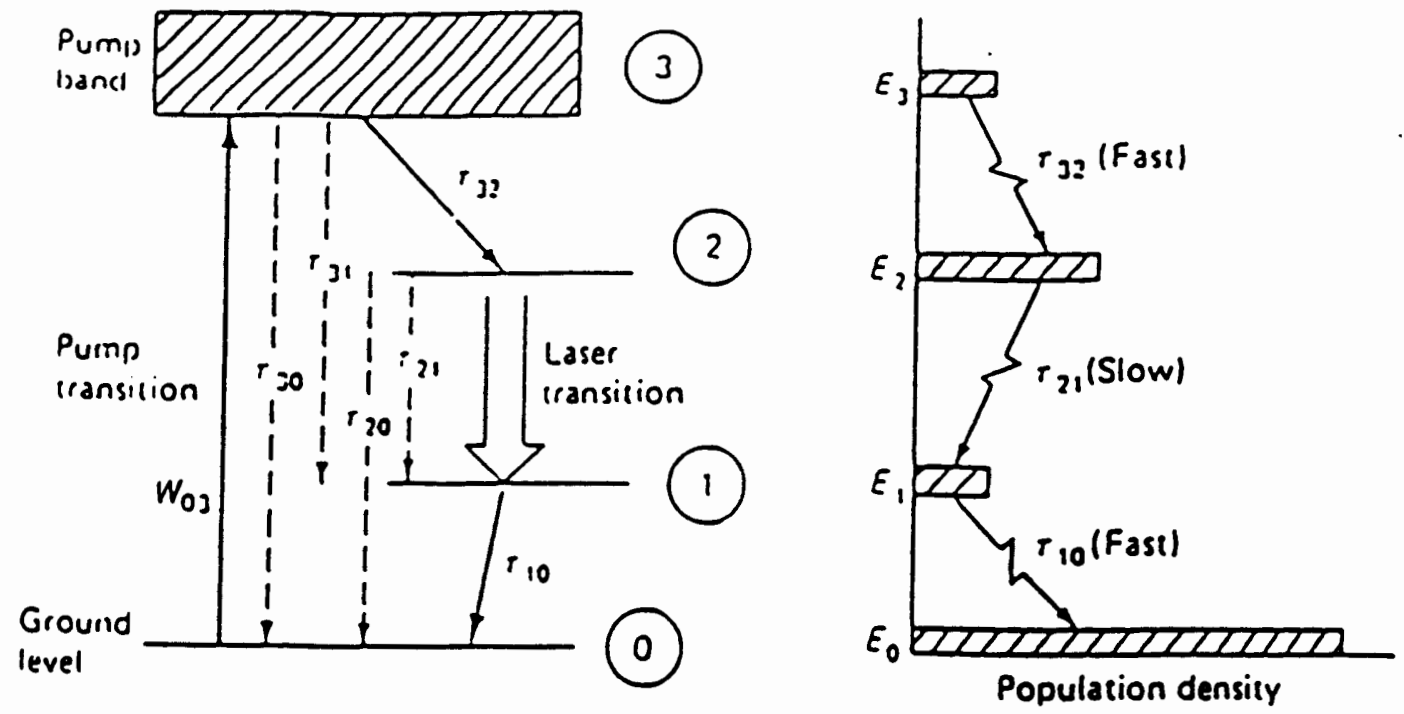

Eigure 1. Simplified energy level diagram of a four level laser [5].

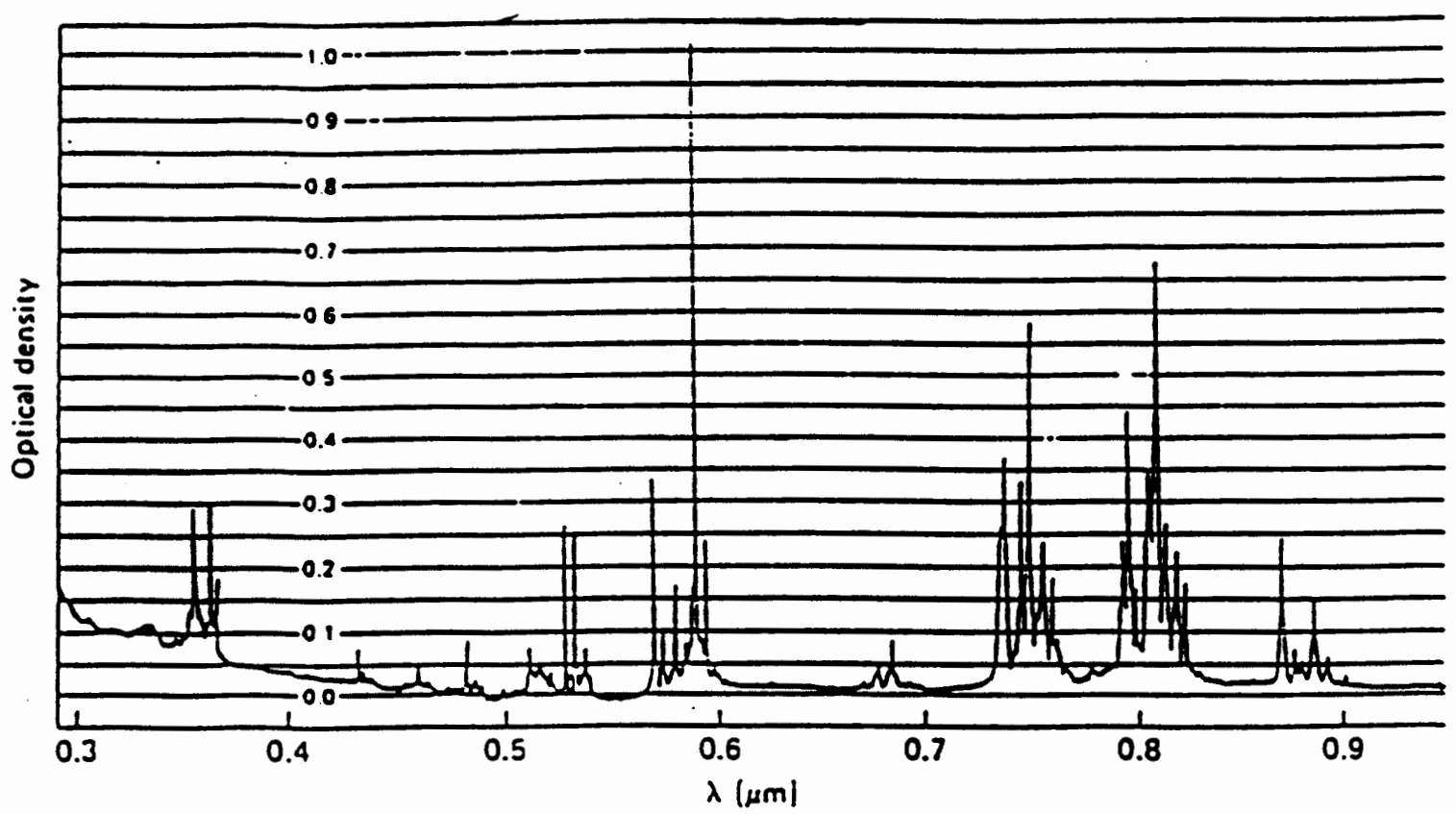

Eigure 2. Absorption spectrum of Nd:YAG at $300 \mathrm{~K}$ [5]. 


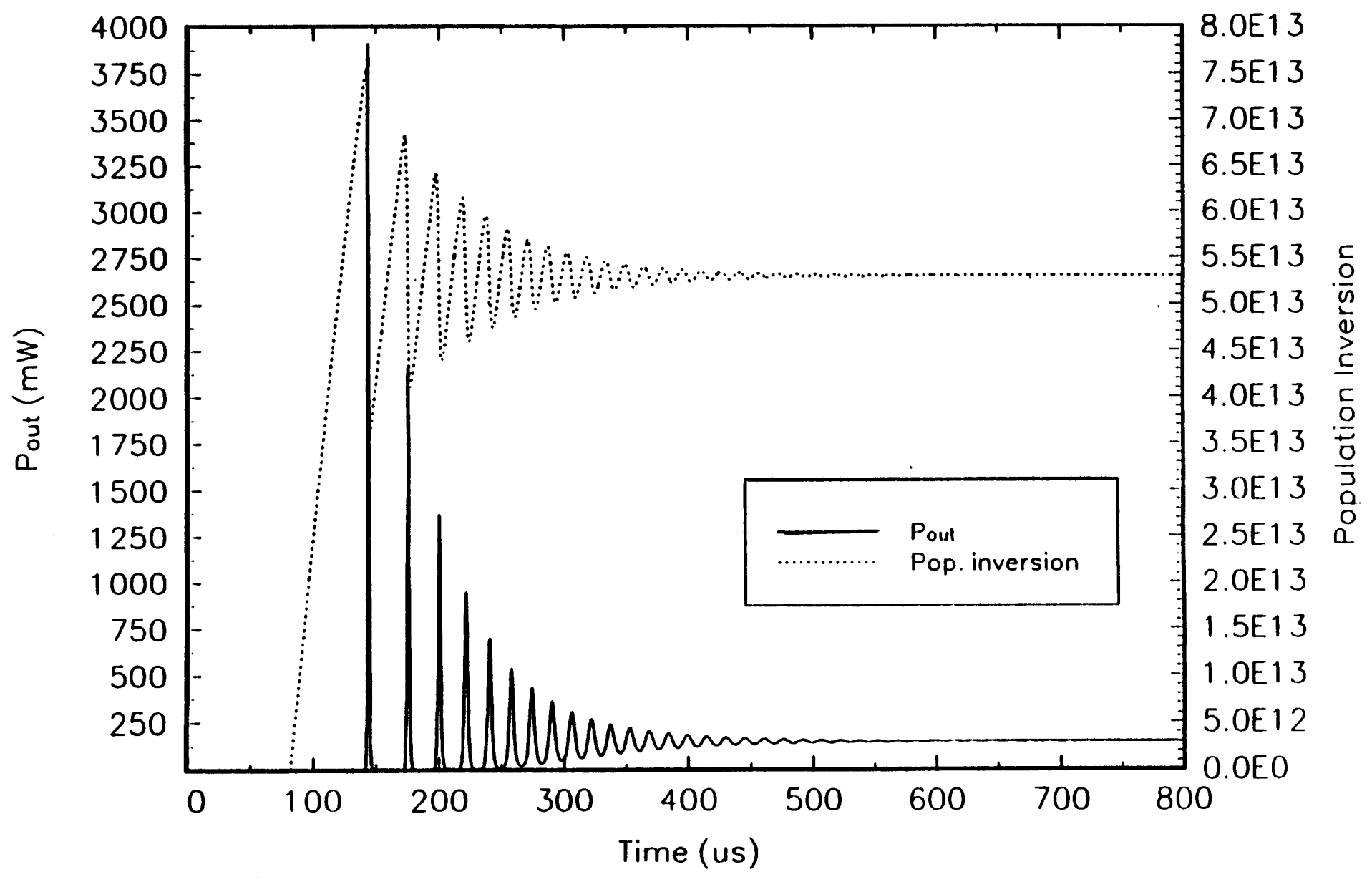

Eigure 3. Computer simulation of the spiking behavior in Nd:YAG laser. 


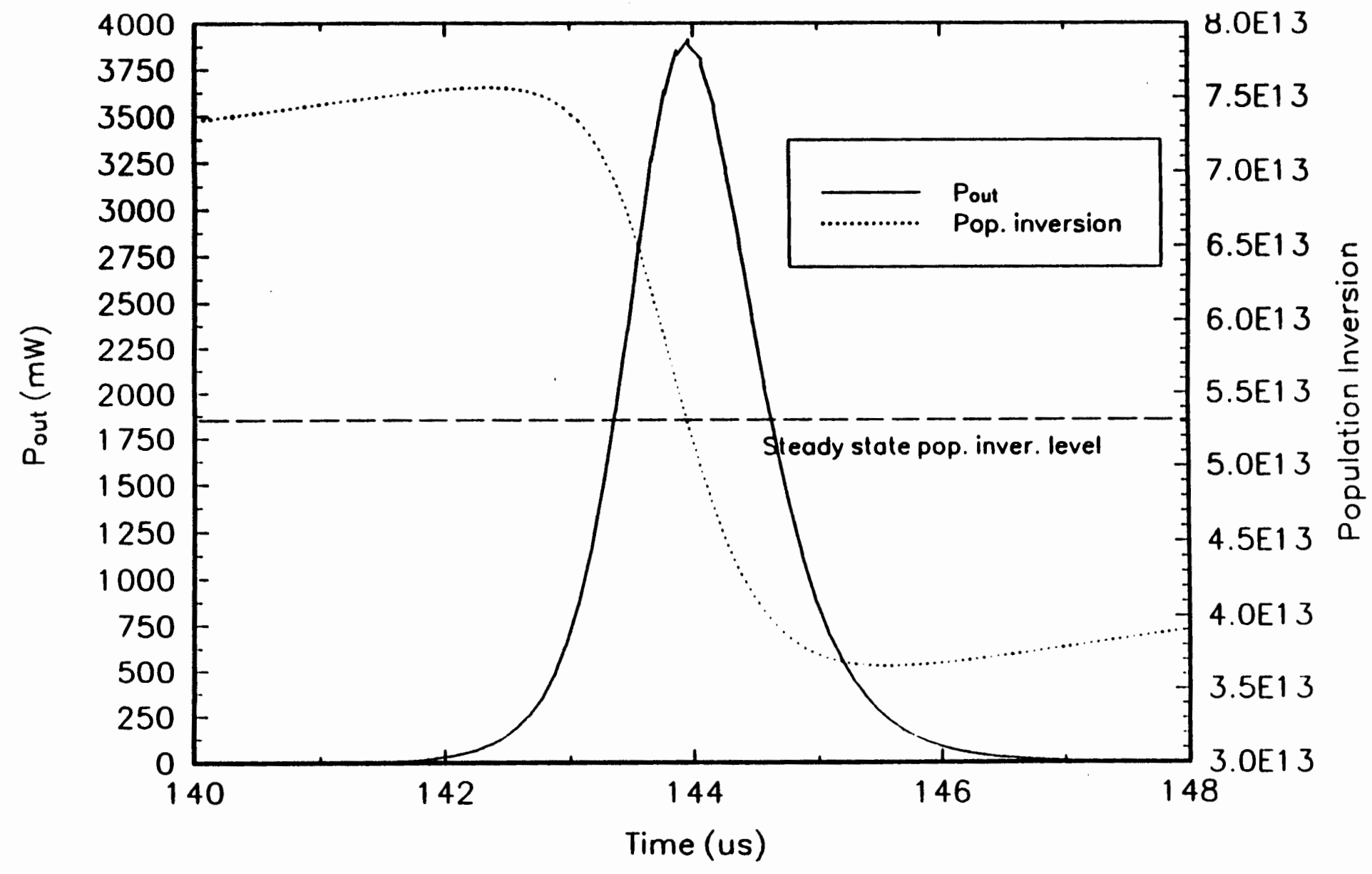

Eigure 4. Evolution of a spike. 


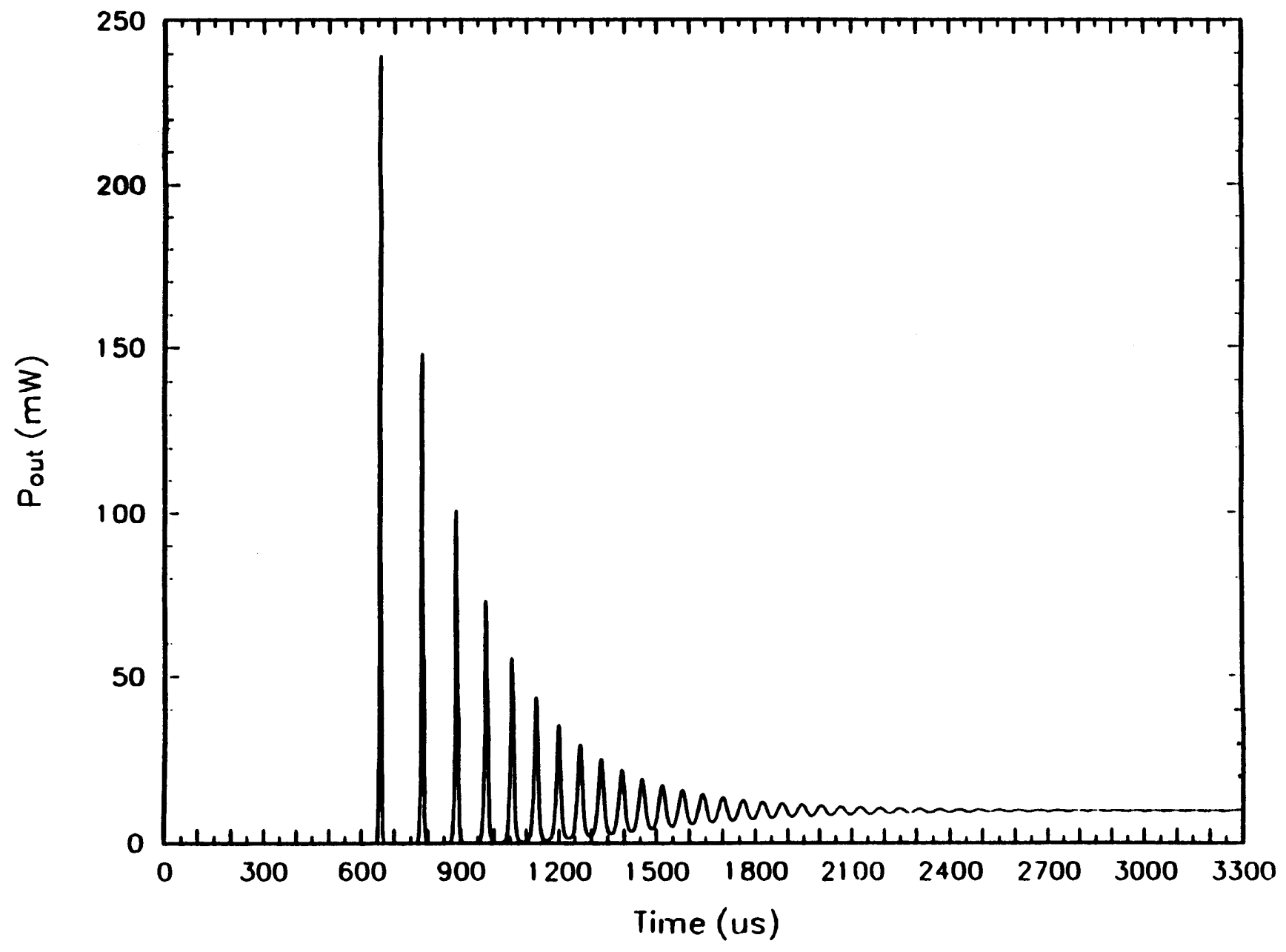

Eigure 5. Spiking behavior in Nd:YAG laser, $P_{\text {in }}=225 \mathrm{~mW}$. 


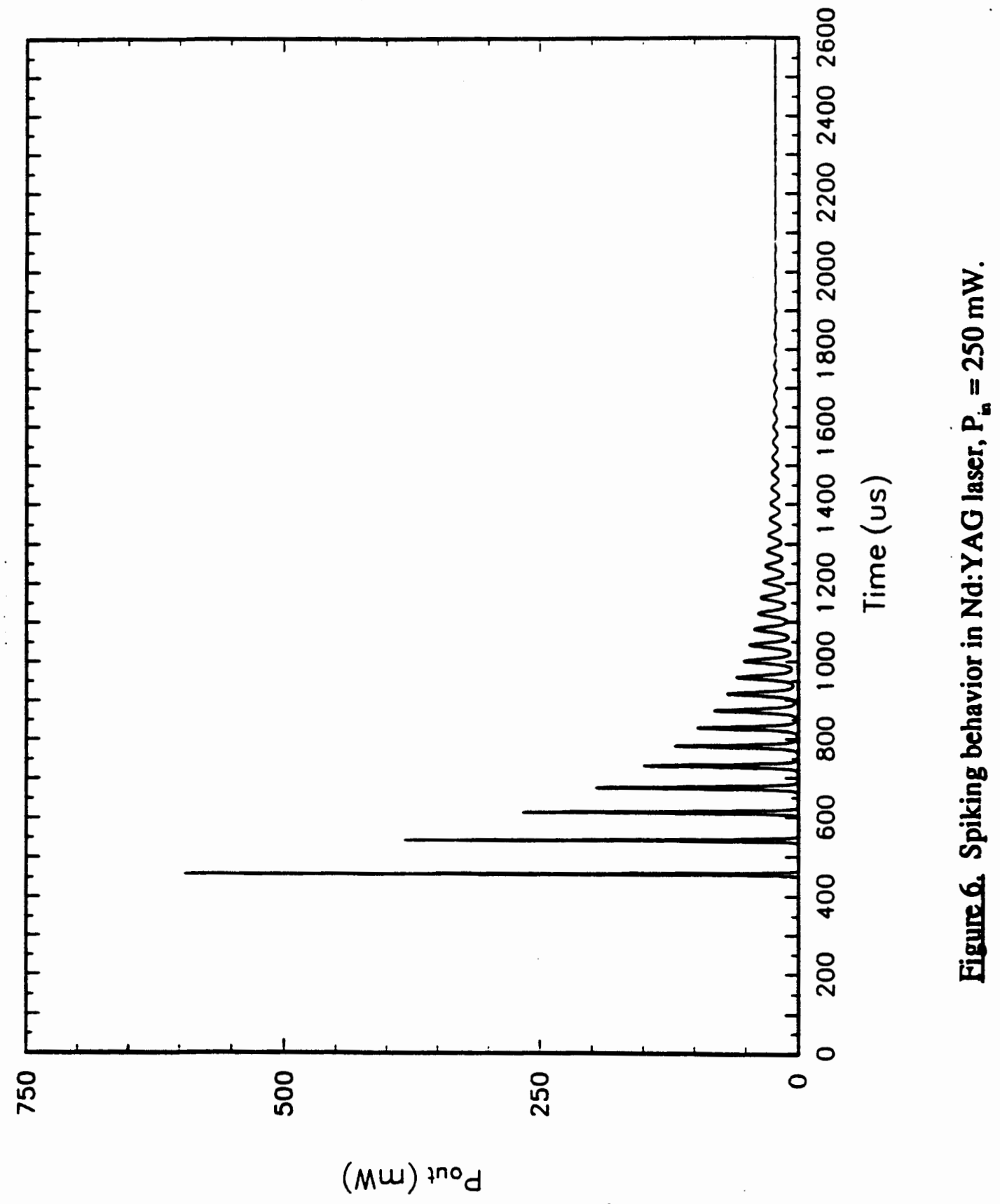




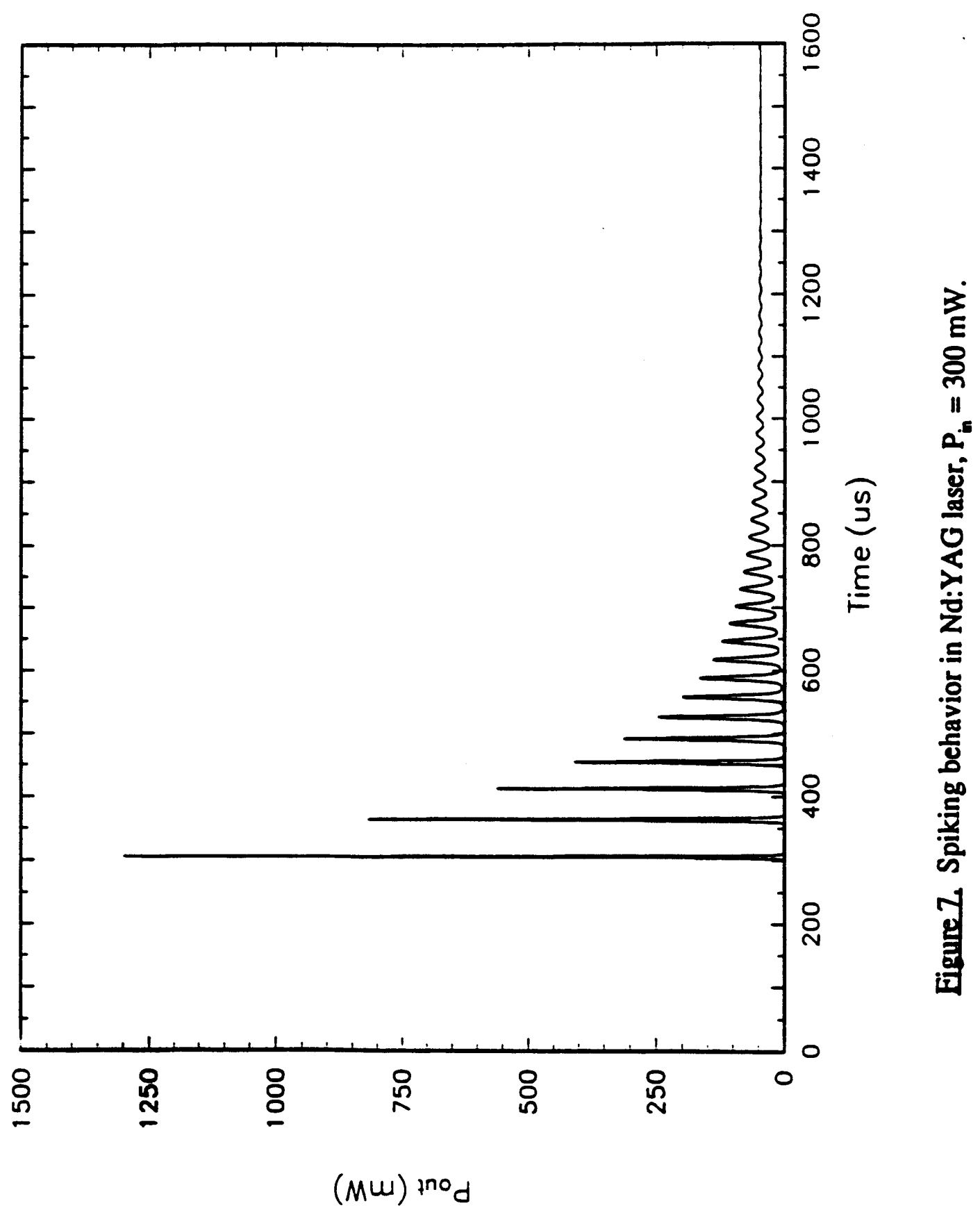




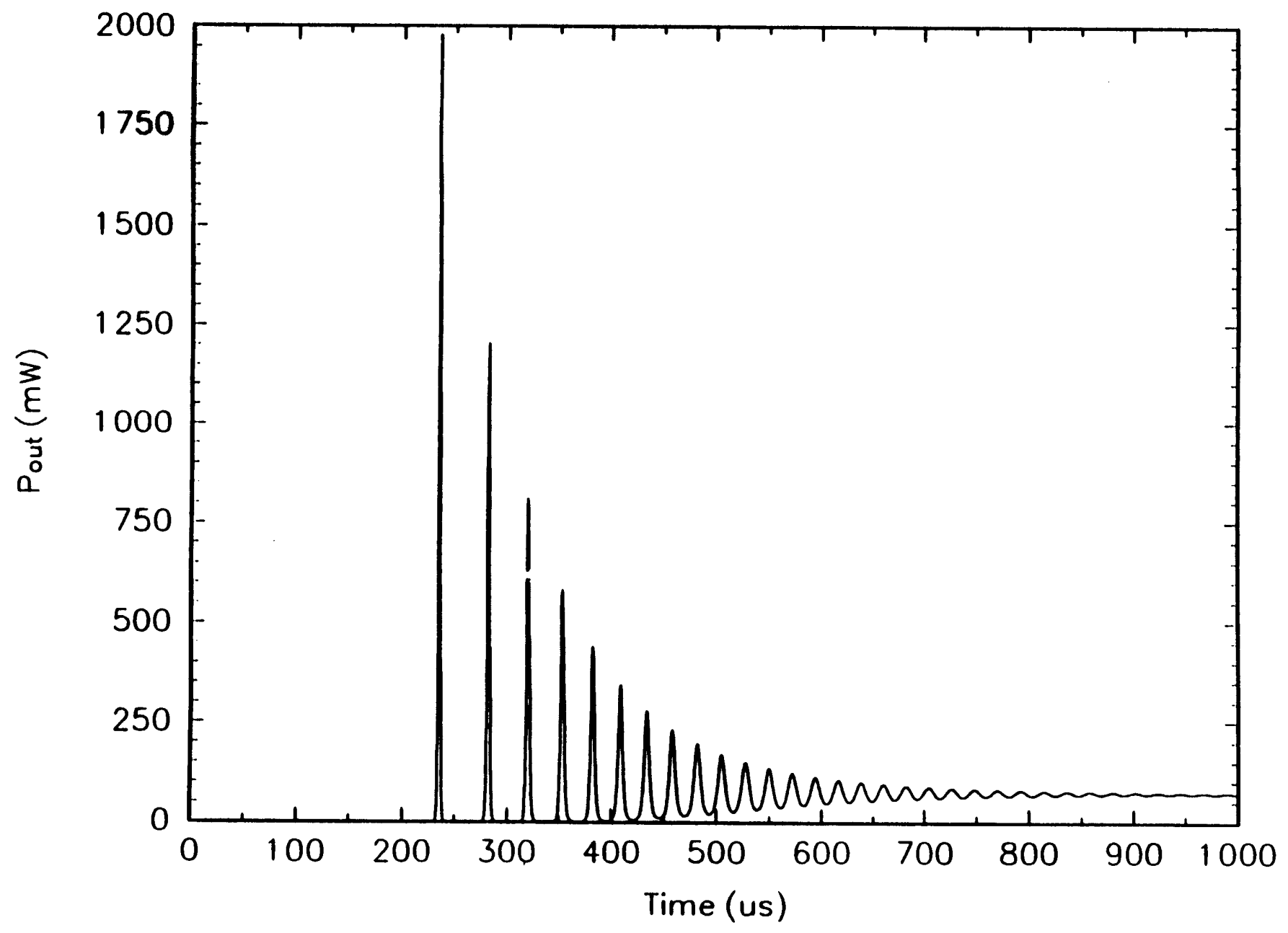

Eigure 8. Spiking behavior in Nd:YAG laser, $P_{\text {in }}=350 \mathrm{~mW}$. 


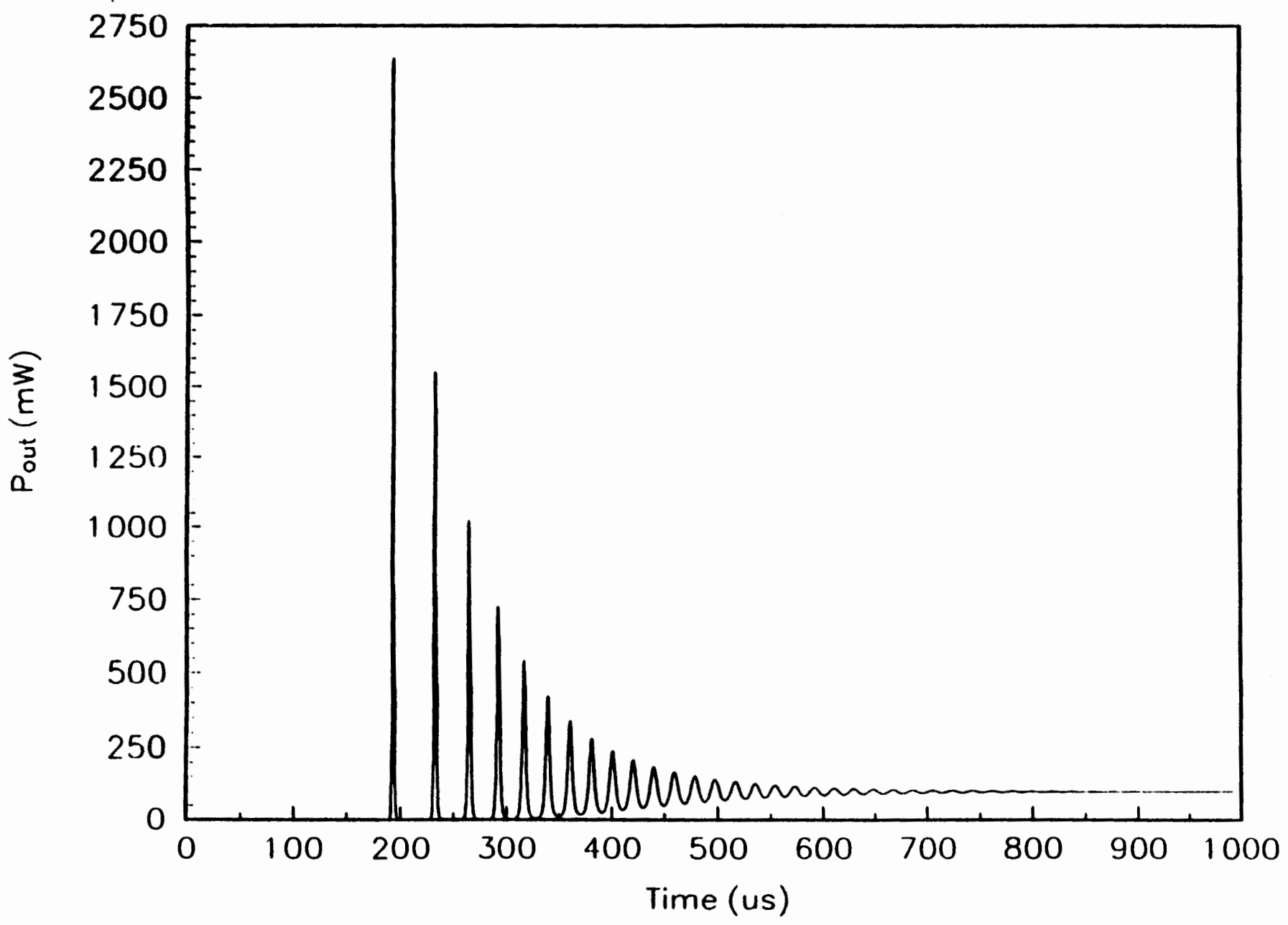

Eigure 2. Spiking behavior in Nd:YAG laser, $P_{i n}=400 \mathrm{~mW}$. 


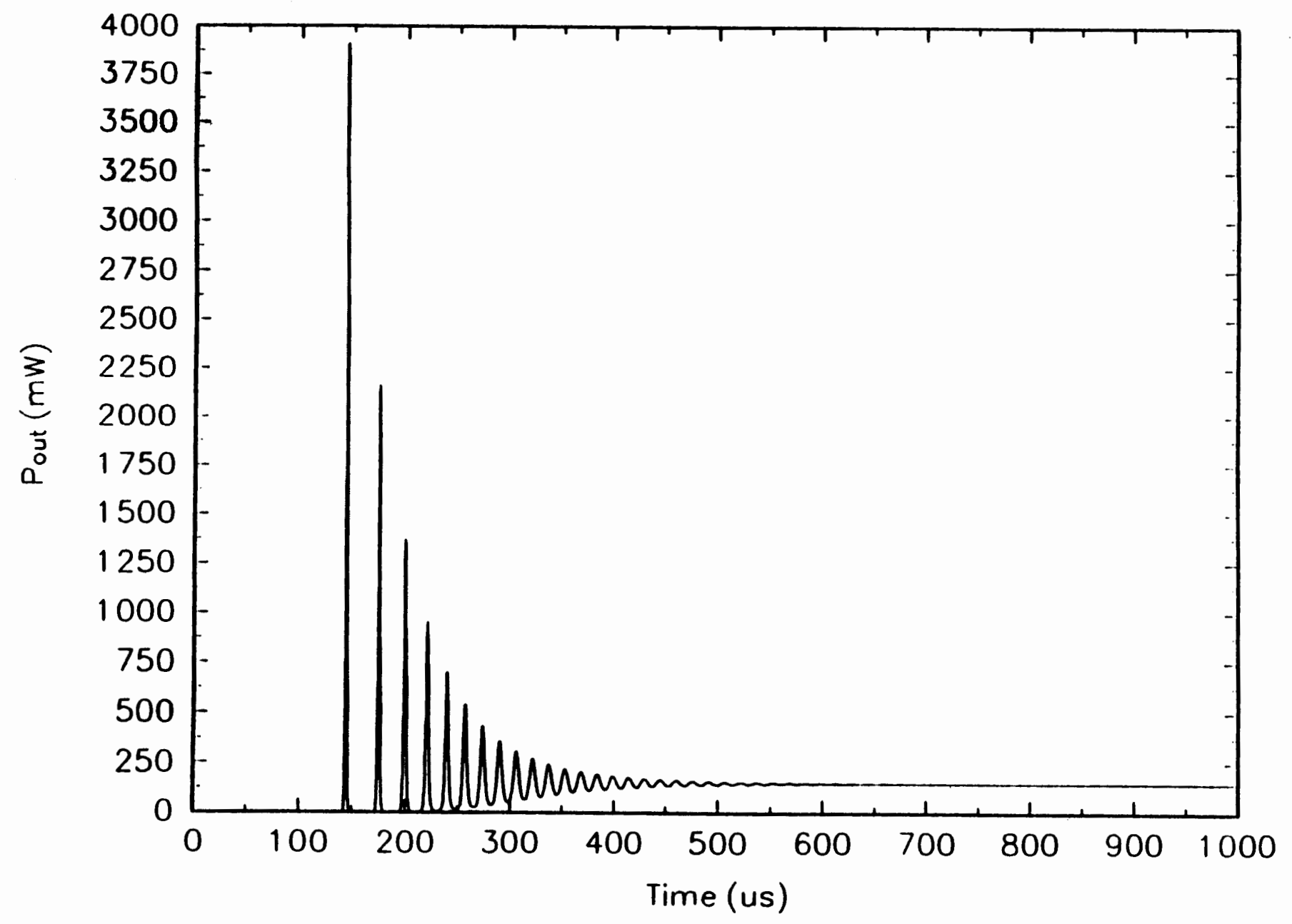

Eigure 10. Spiking behavior in Nd:YAG laser, $P_{\text {in }}=500 \mathrm{~mW}$. 


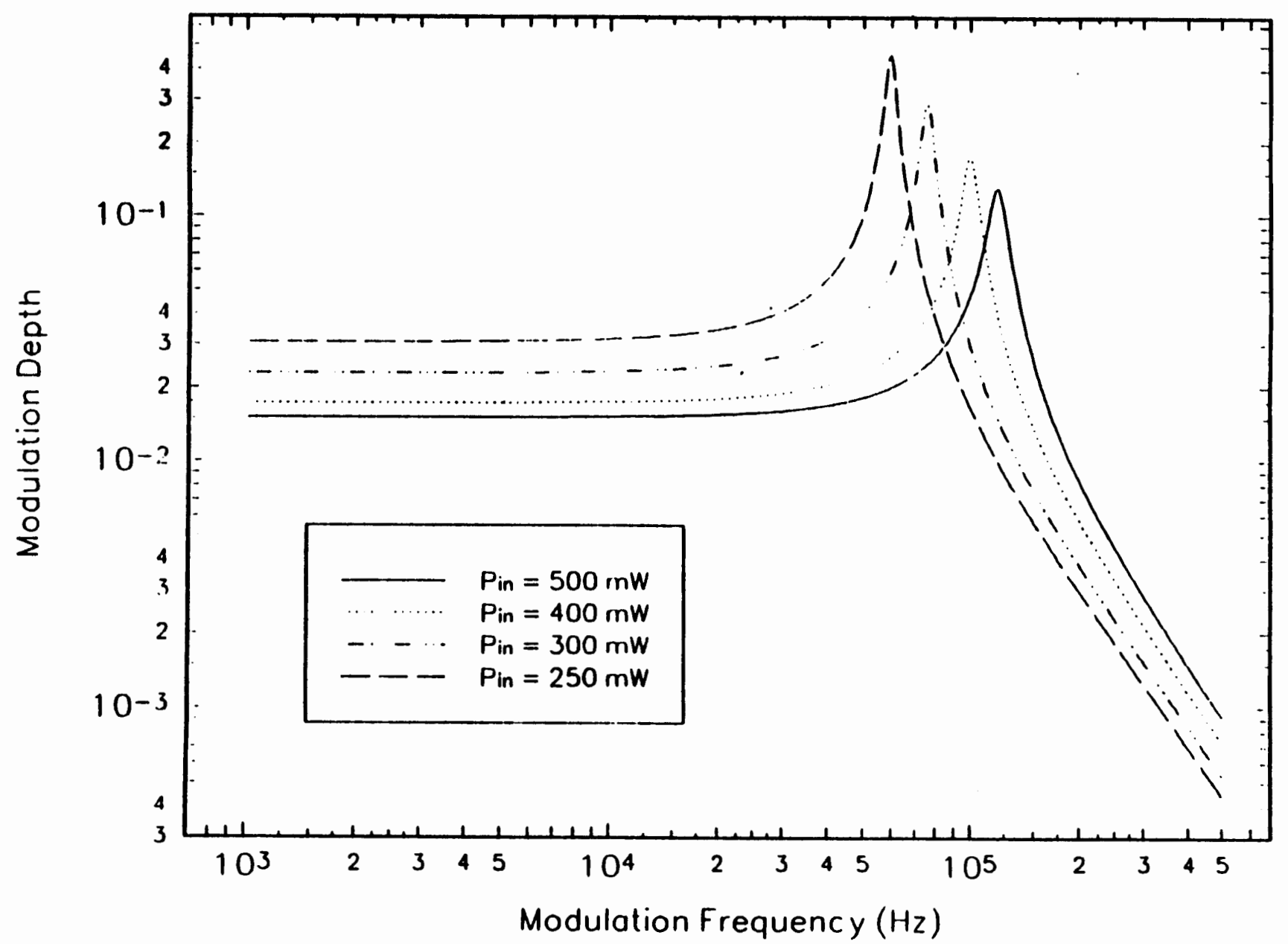

Eigure 11 Change in modulation depth and the resonant frequency as function of $P_{\text {in }}$. 


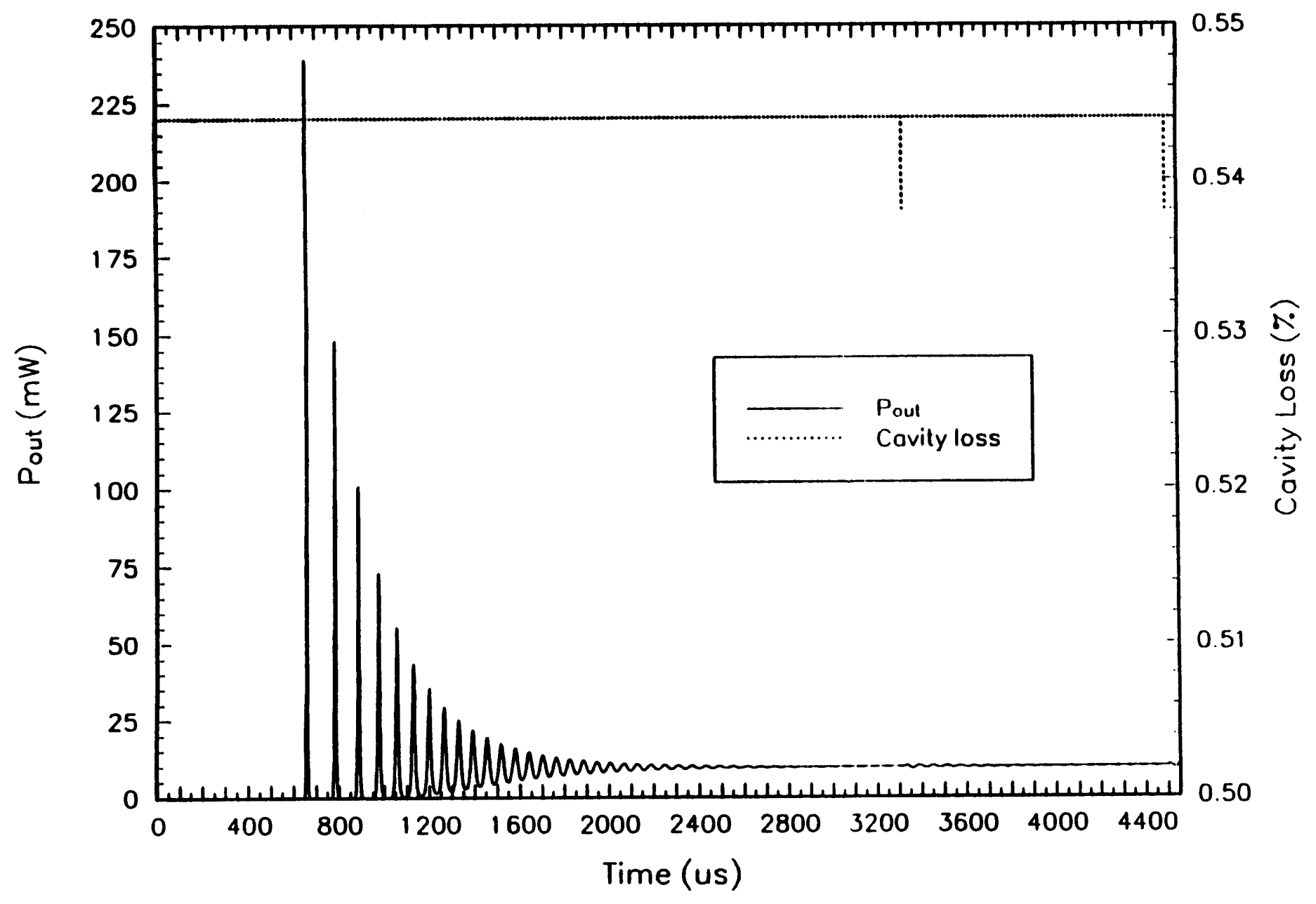

Figure 12A. Spiking and relaxation oscillation behavior is Nd:YAG laser, $P_{i n}=225 \mathrm{~mW}$. 


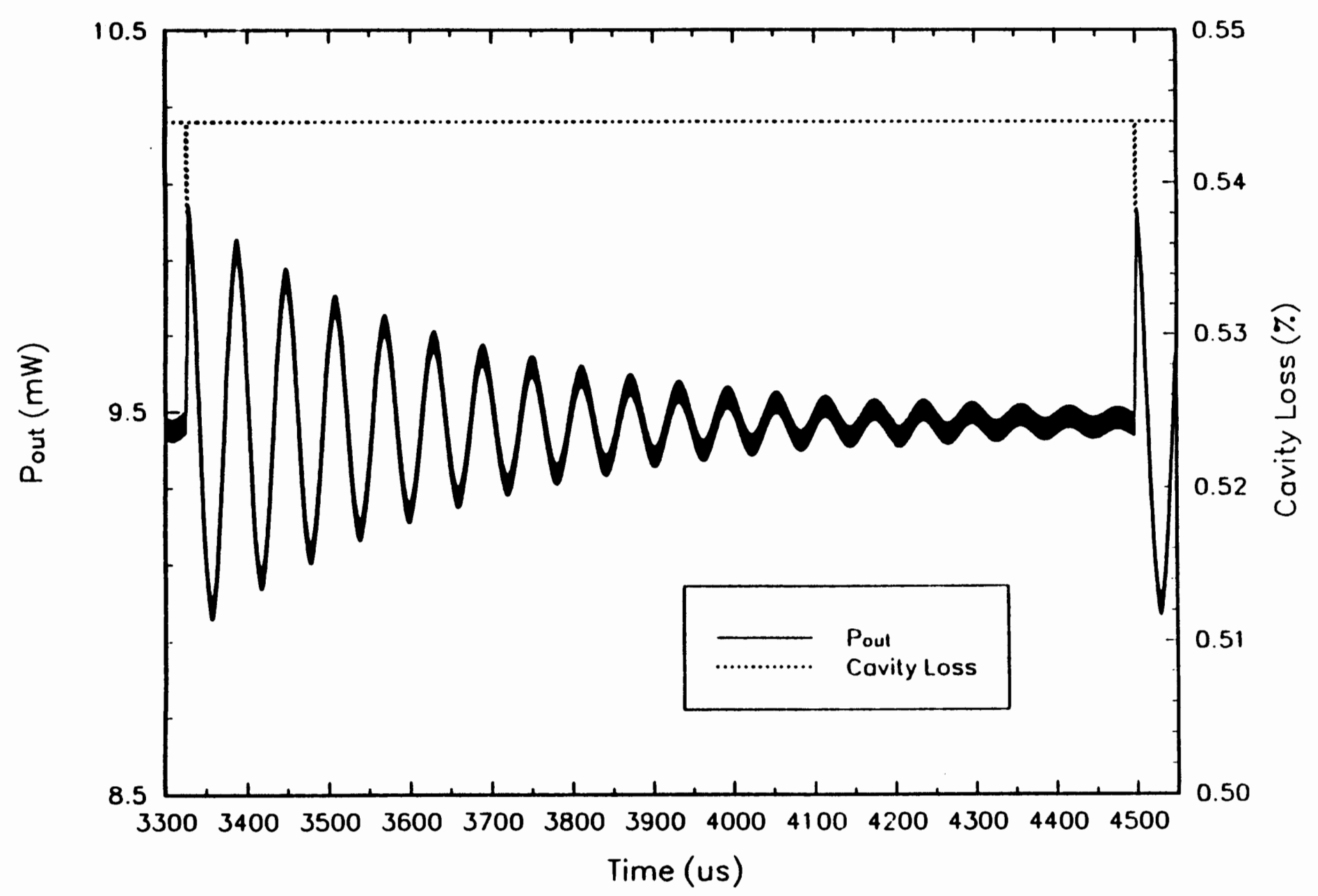

Eigure 12B. Details of the relaxation oscillations at $f_{R-0}=16.485 \mathrm{kHz}$. 


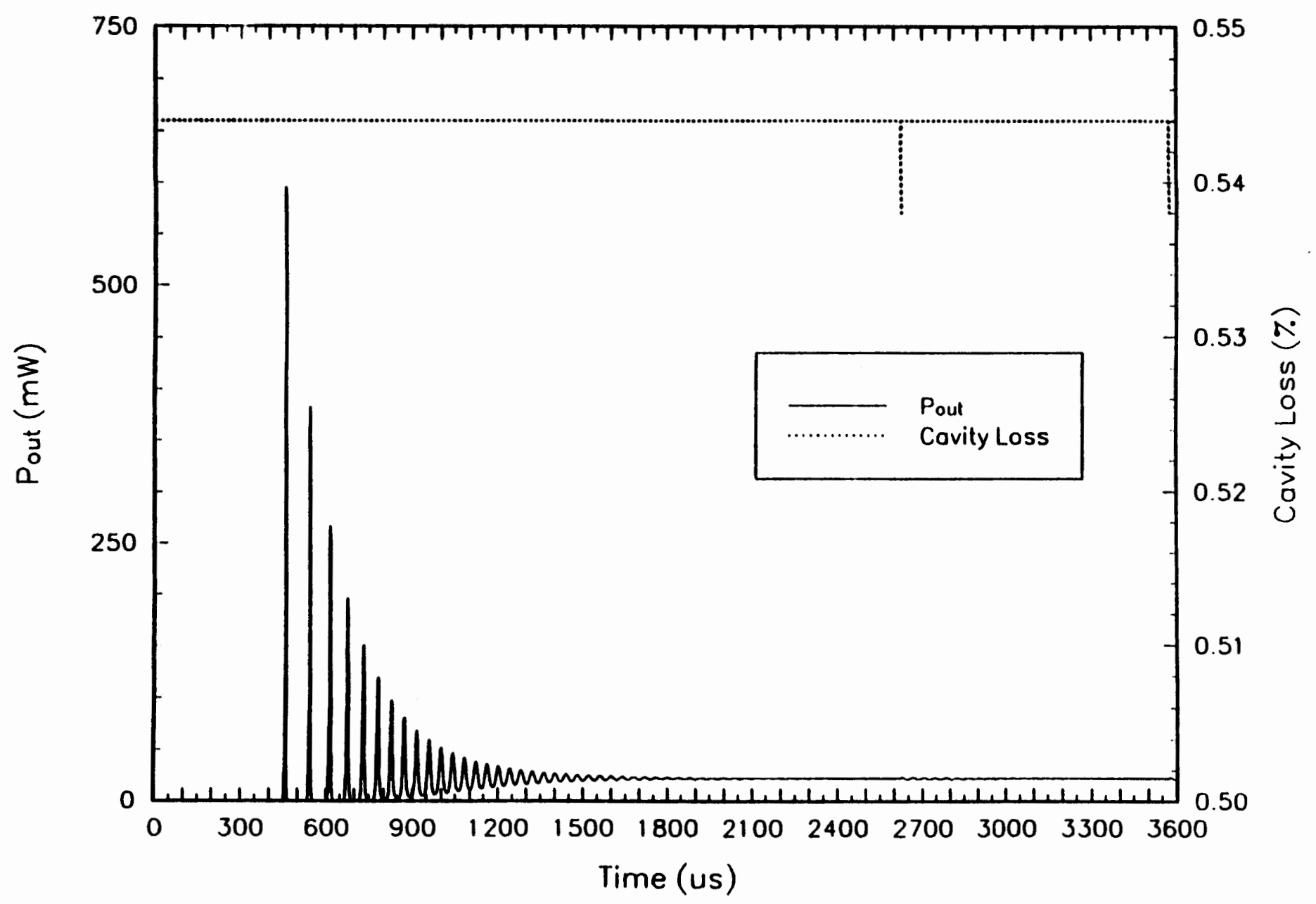

Figure 13A. Spiking and relaxation oscillation behavior in Nd:YAG laser, $P_{i n}=250 \mathrm{~mW}$. 


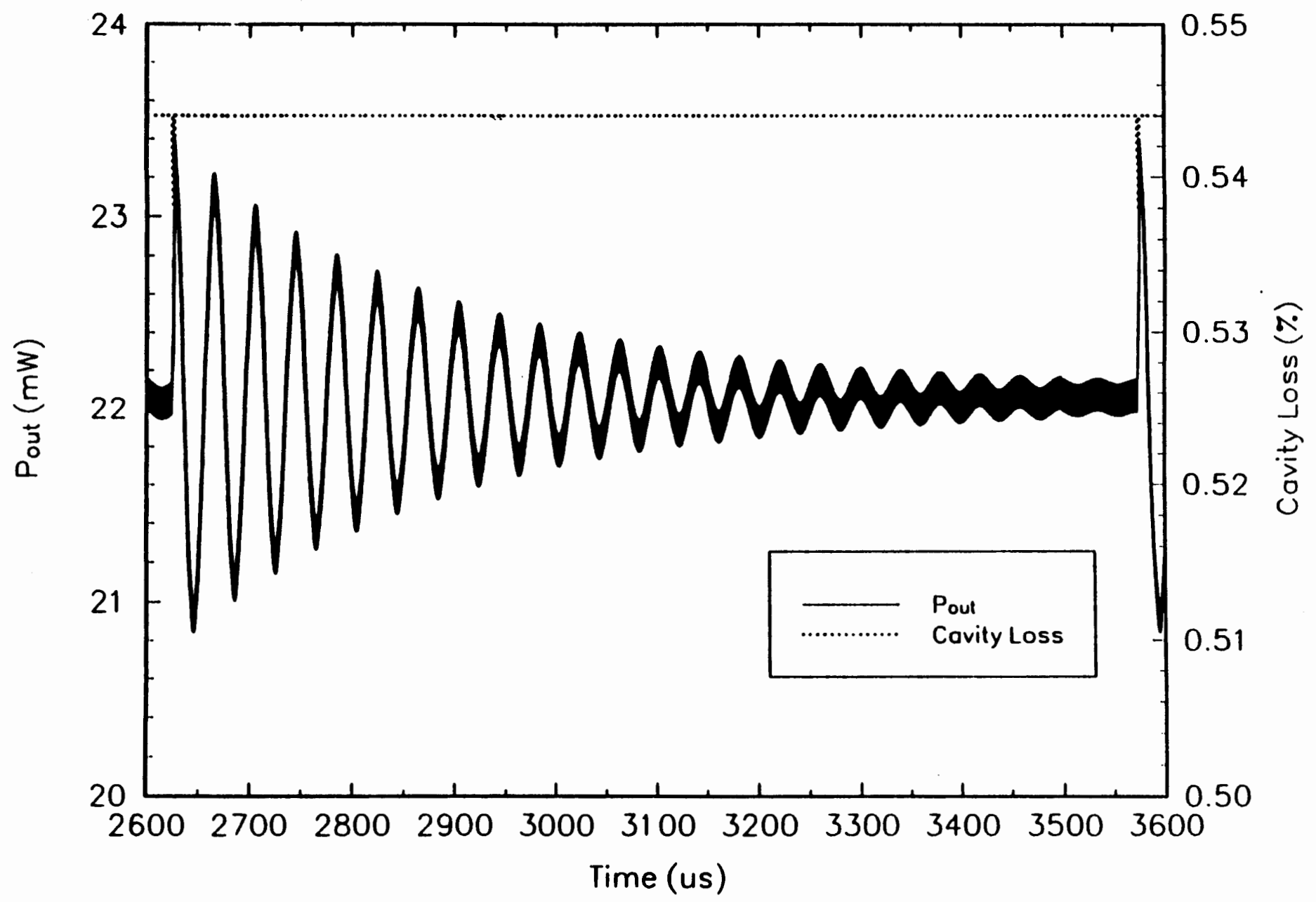

Eigure 13B. Details of the relaxation oscillations at $f_{R-0}=25.187 \mathbf{k H z}$. 


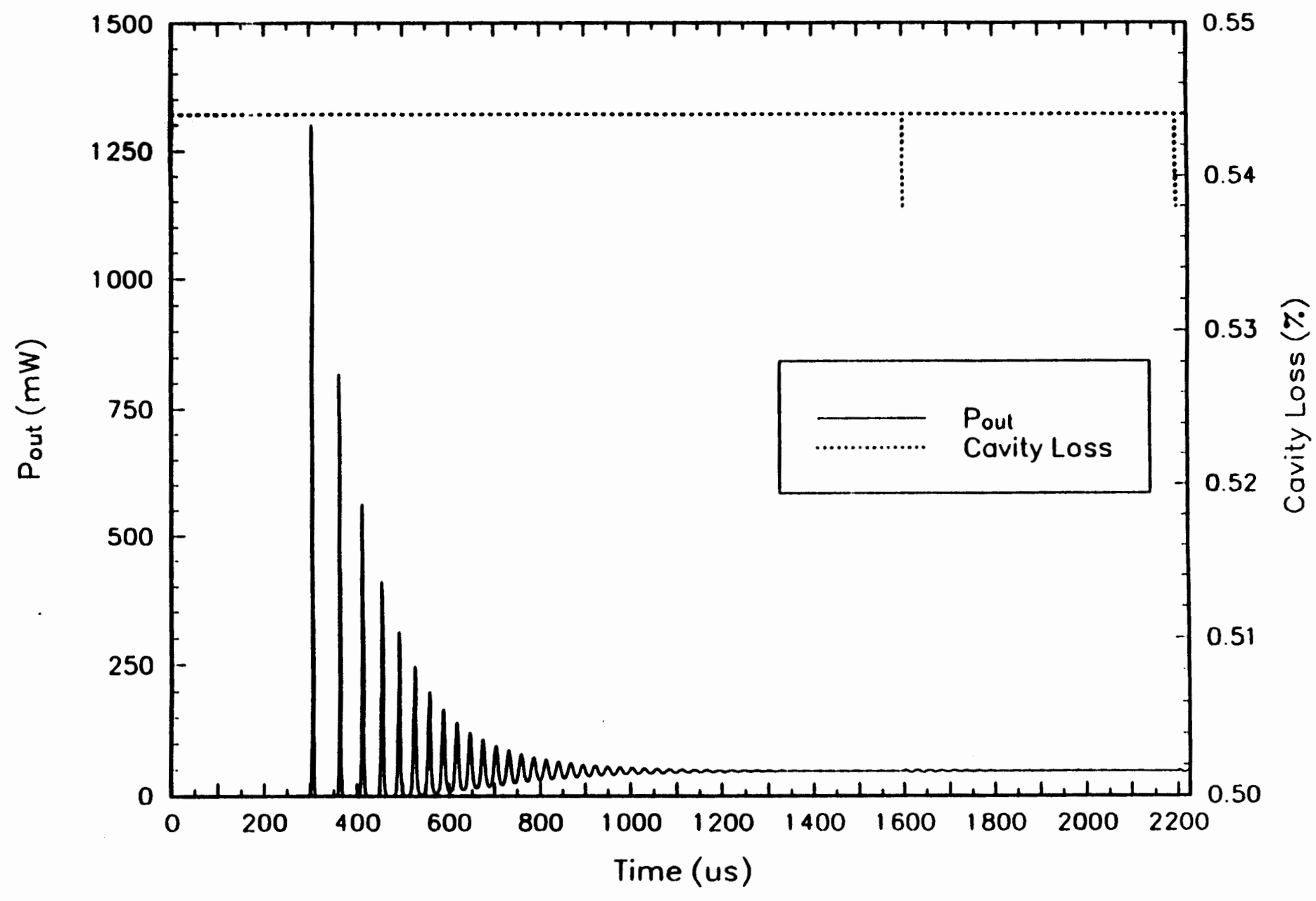

Eigure 14A. Spiking and relaxation oscillation behavior in Nd:YAG laser, $P_{\mathrm{h}}=300 \mathrm{~mW}$. 


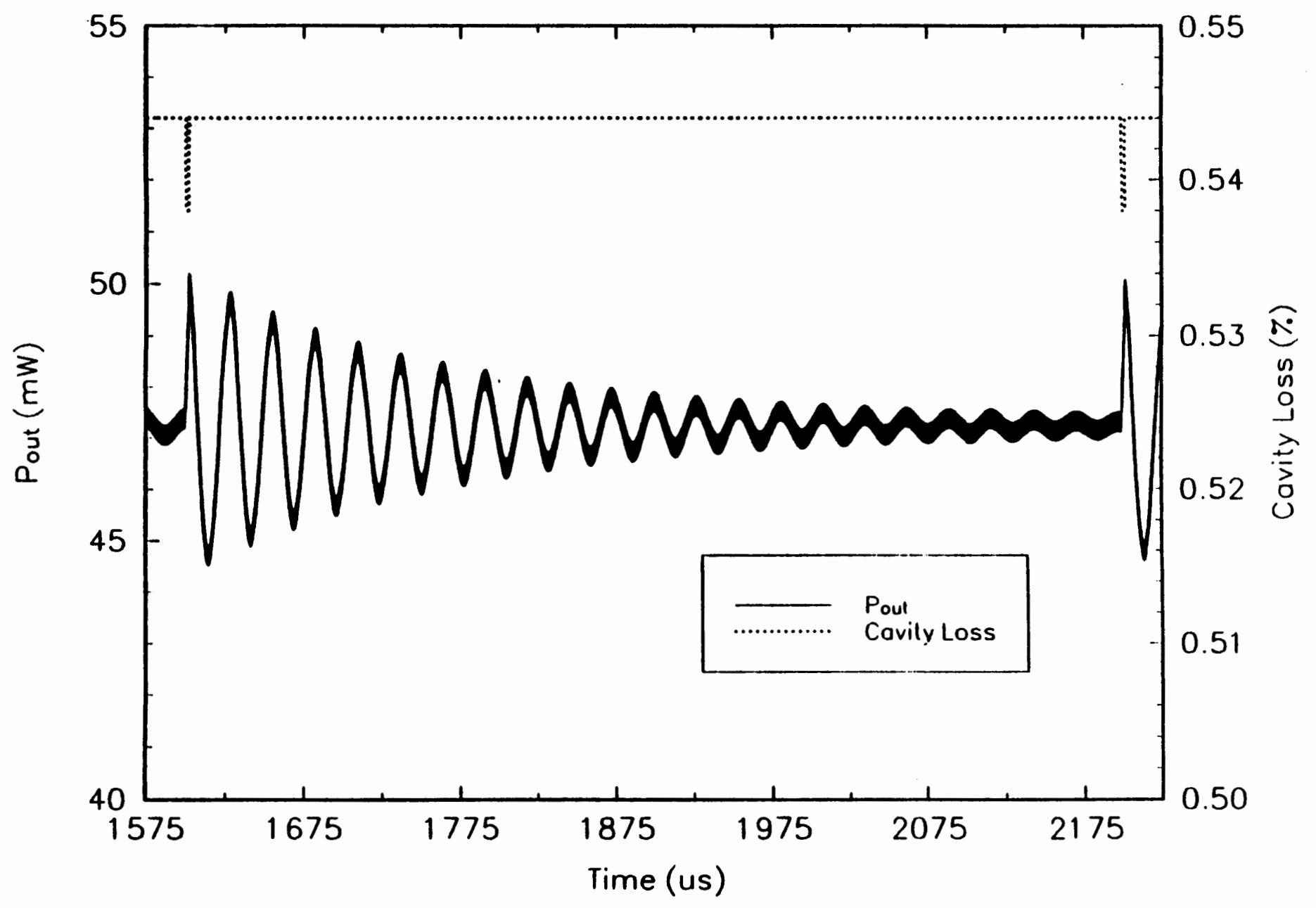

Eigure 14B. Details of the relaxation oscillations at $f_{R-0}=36.874 \mathrm{kHz}$. 


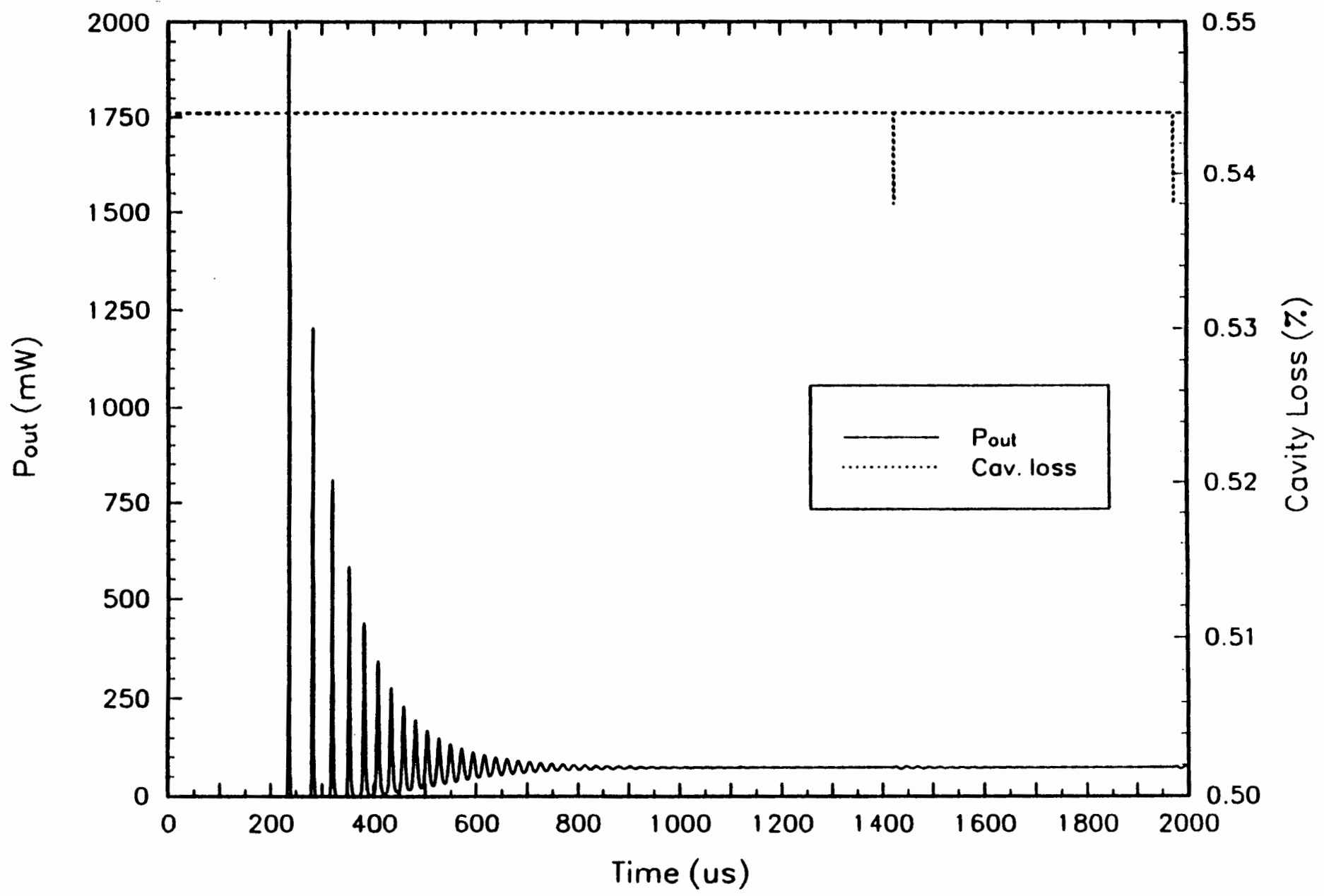

Eigure 15A. Spiking and relaxation oscillation behavior in Nd:YAG laser, $\ddot{P}_{\text {in }}=350 \mathrm{~mW}$. 


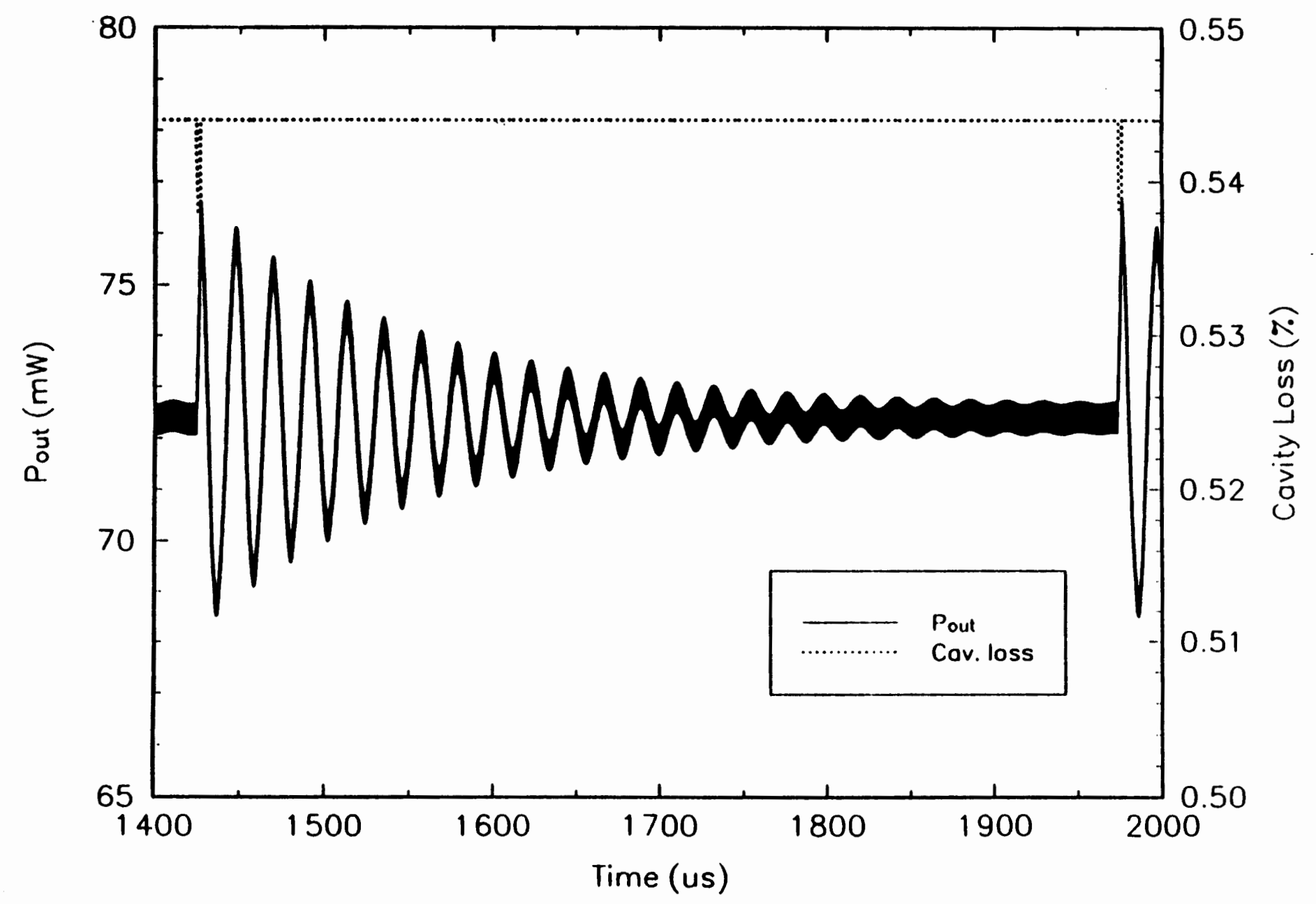

Figure 15B. Details of the relaxation oscillations at $f_{R-0}=45.618 \mathrm{kHz}$. 


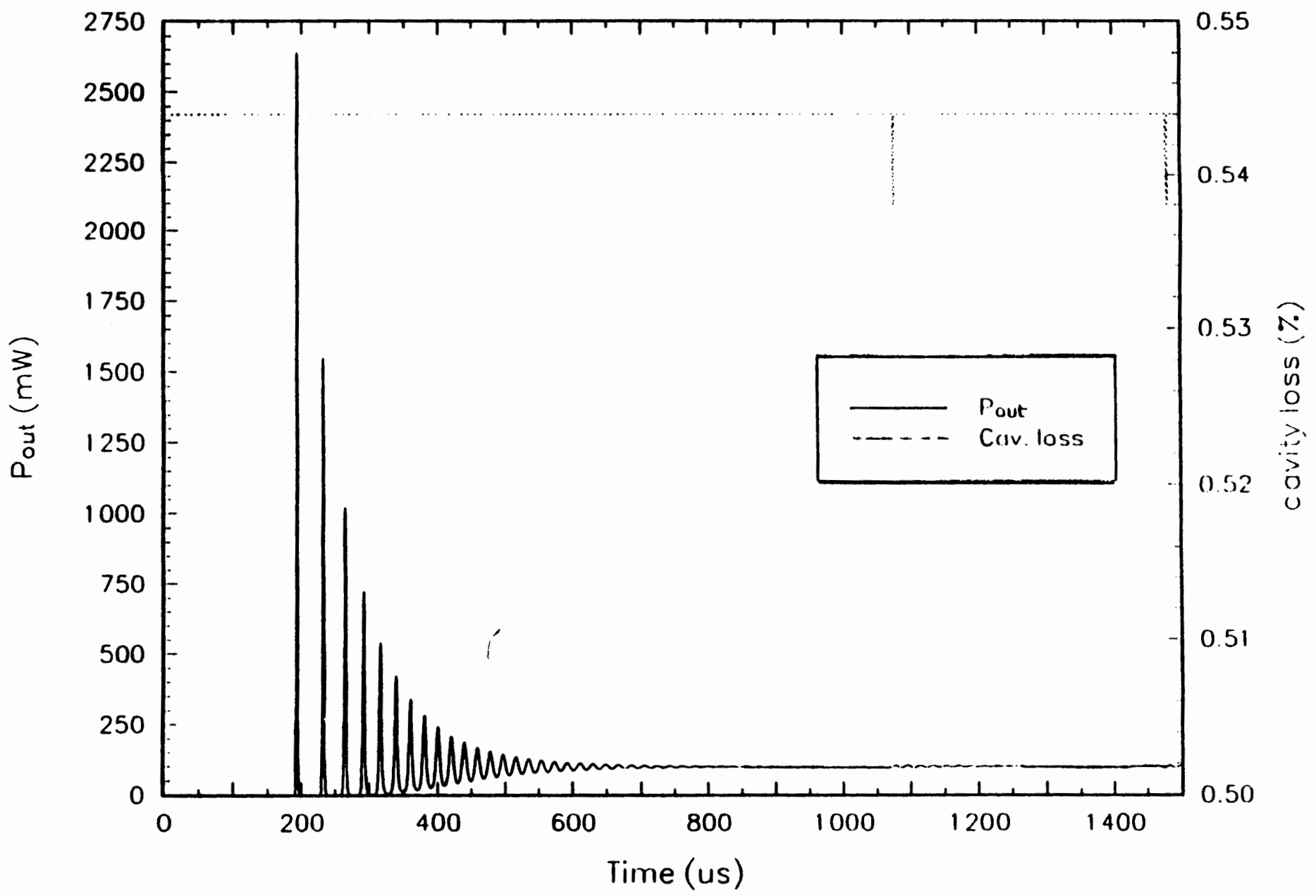

Eigure 16A. Spiking and relaxation oscillation behavior in Nd:YAG laser, $P_{\text {in }}=400 \mathrm{~mW}$. 


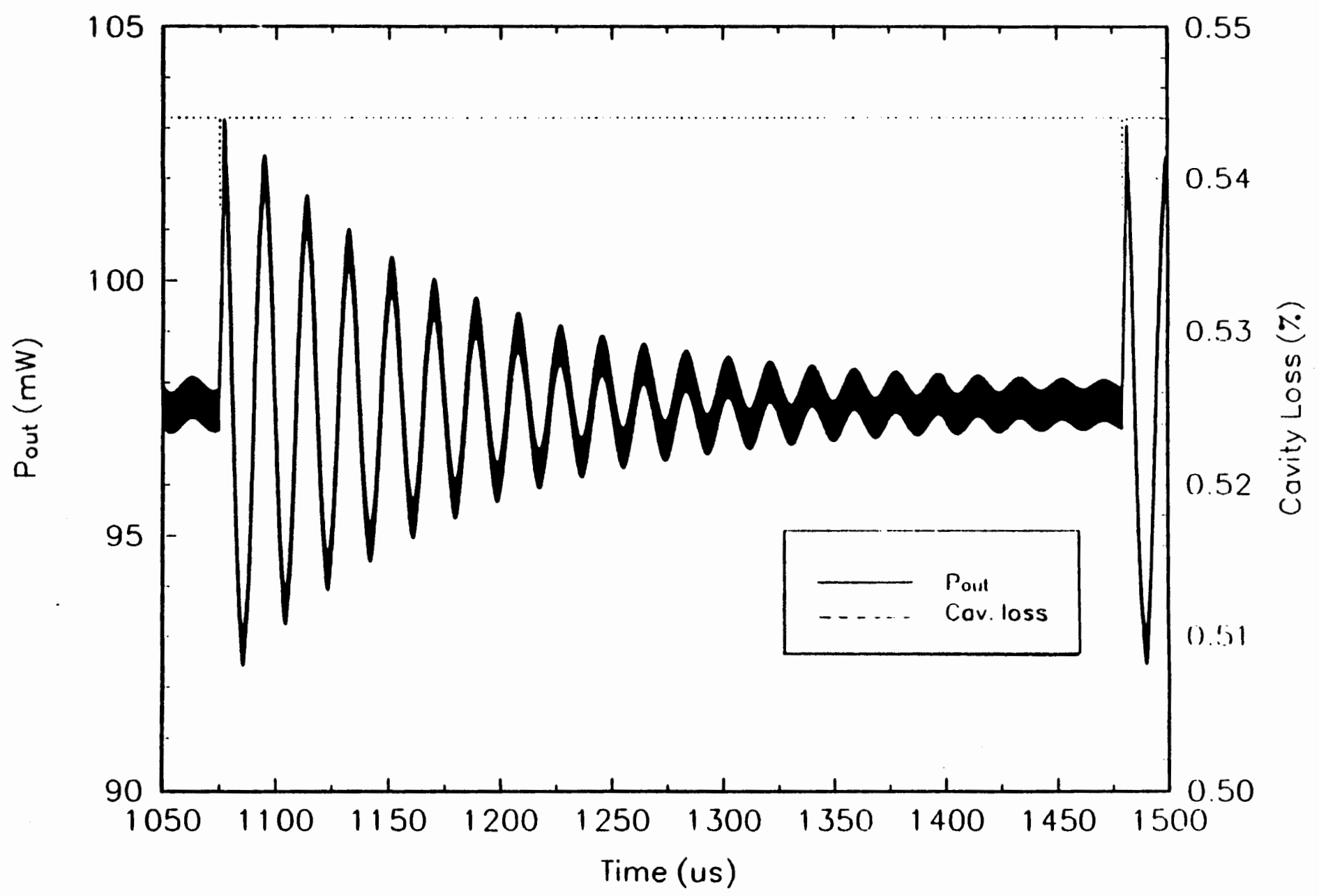

Figure 16B. Details of the relaxation oscillations at $f_{R \cdot 0}=52.946 \mathrm{kHz}$. 


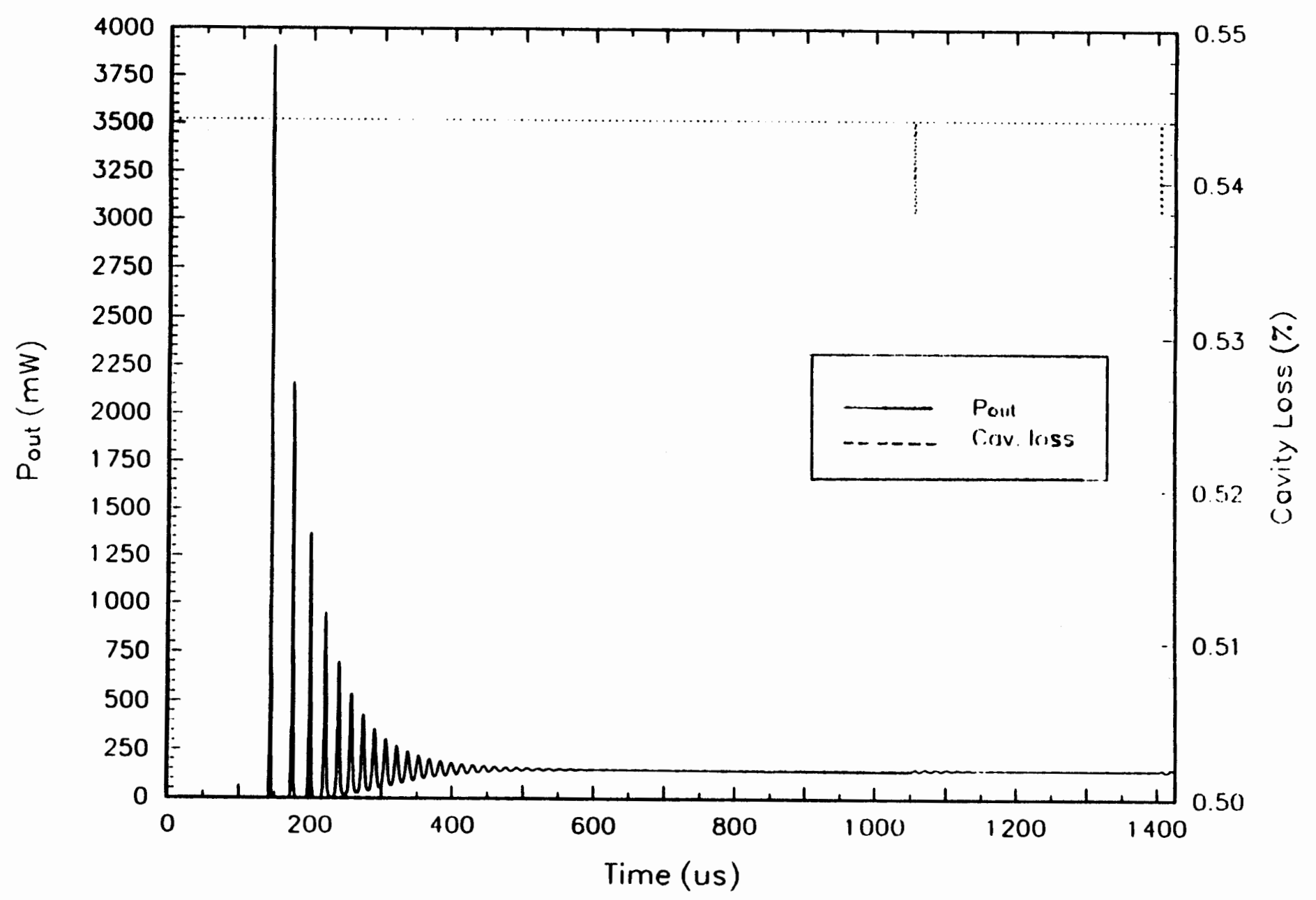

Eigure 17A. Spiking and relaxation oscillation behavior in Nd:YAG laser, $P_{\text {in }}=500 \mathrm{~mW}$. 


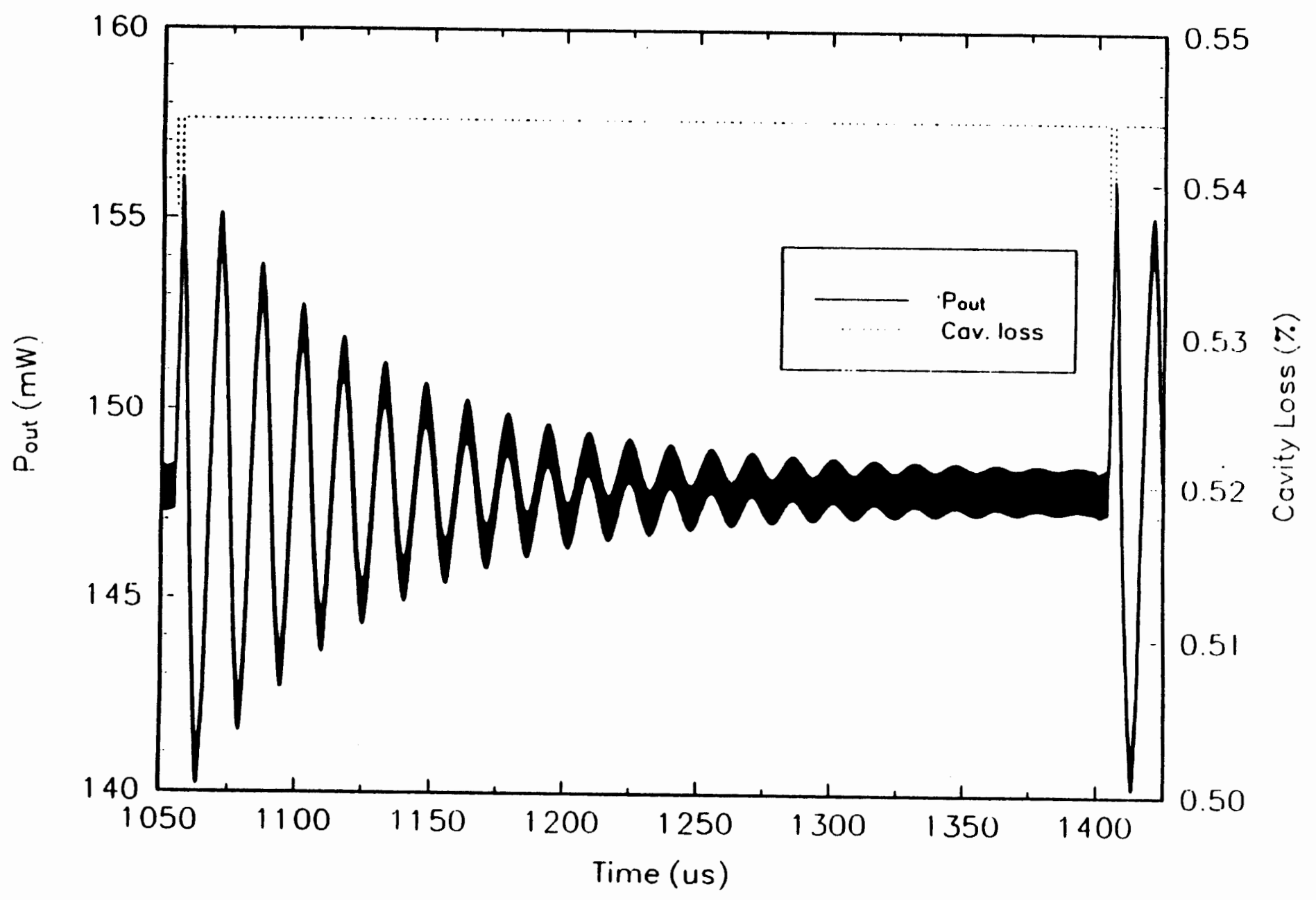

Eigure 17B. Details of the relaxation oscillations at $f_{R-0}=65.229 \mathrm{kHz}$. 


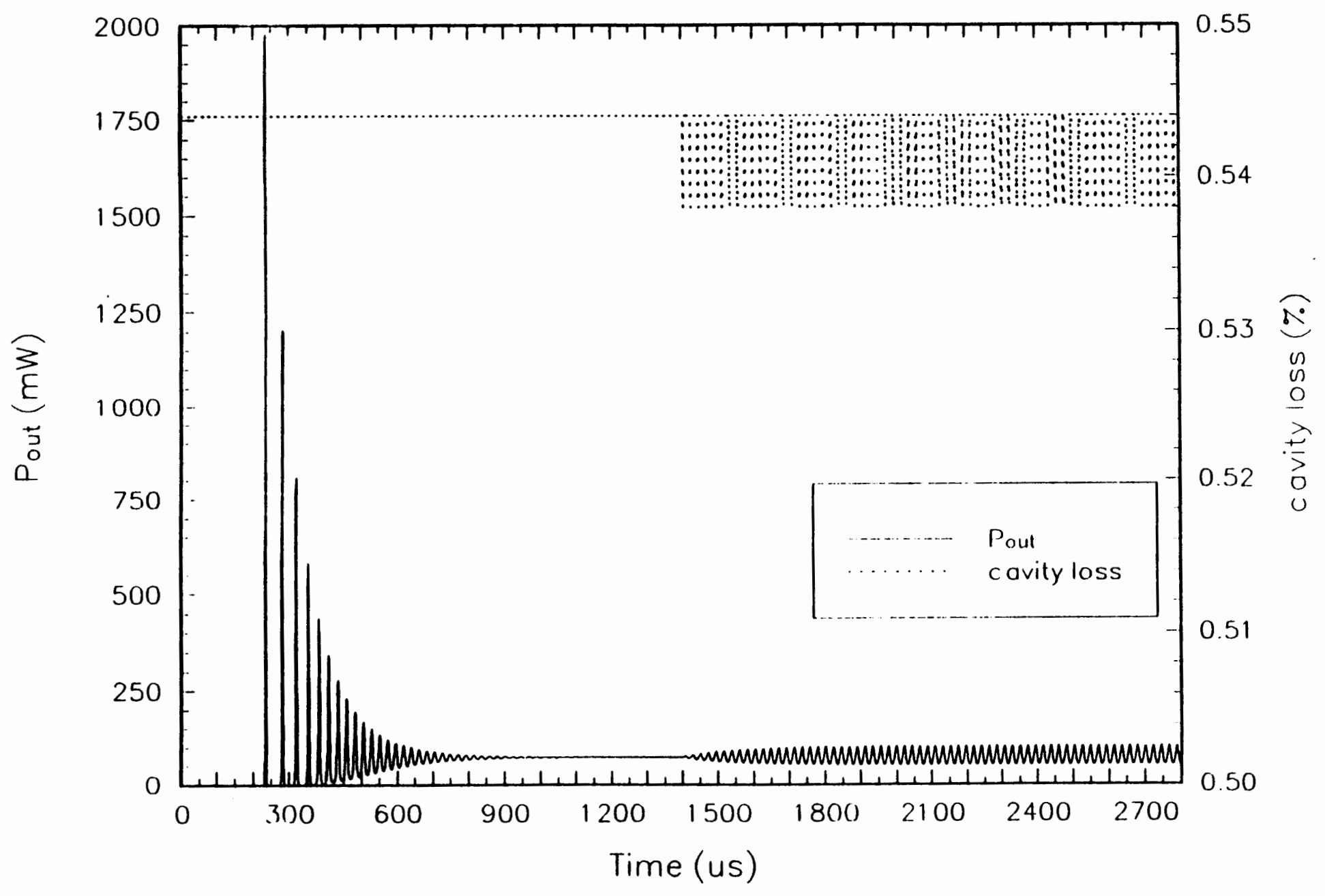

Figure 18. Spiking and giant pulsing in Nd:YAG laser, $P_{\text {in }}=350 \mathrm{~mW}$, loss modulation frequency is $f_{\text {mod }}=50.130 \mathrm{kHz}$. 


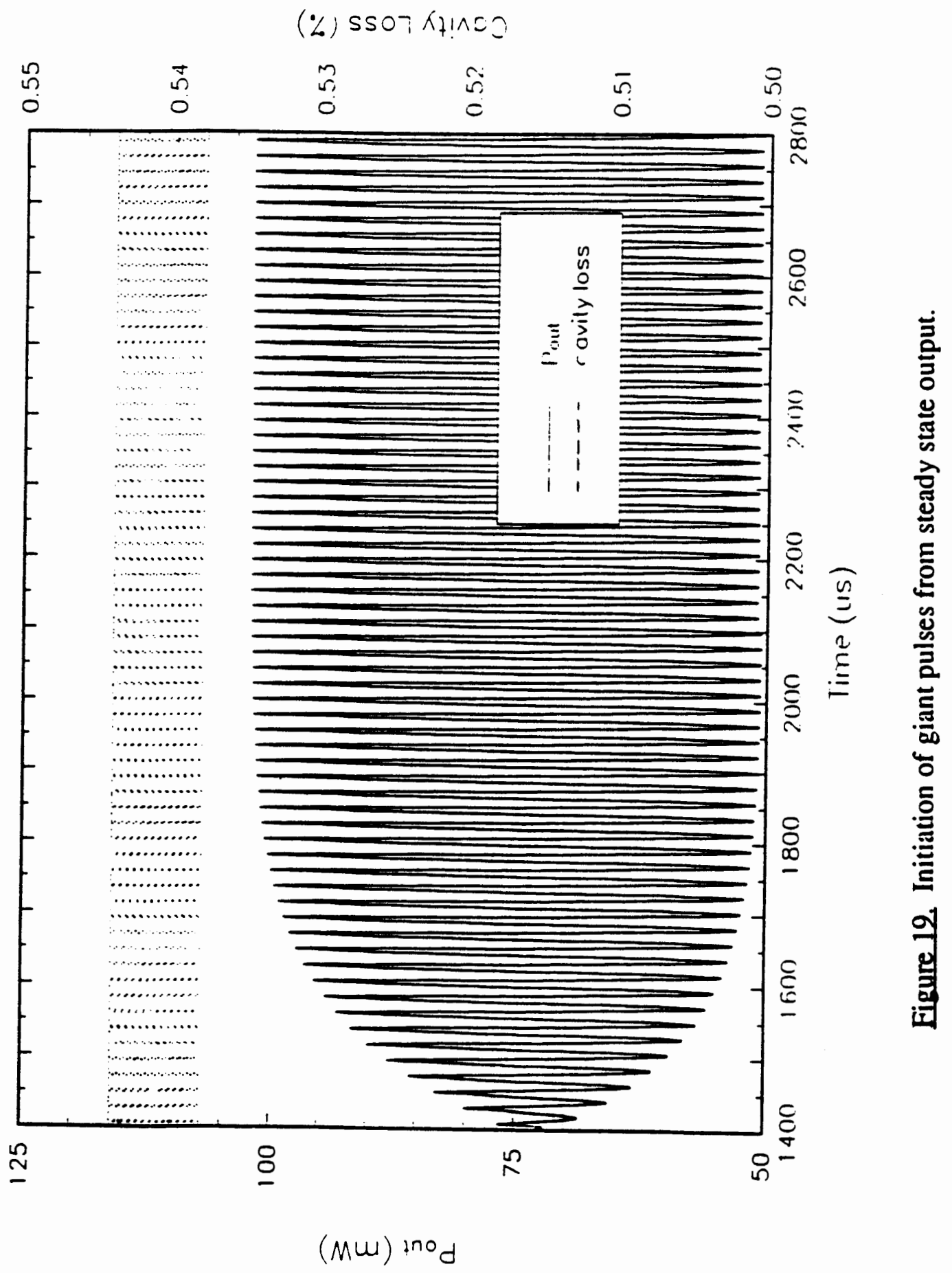




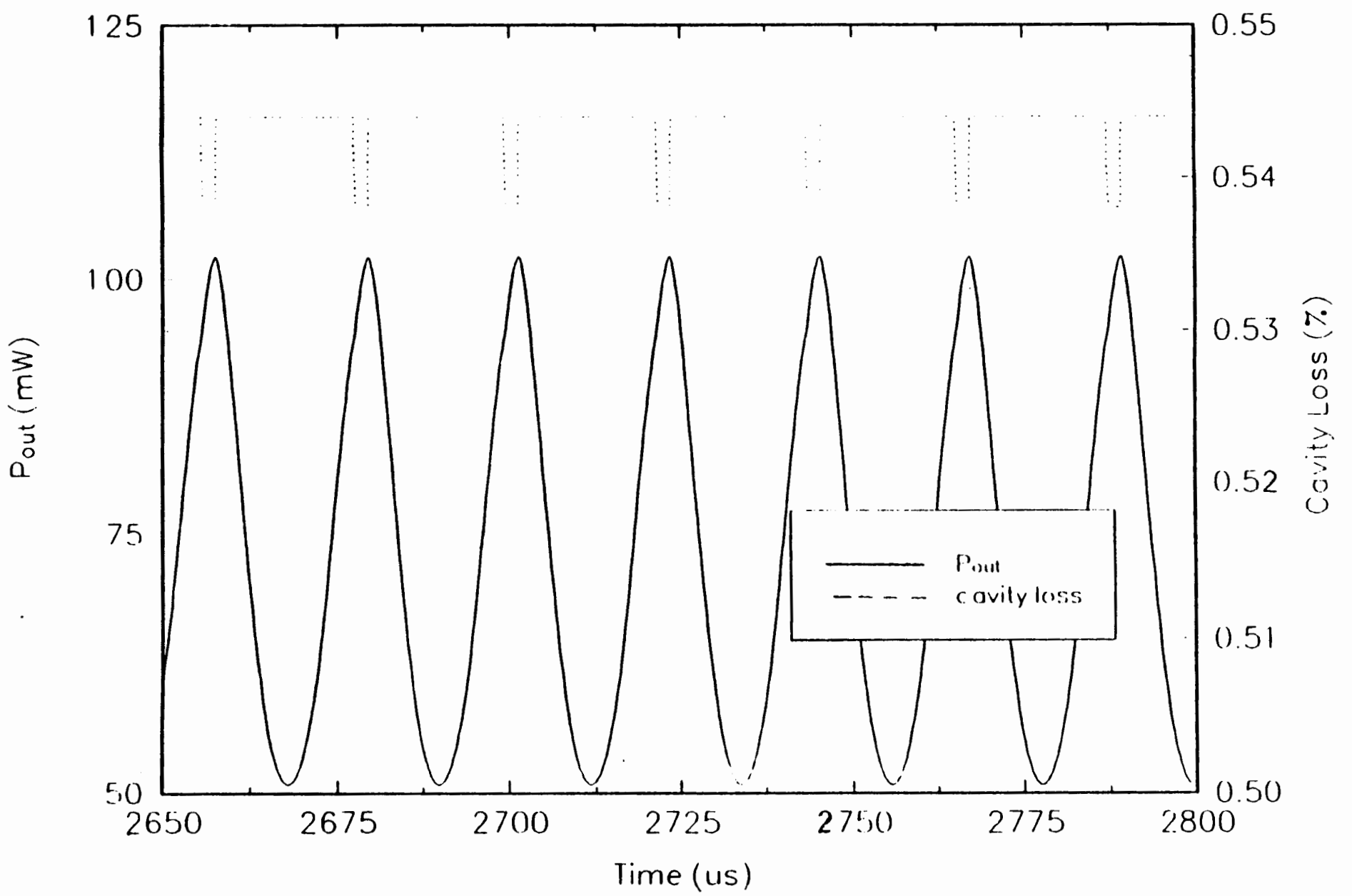

Figure 20. Details of the giant pulses and the cavity loss. 


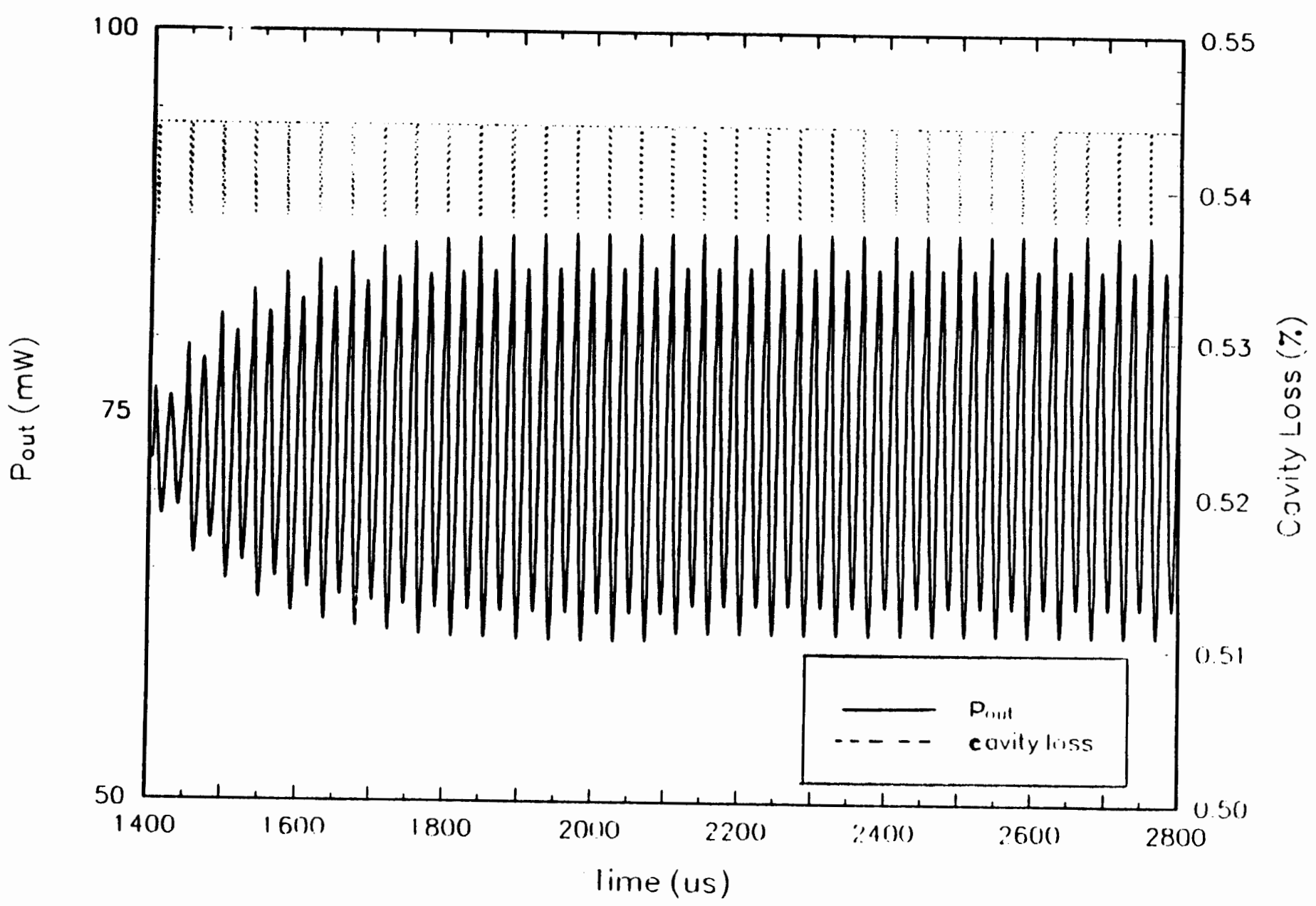

Figure 21A. Laser response to cavity loss modulated at the first subharmonic of the resonance frequency, $\mathrm{f}_{\bmod }=24.009 \mathrm{kHz}$. 


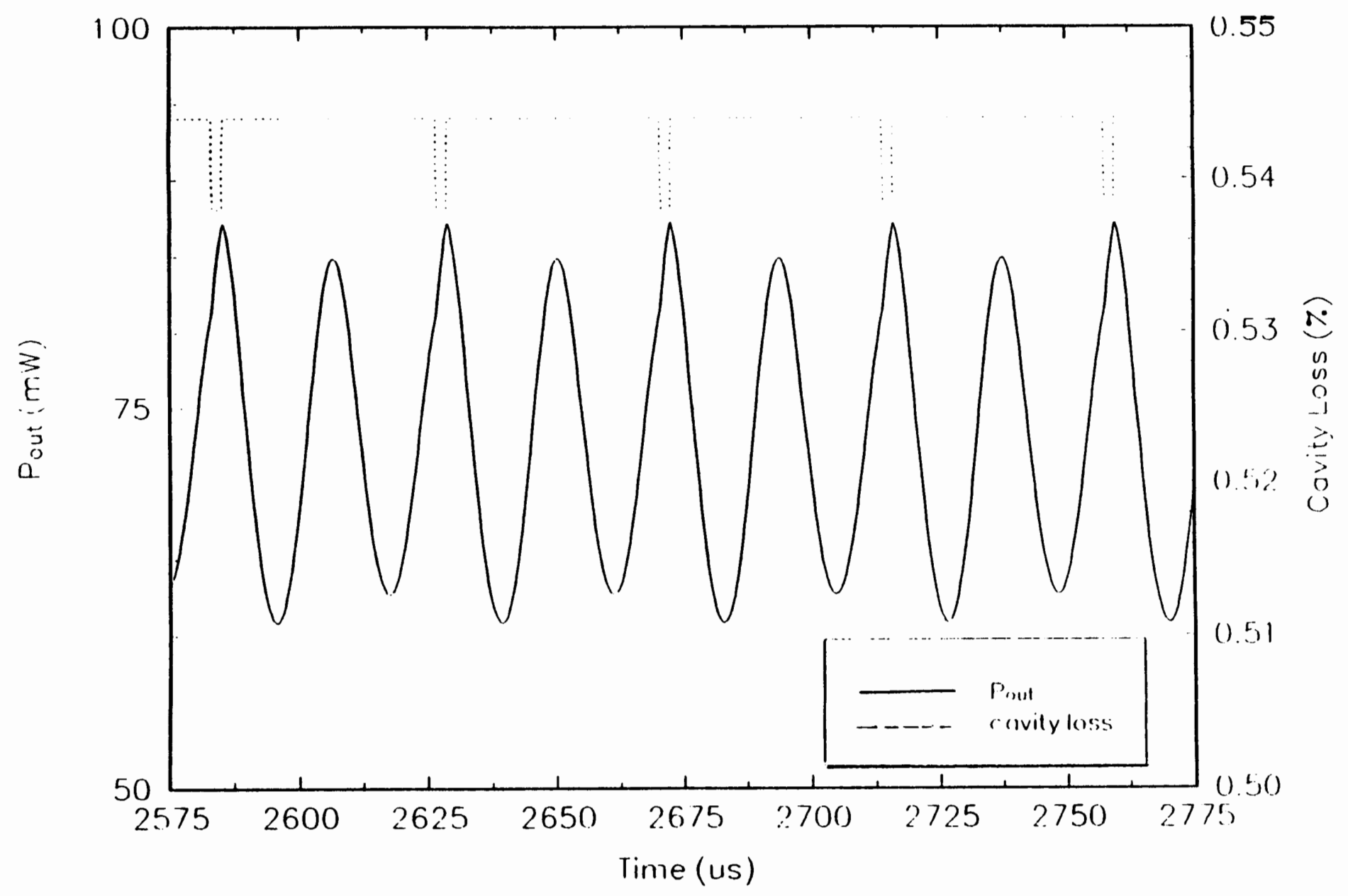

Eigure 21B. Details of the output at $f_{\bmod }=24.009 \mathrm{kHz}$. 


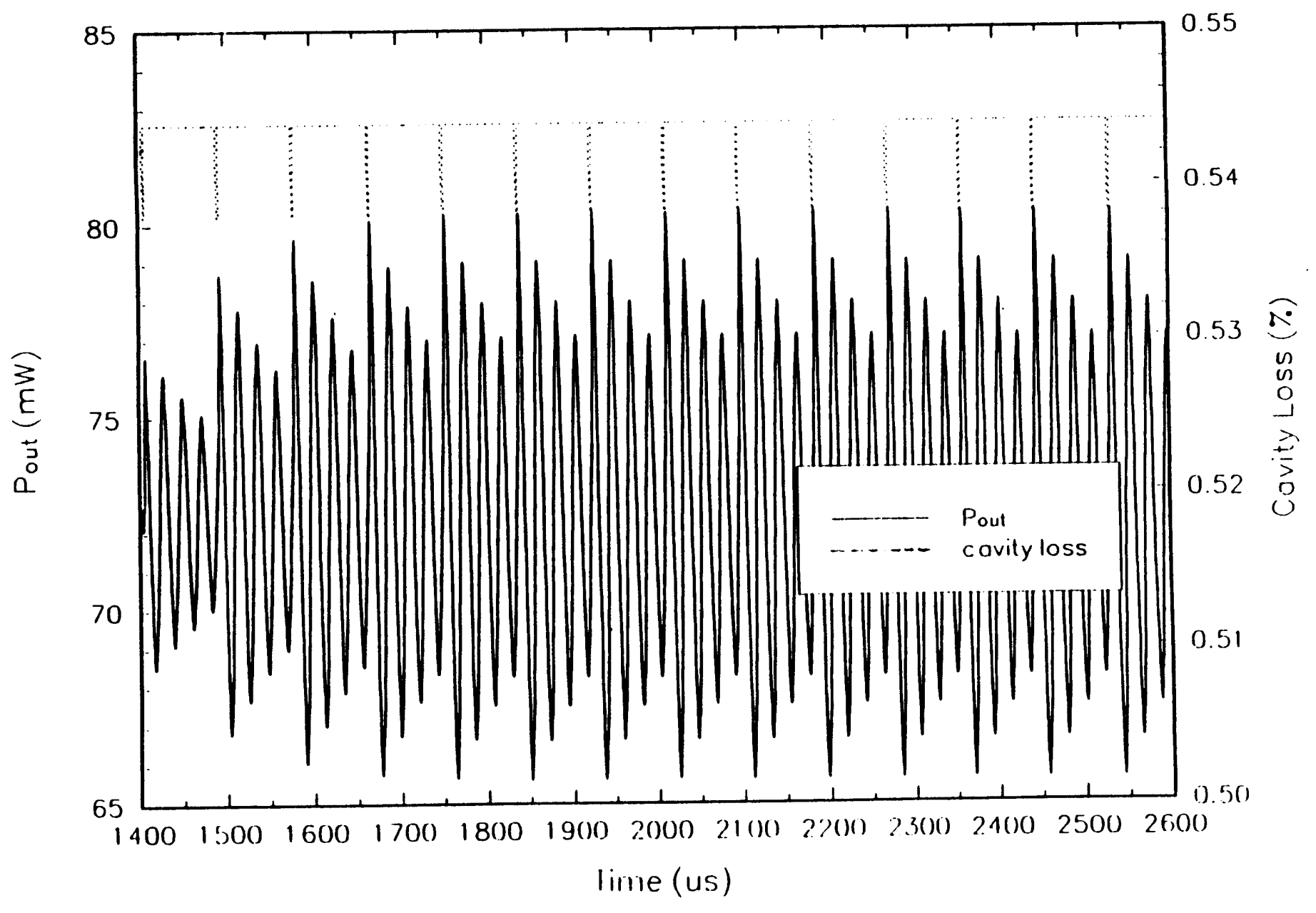

Figure 22A. Laser response to cavity loss modulated at the first subharmonic of the resonance frequency, $\mathrm{f}_{\bmod }=11.818 \mathrm{kHz}$. 


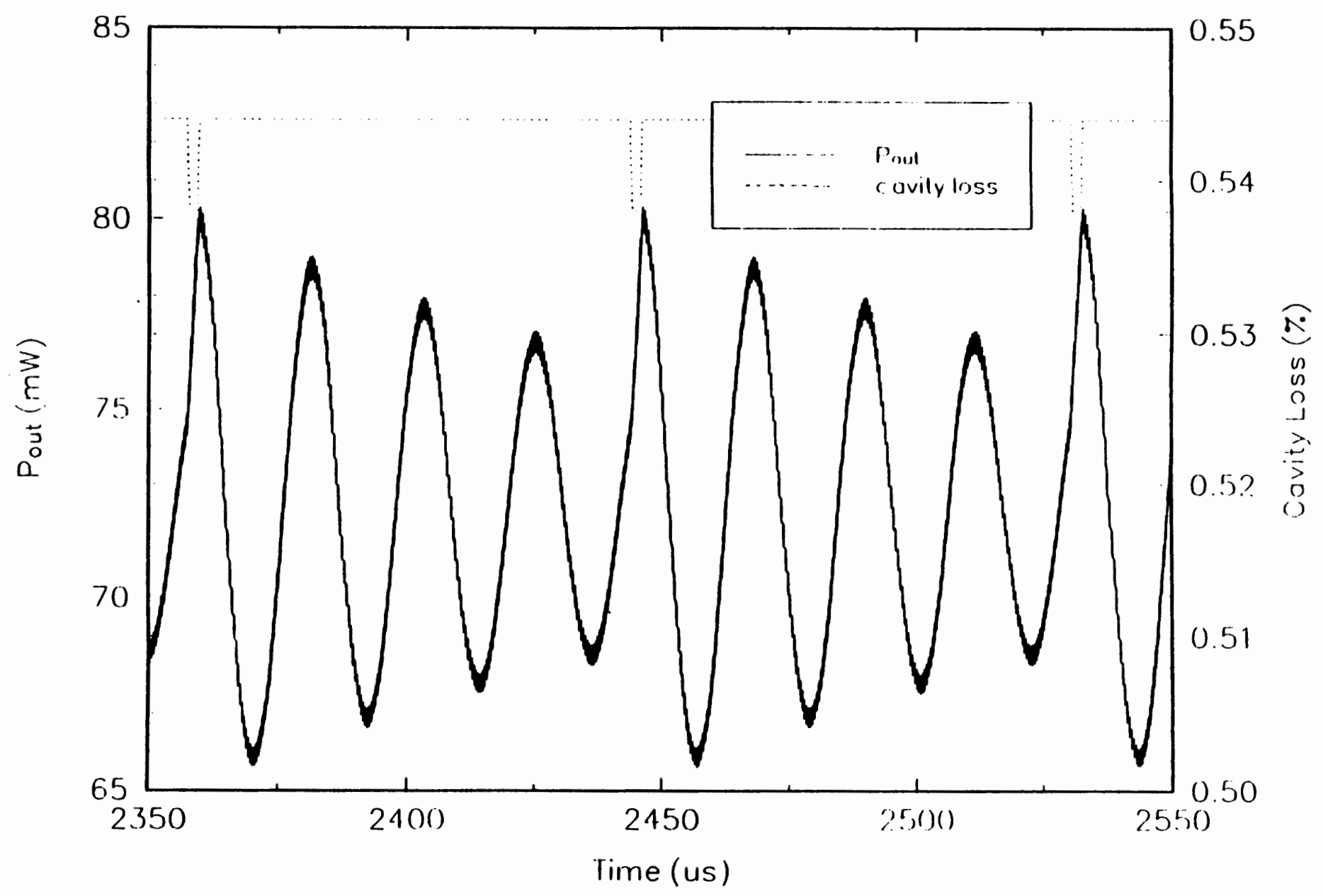

Figure 22B. Details of the output at $f_{\text {mod }}=11.818 \mathrm{kHz}$. 


\section{CHAPTER III}

\section{EXPERIMENTAL RESULTS}

The experimental setup used in the present study is shown in Figure 23A and Figure 23B.

All measurements were taken using a commercially available Q-switched Nd:YAG laser (Model C-95, CVI Corp., Alberqurque, NM). The Nd:YAG rod used was $60 \mathrm{~mm}$ long and $3 \mathrm{~mm}$ in diameter. It contained 1.0 percent neodymium ions by weight. To minimize the reflection losses, both ends of the rods were anti-reflection (AR) coated for operation at $1.064 \mu \mathrm{m}$. The pumping was achieved by focusing the output from two tungsten lamps onto the rod using double-elliptical cylindrical optics. The optical resonator consisted of a concave highly reflective back mirror $(\mathrm{R}>99.98 \%)$ and a partially reflective $(\mathrm{R}=98.93 \%)$ flat output coupler $(\mathrm{OC})$. The reflectivity of the $\mathrm{OC}$ was determined using a Carey 14 spectrophotometer which measures intensity transmission. But because the mirrors used in this laser are dielectric stacked mirrors, and dielectric mirrors can usually be assumed to be lossless, the reflectivity is simply equal to unity minus the transmission. The value of $\operatorname{Roc}=98.93 \%$ was obtained by averaging the results of ten trials. The intracavity loss modulation was achieved by the use of an AR coated acousto-optic modulator (AOM). The AOM was controlled using an external 15 watt Q-switch driver. The controlling signal is an amplitude modulated carrier frequency that operates at 24 MHz. The modulation is a TTL signal which causes the carrier frequency to turn on or off at an adjustable rate between $1 \mathrm{kHz}$ and $30 \mathrm{kHz}$. The duration of the 'off' cycle is fixed at 
$2 \mu \mathrm{s}$. To limit the oscillation to $\mathrm{TEM}_{\infty 0}$ mode, an iris of variable aperture was placed between the AOM and the OC. The cavity gain and losses of the laser were experimentally determined (refer to Appendix B for details).

A number of measures were taken to stabilize the output power of the laser, especially when operating near threshold. The most important of these measures was to operate the two tungsten lamps using a DC power supply (Model DCR150-35A, Sorensen Power Supplies, Norwalk, CN) capable of delivering 200 volts at 45 amps. The output of the Sorensen was monitored using a digital volt meter and a current probe. A second measure was to monitor the temperature of the water used to cool the rod, and operate the laser only when the building water was below $20^{\circ} \mathrm{C}$. Finally, to reduce the intracavity intensity fluctuations arising from air eddy currents, pieces of frosted glass tubing $1 \mathrm{~cm}$ in diameter were placed in regions where the beam was propagating in air.

The transient response of the laser was monitored using a fast silicon detector (Model DET2-Si, Thorlab Inc., Newton, NJ). The detector voltage is proportional to the instantaneous laser output intensity. The output from the detector was monitored using a Tektronix digital oscilloscope (Model 2430A) terminated with a 10K ohm resistor. The average output of the laser was measured using a pyroelectric detector. To keep the detector from giving artificially higher readings due to fluctuations in the room temperature, the detector was enclosed in a cardboard box with a small opening to allow the beam to go through. In addition, the room temperature was kept at $15.5^{\circ} \mathrm{C}$.

\section{Measurements}

Originally, the AOM was designed to be used as a Q-switch. The AOM works by scattering light-waves off an acoustic grating created by electro-acoustic stimulation of a quartz crystal by an RF signal at the crystal resonance frequency. But by inserting a $12 \mathrm{~dB}$ attenuator between the Q-switch driver and the AOM and by minimizing the power deliv- 
ered by the Q-switch driver, the function of the AOM was degraded to a variable loss element rather than a $\mathrm{Q}$-switch. The loss of AOM occurs due to energy loss through scattered beams deflecting off an acoustic grating via Bragg diffraction.

For any pump power, the AOM can be modulated at a frequency such that the output of the laser is a repeated pattern of a series of relaxation oscillations which decay exponentially to some steady state value. Figures $24-37$ show the relaxation oscillations and the subsequent decay to a steady state value for a range of pump powers. The resonant frequency of the relaxation oscillations for each pump power was determined by averaging the frequencies over 10 cycles, just as in Chapter 1 . Measurements show that the frequency of relaxation oscillation increases with pump power as shown in Figure 38. Furthermore, it was shown that if the modulation frequency is set approximately equal to the resonance frequency of relaxation-oscillation, the laser output exhibits a train of spikes at the same frequency as the modulation frequency. If the modulation frequency is approximately $1 / 2$ or $1 / 4$ of the resonance relaxation oscillation frequency, the output is shown to consist of the original spike pattem plus additional components (although smaller in amplitude) located at time intervals equal to the inverse of the driving frequency. If the AOM is driven at twice the resonance frequency, the output is still the same as the spike pattern seen when the AOM is driven at resonance frequency. However, if the drive frequency is anything other than that of sub- or multiple harmonic, the output consist of non repeating pattern of spikes or chaotic output. Figures $39-44$ illustrate the output of a laser where the modulation frequencies are $1 \mathrm{x}, 1 / 2 \mathrm{x}, 1 / 4 \mathrm{x}, 2 \mathrm{x}$, and non-harmonic multiples of the resonant frequency. 


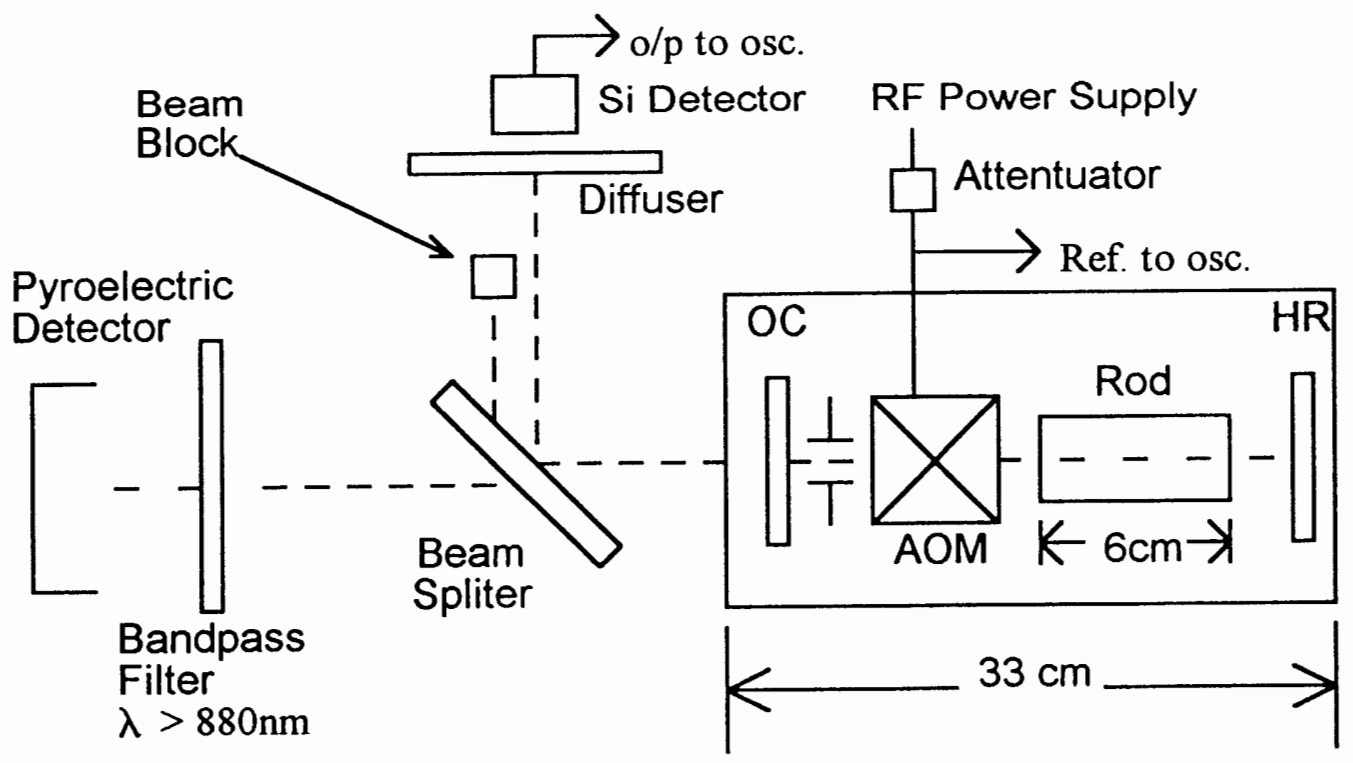

Figure 23A. Schematic diagram of the experimental setup used to study the relaxation oscillations in a $\mathrm{cW} N d$ :YAG laser.

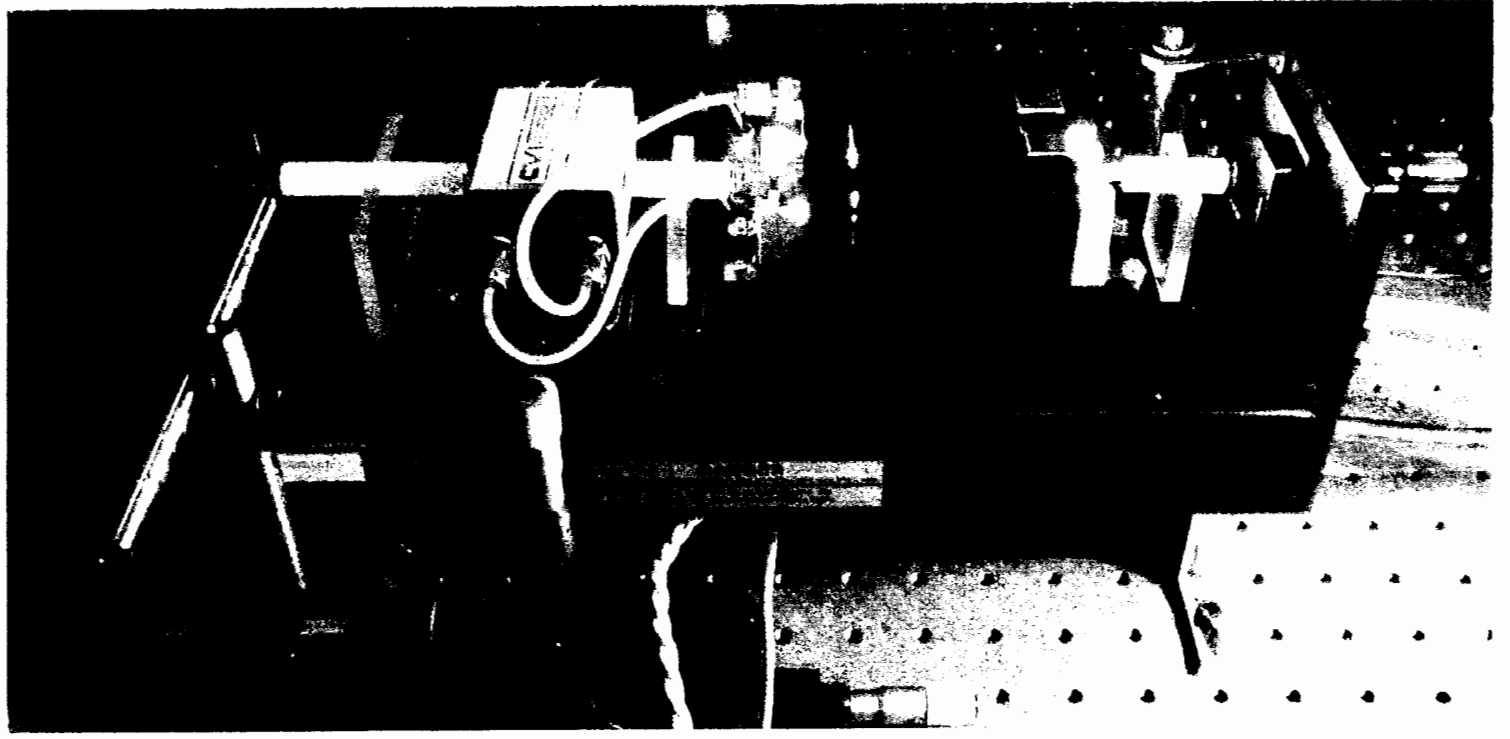

Figure 23B. The CVI laser used for all data acquisition. 

CHI $2 \mathrm{mV}$
A logus $4.34 \mathrm{mV} \quad \mathrm{CH}$

i. $7986 \mathrm{kHz}$

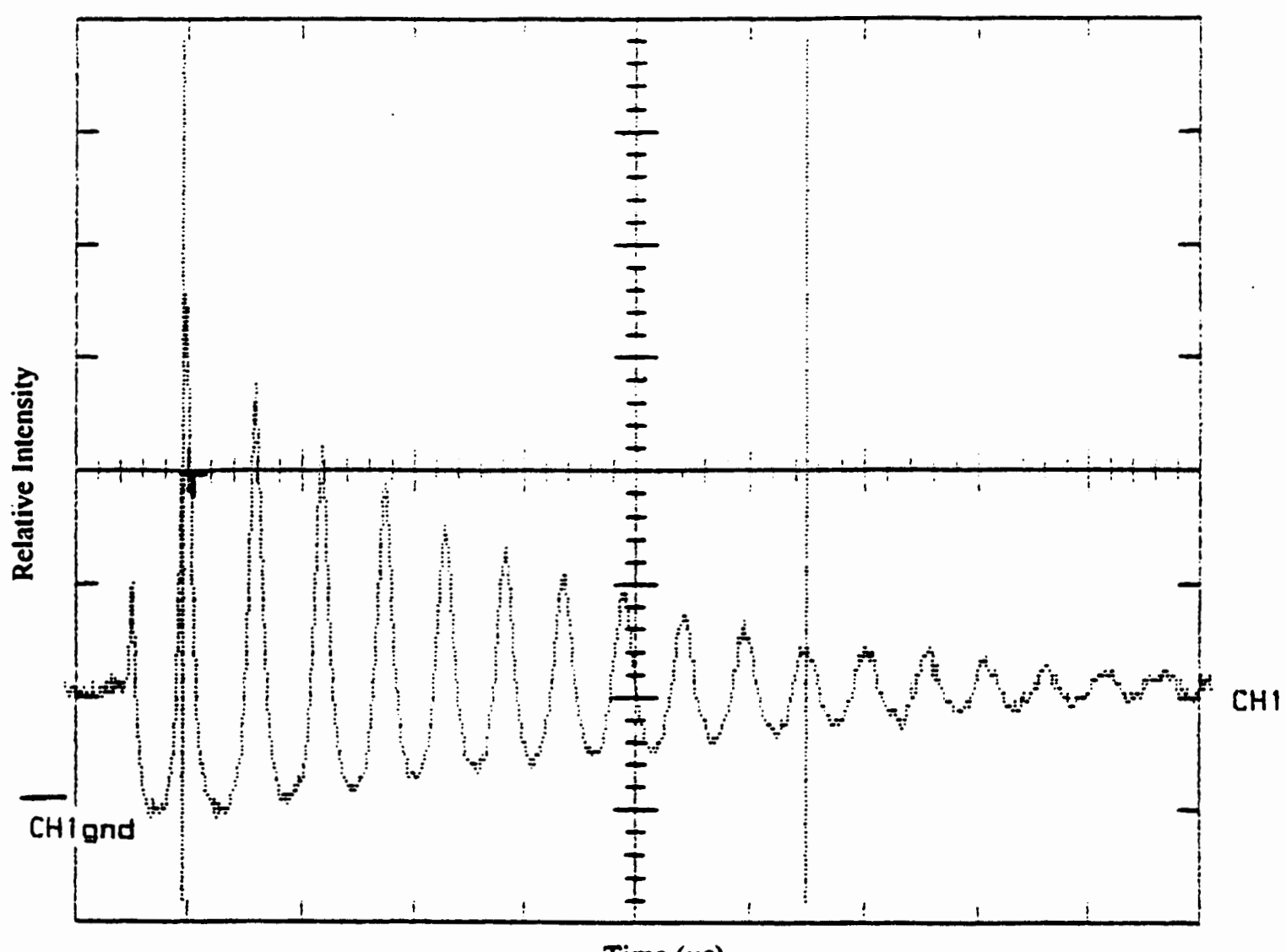

Time $(\mu s)$

Eigure 24. Relaxation oscillation response of Nd:YAG laser pumped at 87.477 $\mathrm{V}_{D C}$ and 11.26 A (flashlamp voltage and current), $P_{n}=I V=985$ $W$. $P_{\text {out }}=7.86 \mathrm{~mW}$, the frequency of relaxation oscillation is $f_{R-0}=17.986$ - $\mathbf{k H z}$ 
$\mathrm{CHI} \quad \mathrm{mV}$

A lobus $3.41 \mathrm{mV} \quad \mathrm{CH}$

$2.0877 \mathrm{kHz}$

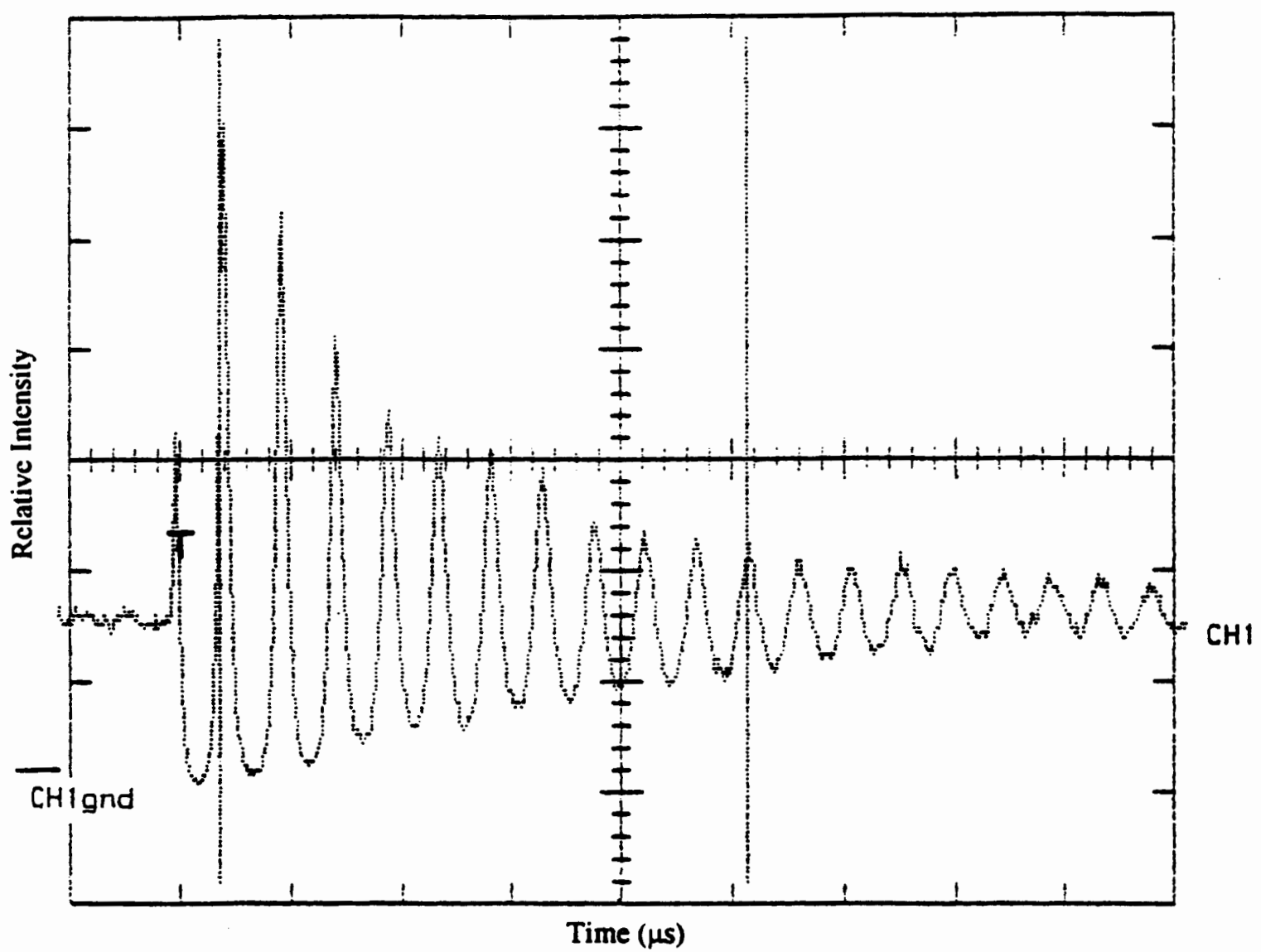

Eigure 25 Laser output when pumped at $\mathrm{V}_{\text {in }}=88.012 \mathrm{~V}, \mathrm{I}_{\text {in }}=11.32 \mathrm{~A}$ which corresponds to $P_{\text {in }}=996.3 \mathrm{~W} . P_{\text {ax }}=11.50 \mathrm{~mW}$, the relaxation oscillation frequency is $\mathrm{f}_{\mathrm{R}-\mathrm{O}}=20.877 \mathrm{kHz}$. 

$\mathrm{CHI} \quad 5 \mathrm{mV}$
A robus
$6.21 \mathrm{mV}$
$\mathrm{CHI}$
$2.5600 \mathrm{kHz}$

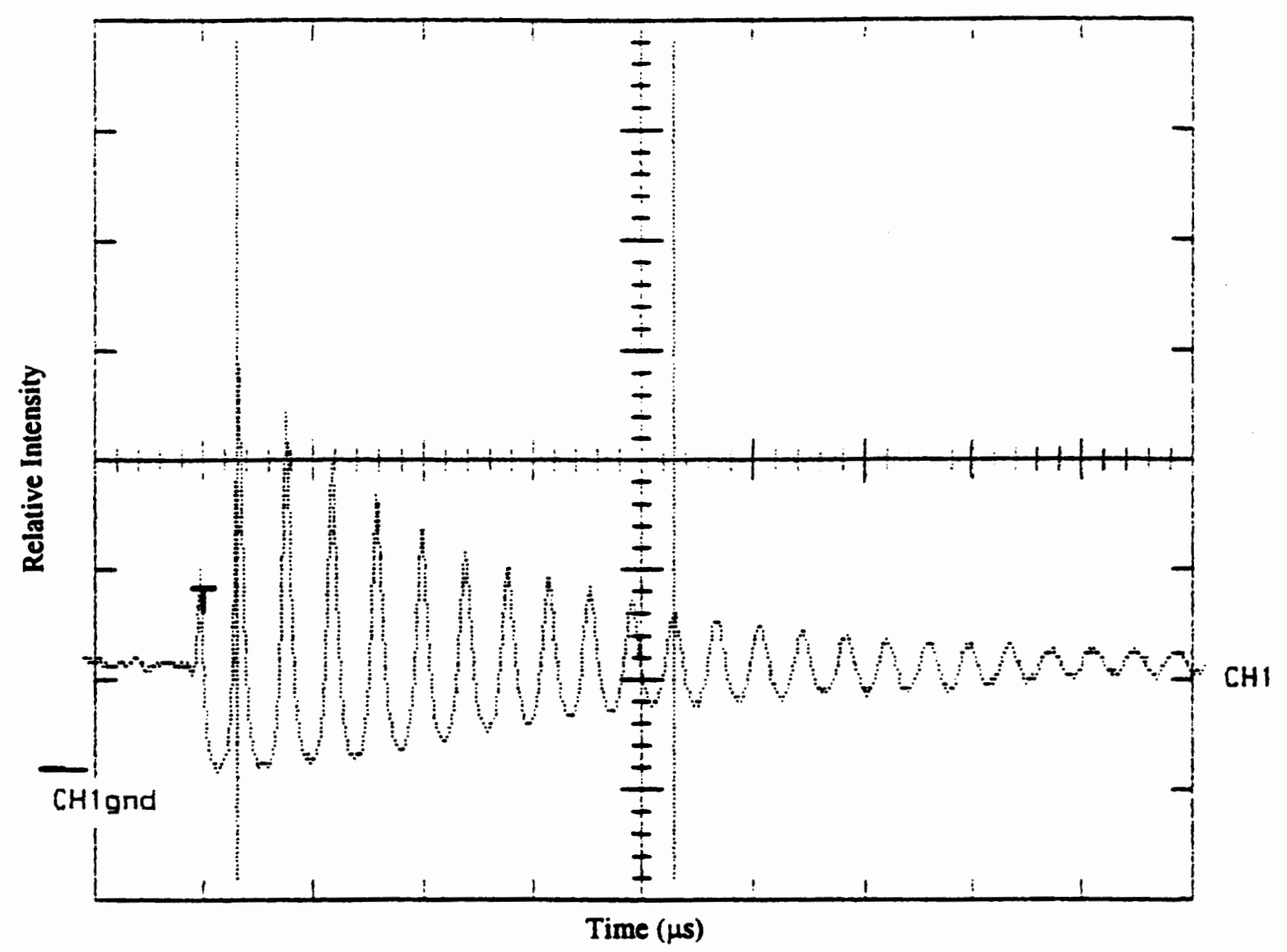

Eigure 26. Laser output when pumped at $\mathrm{V}_{\text {in }}=89.007 \mathrm{~V}, \mathrm{I}_{\text {in }}=11.38 \mathrm{~A}$ which corresponds to $P_{\text {in }}=1012.9 \mathrm{~W}$. $P_{\text {out }}=16.83 \mathrm{~mW}$, the relaxation oscillation frequency is $\mathfrak{R}_{\mathrm{R}-\mathrm{O}}=25.000 \mathrm{kHz}$. 
$\mathrm{CHI} 5 \mathrm{mV}$ A loous $6.21 \mathrm{mV} \quad \mathrm{CH}$

$2.6667 \mathrm{kHz}$

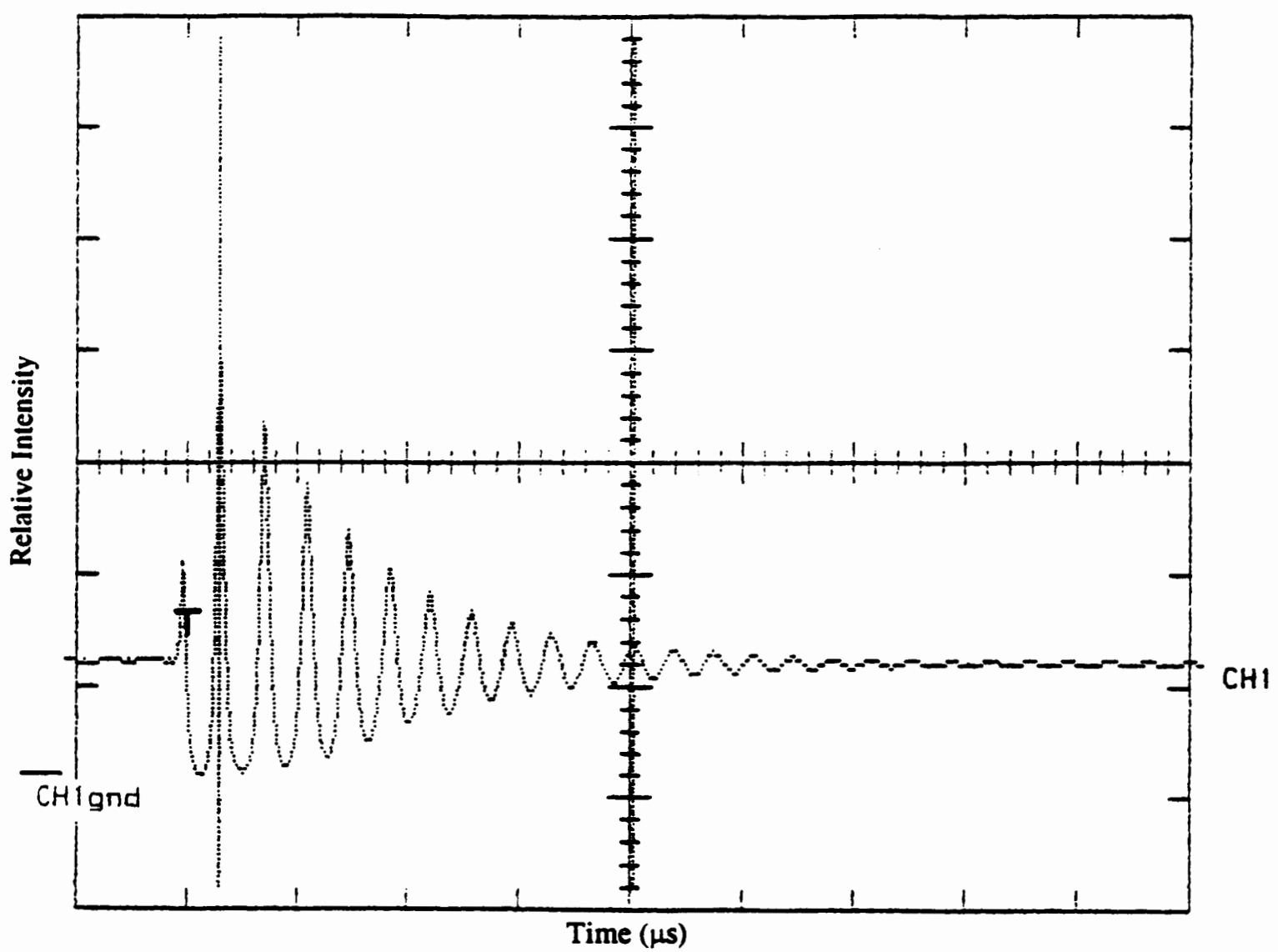

Eigure 27. Laser output when pumped at $\mathrm{V}_{\text {in }}=89.996 \mathrm{~V}, \mathrm{I}_{\text {in }}=11.44 \mathrm{~A}$ which corresponds to $P_{\text {in }}=1029.55 \mathrm{~W} . \mathrm{P}_{\text {oux }}=21.32 \mathrm{~mW}$, the relaxation oscillation frequency is $\mathrm{f}_{\mathrm{R}-\mathrm{O}}=26.667 \mathrm{kHz}$. 
$\mathrm{CHI} 5 \mathrm{mV} \quad \mathrm{A}$ 50us $7.03 \mathrm{mV} \quad \mathrm{CH}$

$2.8653 \mathrm{k} \mathrm{Hz}$

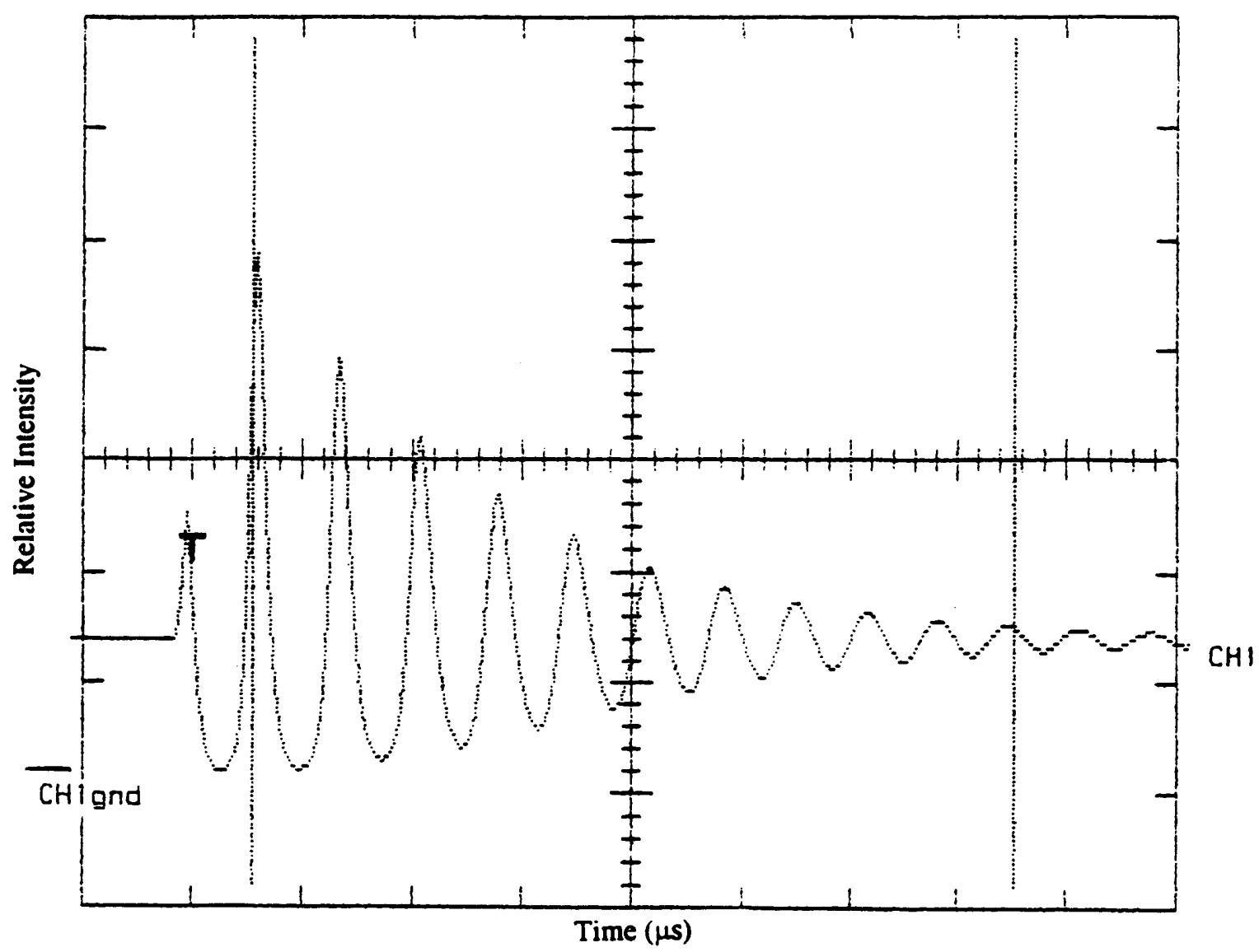

Eigure 28. Laser output when pumped at $V_{\text {in }}=91.006 \mathrm{~V}, I_{\text {in }}=11.52 \mathrm{~A}$ which corresponds to $P_{\text {in }}=1048.39 \mathrm{~W} . P_{\text {our }}=27.49 \mathrm{~mW}$, the relaxation oscillation frequency is $\mathrm{f}_{\mathrm{R}-\mathrm{O}}=28.653 \mathrm{kHz}$. 


$\mathrm{CHI} 5 \mathrm{mV}$ A $5015 \mathrm{i} \quad 0.0 \mathrm{mV} \mathrm{CH}$
$3.0769 \mathrm{kHz}$

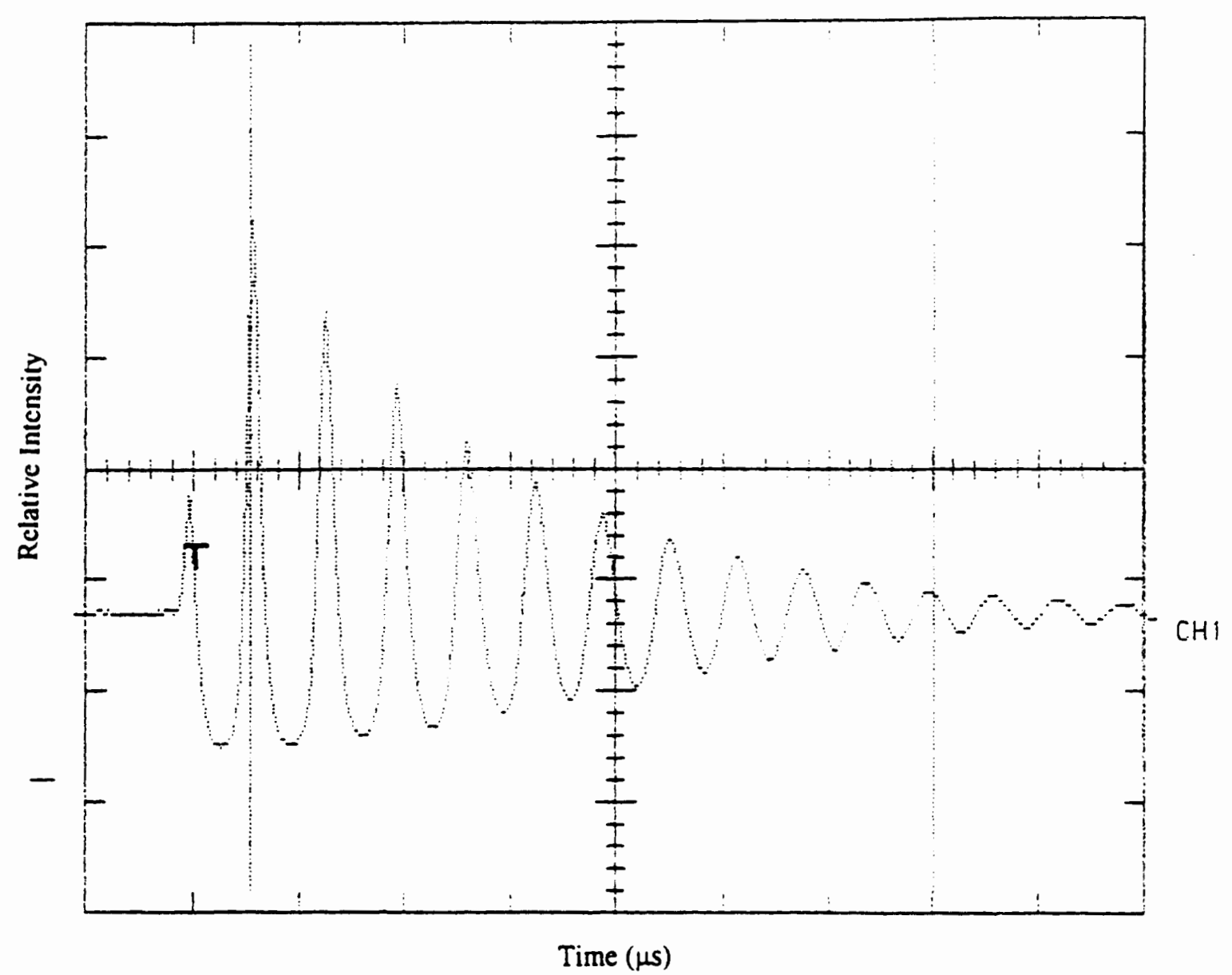

Eigure 29. Laser output when pumped at $\mathrm{V}_{\text {in }}=92.003 \mathrm{~V}, \mathrm{I}_{\mathrm{in}}=11.57 \mathrm{~A}$

- which corresponds to $\mathrm{P}_{\text {in }}=1058.22 \mathrm{~W}$. $\mathrm{P}_{\text {our }}=33.10 \mathrm{~mW}$, the relaxation oscillation frequency is $f_{R-0}=30.769 \mathrm{kHz}$. 
$\mathrm{CH}$ SmV A loDus $7.03 \mathrm{mV}$ CHI

3. $3223 k+12$

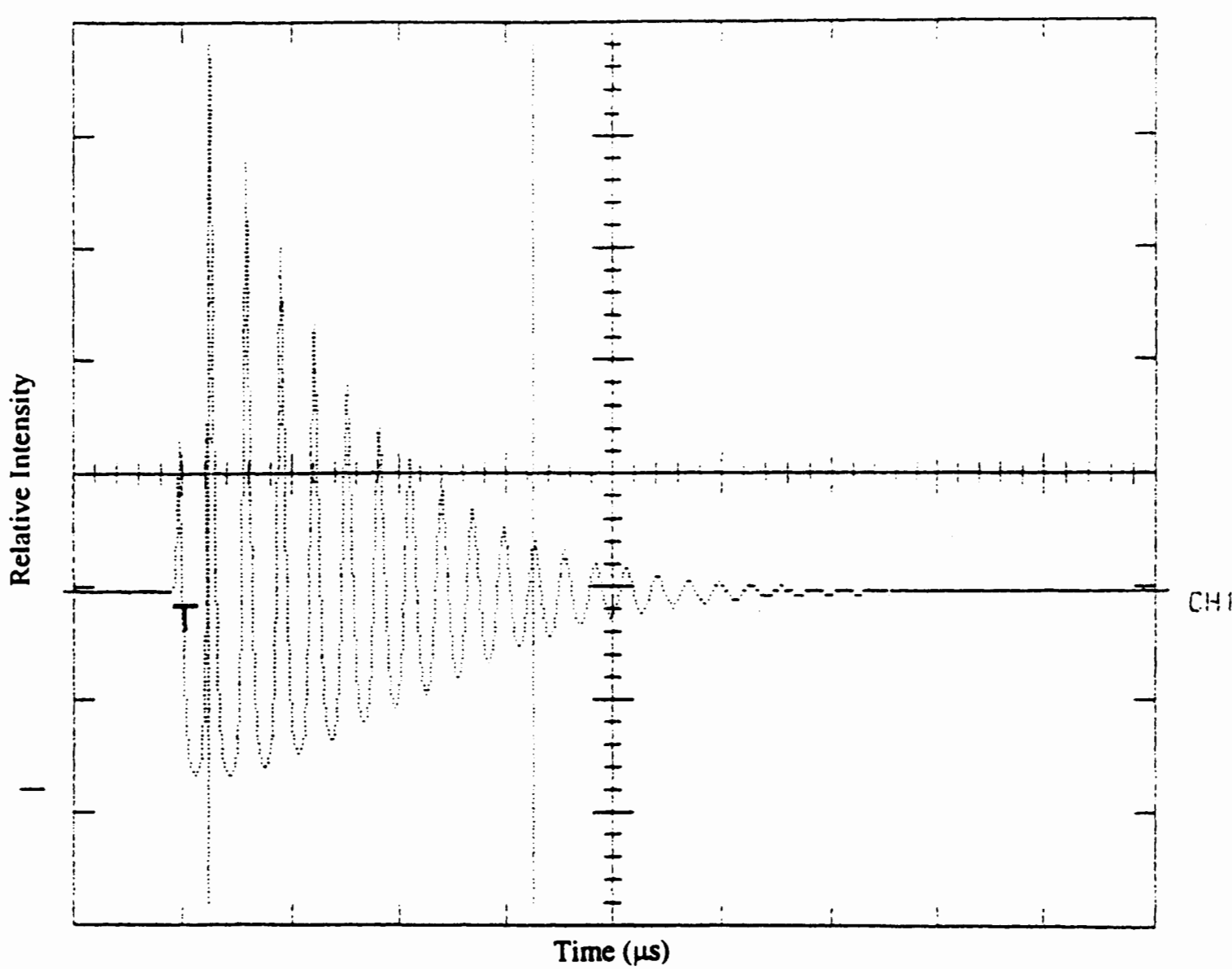

Eiqure 30. Laser output when pumped at $\mathrm{V}_{\mathrm{in}}=93.008 \mathrm{~V}, \mathrm{I}_{\mathrm{in}}=11.65 \mathrm{~A}$

- which corresponds to $P_{\text {in }}=1083.54 \mathrm{~W} . \mathrm{P}_{\text {oux }}=37.59 \mathrm{~mW}$, the relaxation oscillation frequency is $f_{R-0}=33.223 \mathrm{kHz}$. 
$\mathrm{CHI} 5 \mathrm{mV}$ A leOUs $7.03 \mathrm{mV} \quad \mathrm{CH}$

$3.4965 \mathrm{kHz}$

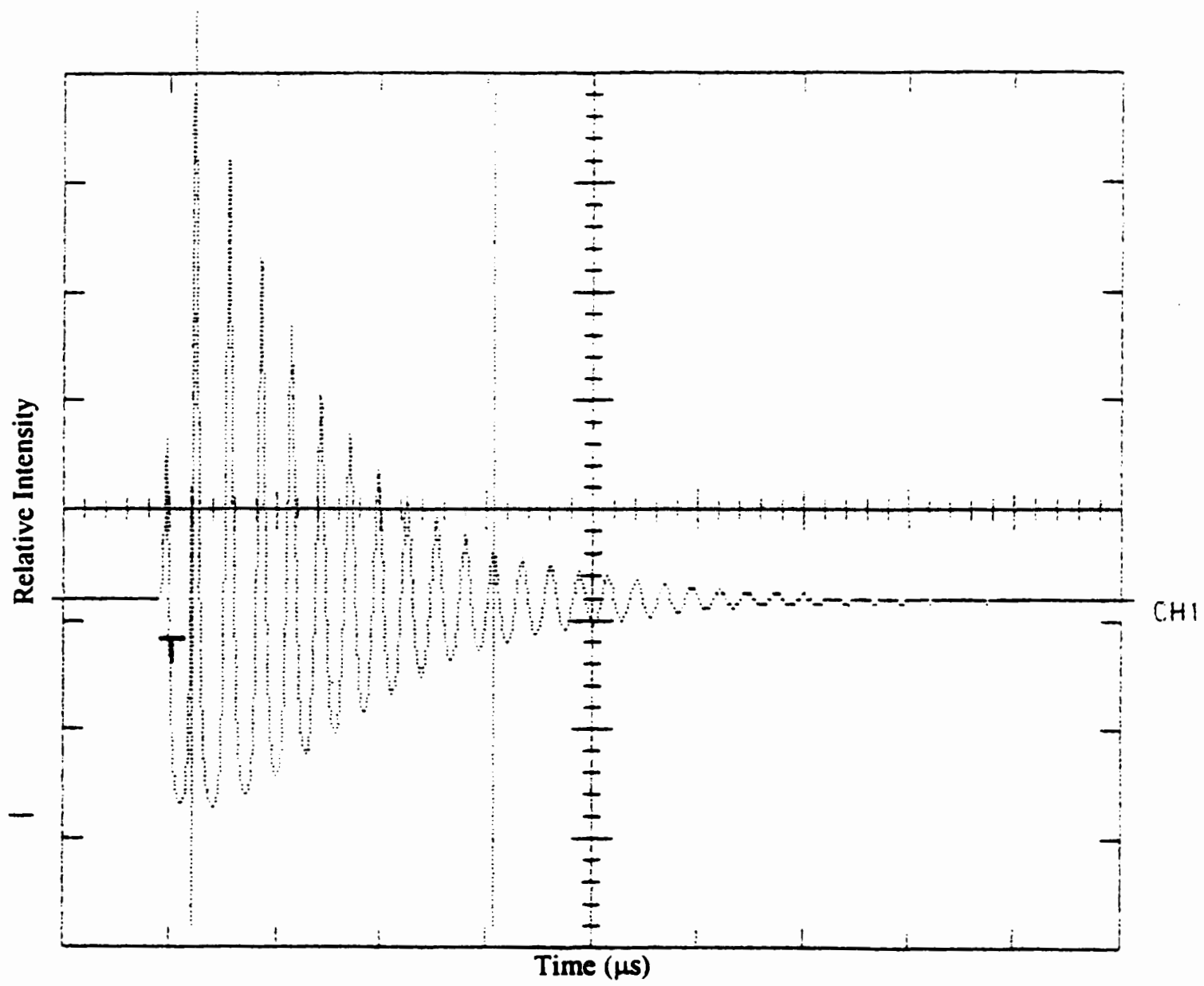

Eiqure 31. Laser output when pumped at $V_{\text {in }}=94.013 \mathrm{~V}, I_{\text {in }}=11.72 \mathrm{~A}$ which corresponds to $P_{\text {in }}=1101.83 \mathrm{~W} . P_{\text {out }}=43.76 \mathrm{~mW}$, the relaxation

- oscillation frequency is $f_{R-0}=34.965 \mathrm{kHz}$. 
$\mathrm{CHH} \quad 10 \mathrm{mV}$

A 100us $7.03 \mathrm{mV} \quad \mathrm{CH}$

$3.7453 \mathrm{kHz}$

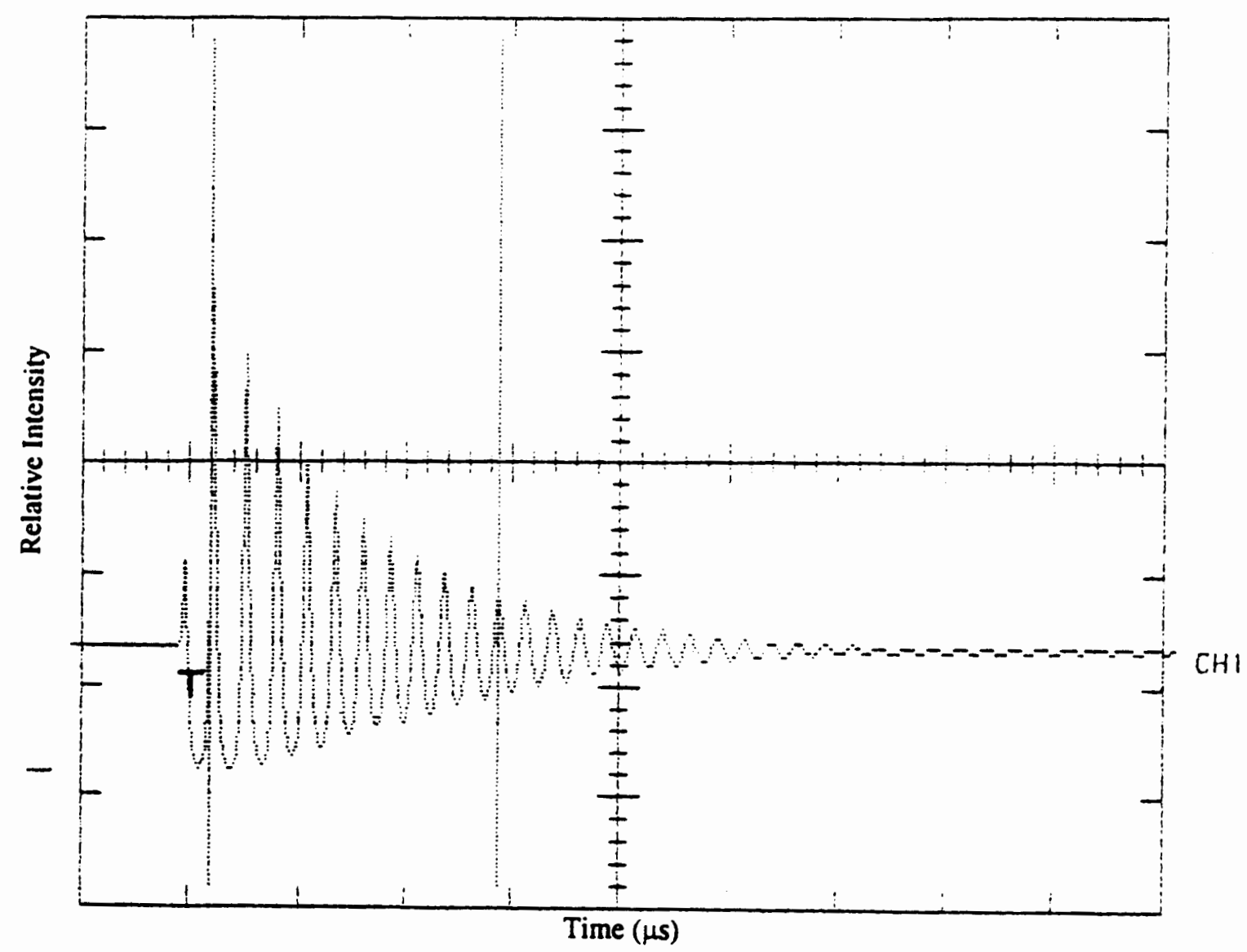

Eiqure 32. Laser output when pumped at $V_{\text {in }}=95.008 \mathrm{~V}, I_{\text {in }}=11.79 \mathrm{~A}$ which corresponds to $P_{i n}=1120.14 \mathrm{~W}$. $P_{\text {out }}=47.69 \mathrm{~mW}$, the relaxation

- oscillation frequency is $\mathrm{f}_{\mathrm{R} \cdot \mathrm{O}}=37.453 \mathrm{kHz}$. 
$\mathrm{CHI} 10 \mathrm{mV}$ fi loGus $7.03 \mathrm{mV} \quad \mathrm{CHI}$

$3.8023 \mathrm{kHz}$

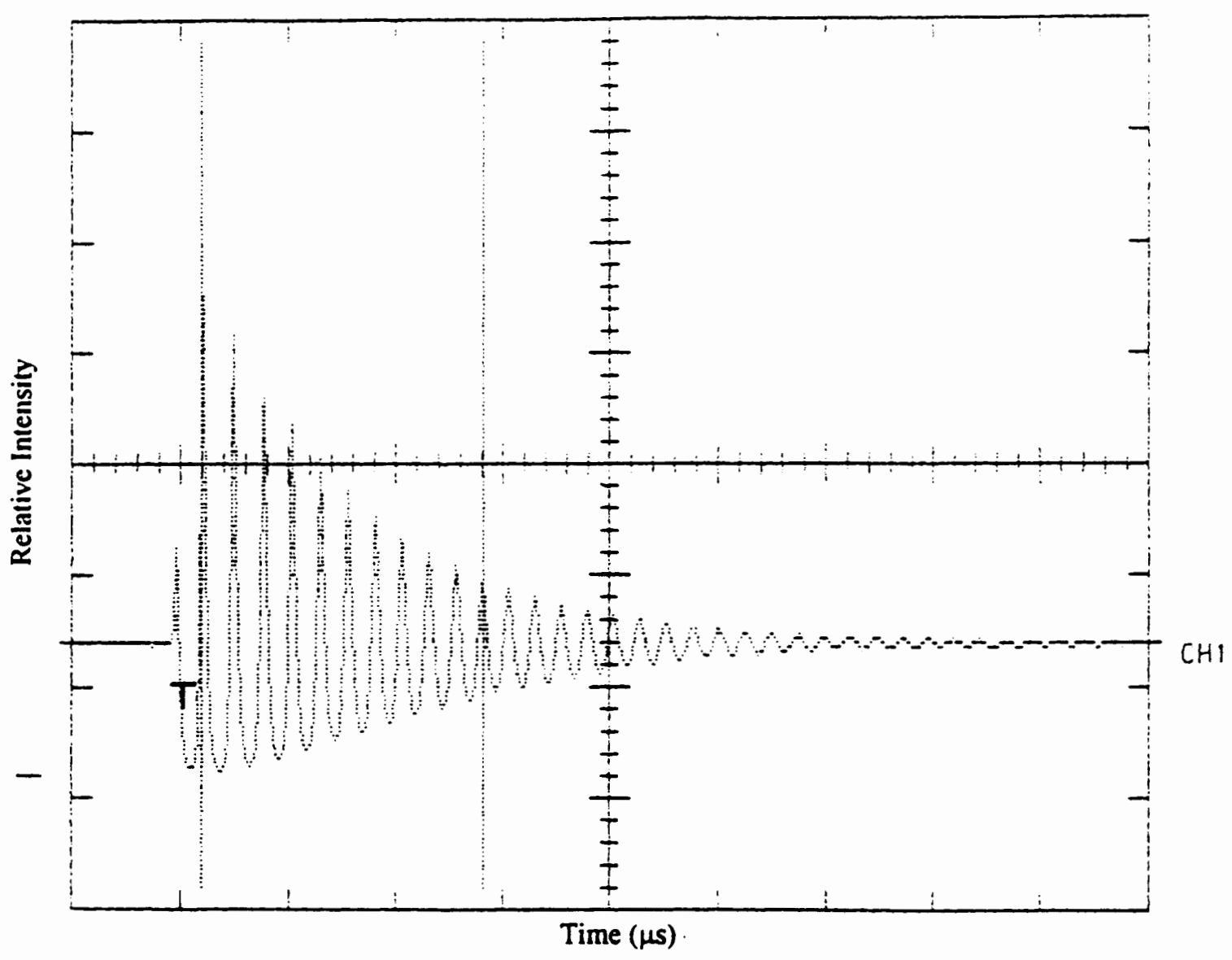

Eigure 33. Laser output when pumped at $\mathrm{V}_{\text {in }}=96.002 \mathrm{~V}, \mathrm{I}_{\text {in }}=11.86 \mathrm{~A}$ which corresponds to $P_{i n}=1138.58 \mathrm{~W} . P_{\text {our }}=52.74 \mathrm{~mW}$, the relaxation - oscillation frequency is $\mathrm{f}_{\mathrm{R} \cdot \mathrm{O}}=38.023 \mathrm{kHz}$. 
CHI $10 \mathrm{mV}$

G $100 \mathrm{us} 7.03 \mathrm{mV} \mathrm{CH}$

$4.0486 \mathrm{kHz}$

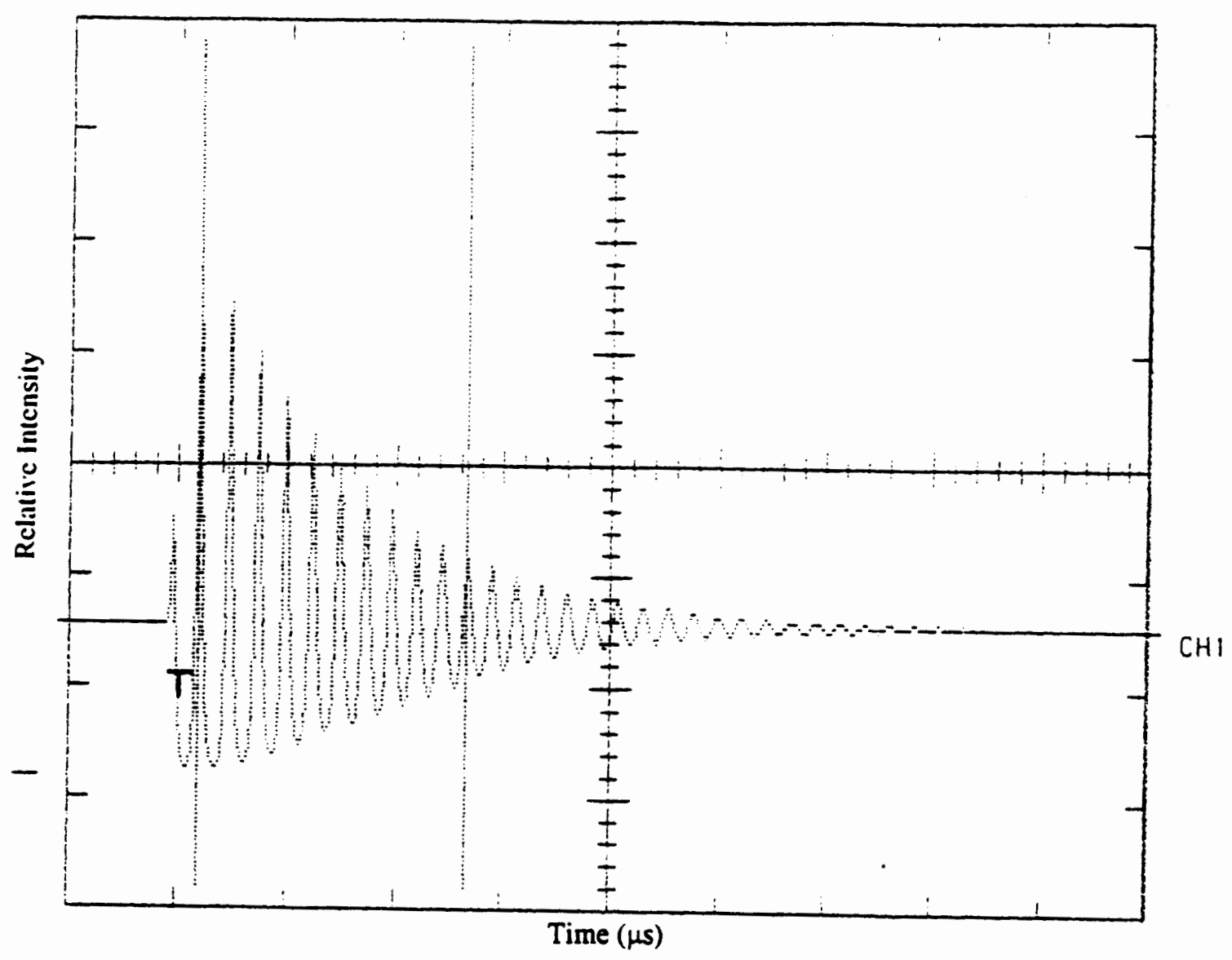

Eigure 34. Laser output when pumped at $\mathrm{V}_{\text {in }}=97.006 \mathrm{~V}, \mathrm{I}_{\text {in }}=11.93 \mathrm{~A}$ which corresponds to $P_{\text {in }}=1157.28 \mathrm{~W} . \mathrm{P}_{\text {out }}=59.47 \mathrm{~mW}$, the relaxation - oscillation frequency is $\mathrm{f}_{\mathrm{R}-\mathrm{O}}=40.486 \mathrm{kHz}$. 

CHI 10mV
A 100us $7.03 \mathrm{mV} \quad \mathrm{CH} 1$
$4.2194 \mathrm{kHz}$

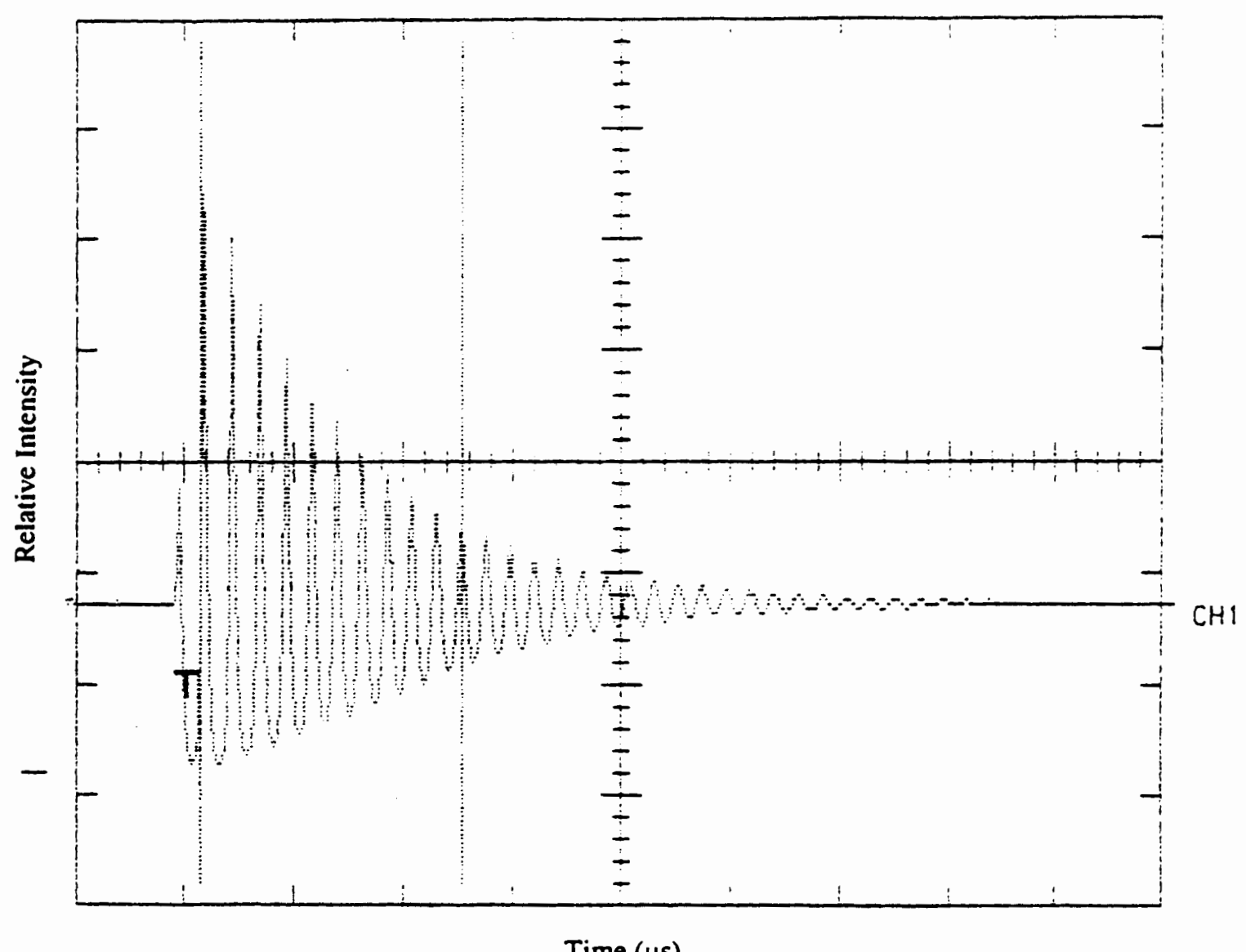

Eigure 35. Laser output when pumped at $\mathrm{V}_{\mathrm{in}}=98.025 \mathrm{~V}, \mathrm{I}_{\mathrm{nn}}=11.99 \mathrm{~A}$ which corresponds to $P_{\text {in }}=1175.32 \mathrm{~W}$. $P_{\text {out }}=66.21 \mathrm{~mW}$, the relaxation

- oscillation frequency is $f_{R-O}=42.194 \mathrm{kHz}$. 
$\mathrm{CH} 10 \mathrm{mV}$

A $100 \mathrm{us} 7.03 \mathrm{mV} \quad \mathrm{CHI}$

$4.4053 \mathrm{kHz}$

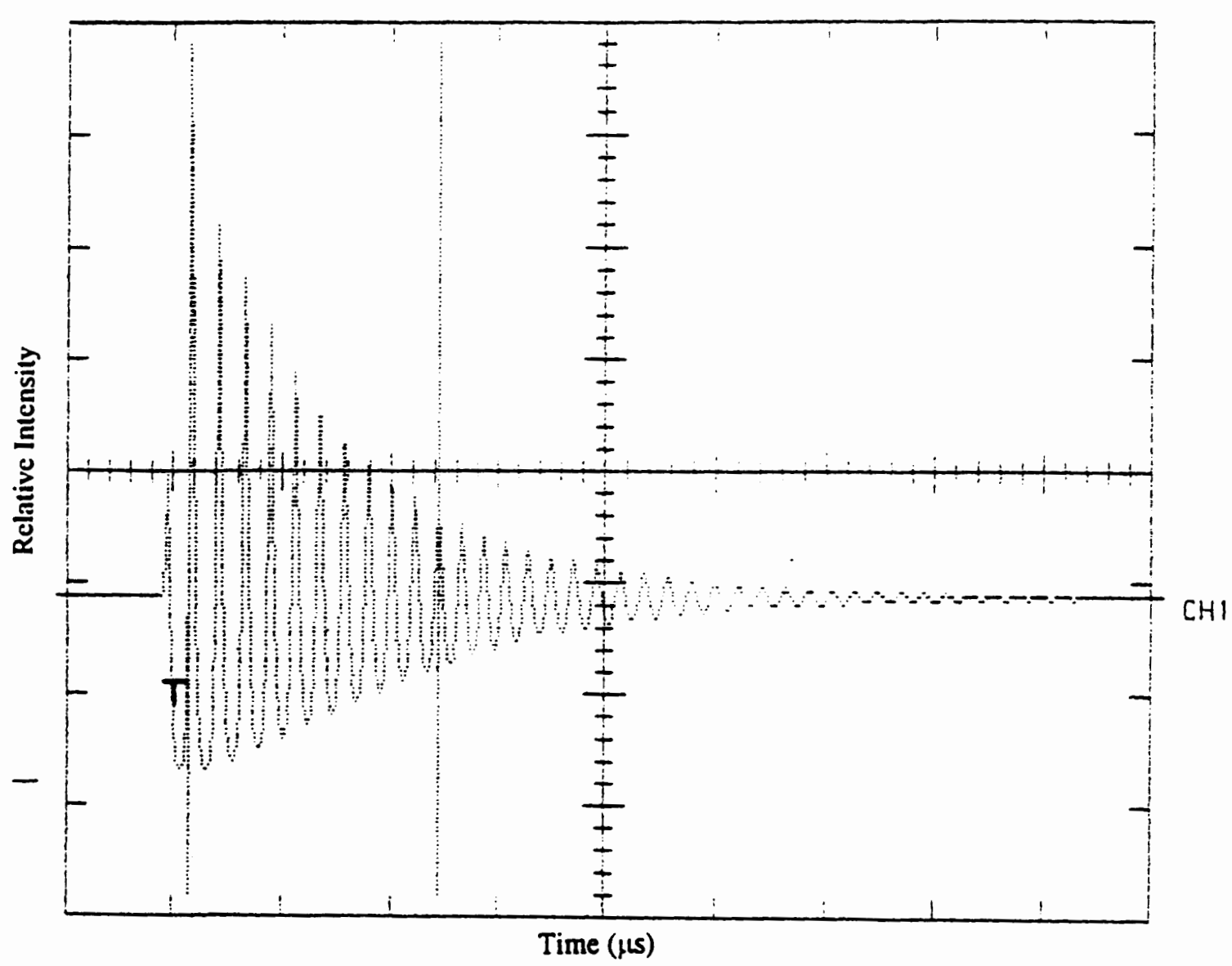

Eigure 36 Laser output when pumped at $\mathrm{V}_{\text {in }}=99.018 \mathrm{~V}, \mathrm{I}_{\text {in }}=12.05 \mathrm{~A}$ which corresponds to $P_{\text {in }}=1193.17 \mathrm{~W} . P_{\text {out }}=71.82 \mathrm{~mW}$, the relaxation

- oscillation frequency is $f_{R-0}=44.053 \mathrm{kHz}$. 
CHI lomV A logus $7.03 \mathrm{mV}$ CHI

4. $5872 k+1 z$

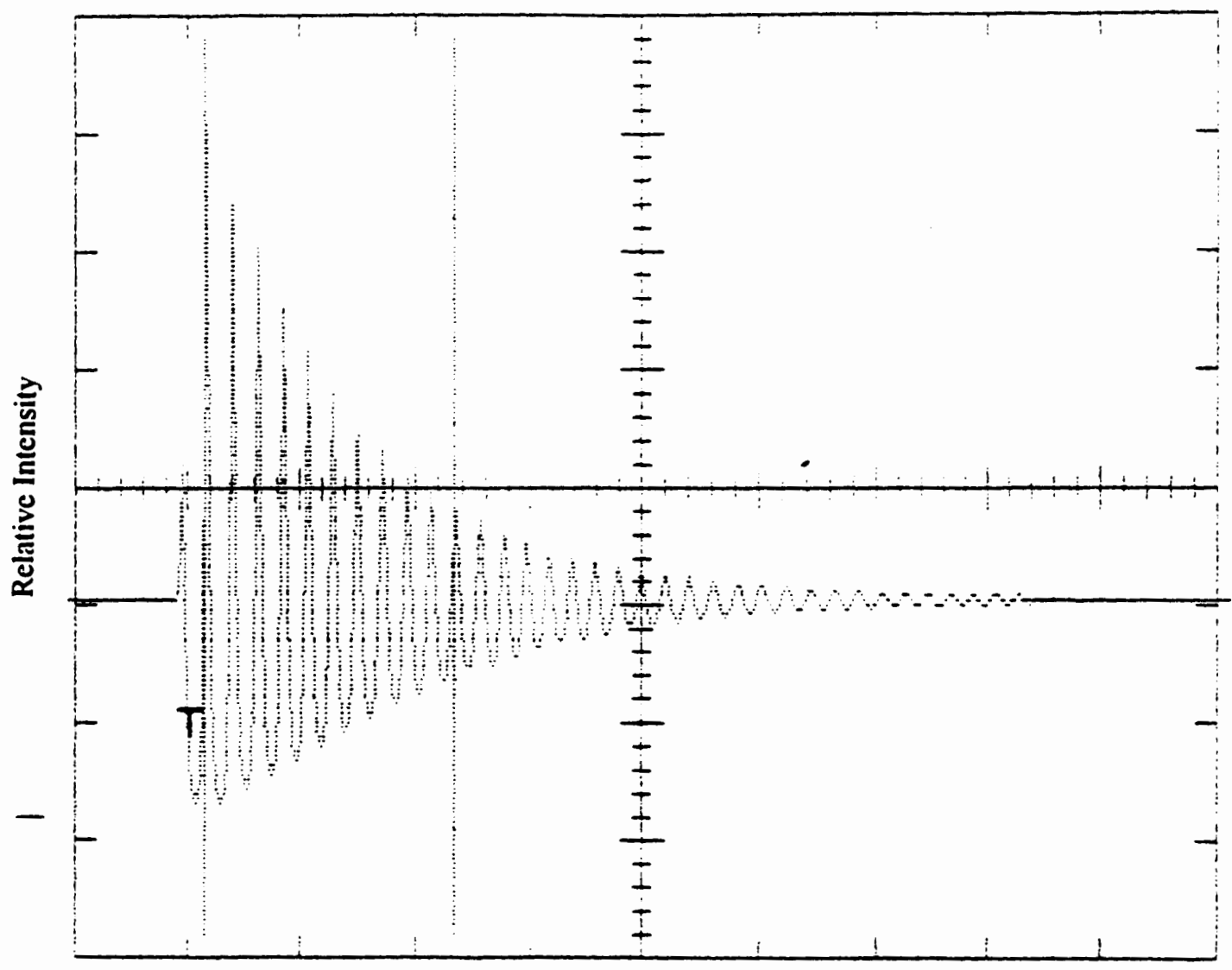

Time $(\mu s)$

Eigure 37 Laser output when pumped at $V_{\text {in }}=100.008 \mathrm{~V}, I_{\text {in }}=12.12 \mathrm{~A}$ which corresponds to $P_{\text {in }}=1212.10 \mathrm{~W} . \mathrm{P}_{\text {out }}=77.43 \mathrm{~mW}$, the relaxation oscillation frequency is $\mathrm{f}_{\mathrm{R}-\mathrm{O}}=45.872 \mathrm{kHz}$. 


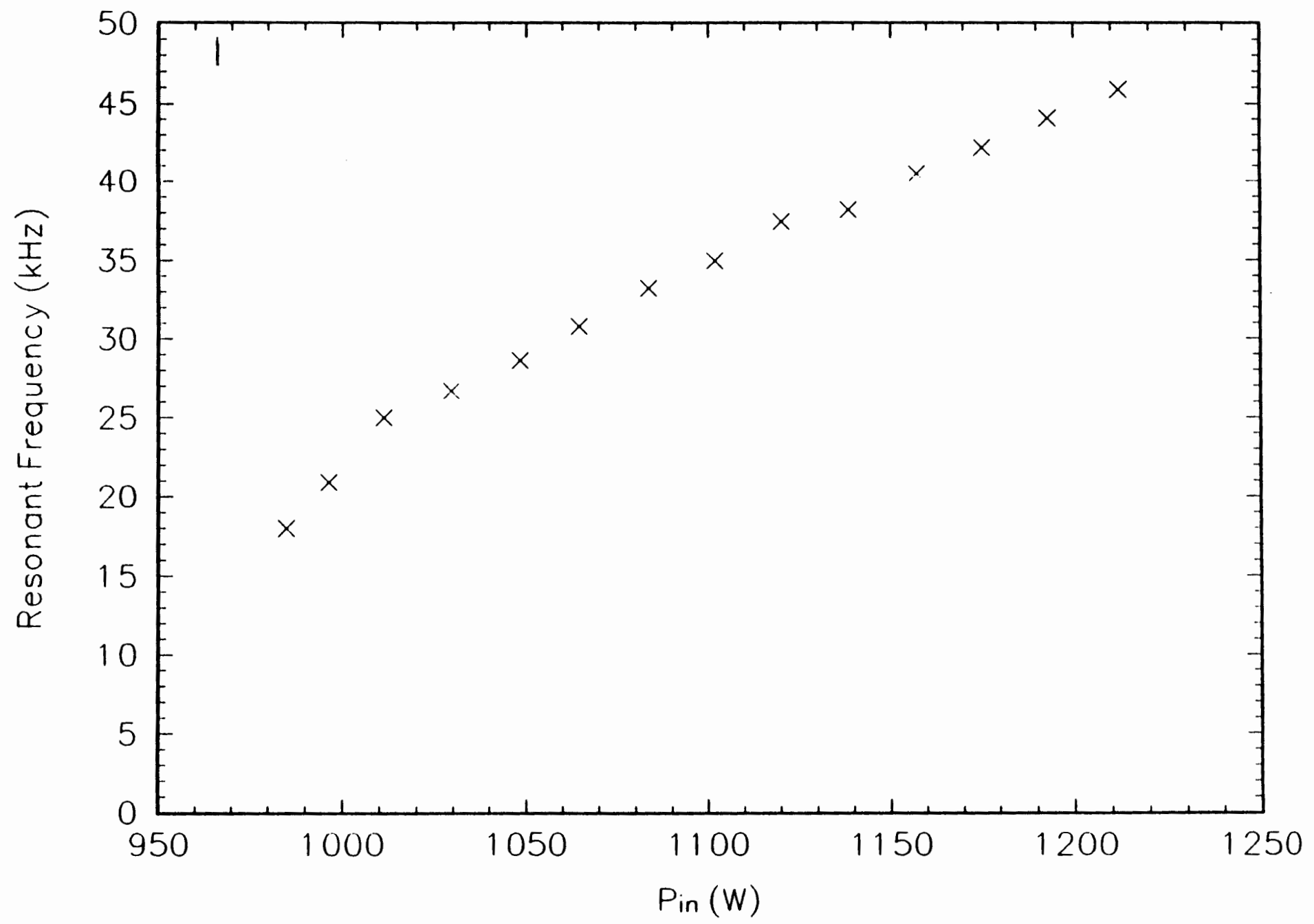

Eigure 38. The change in resonance frequency of the relaxation oscillation as a function of pump power. 


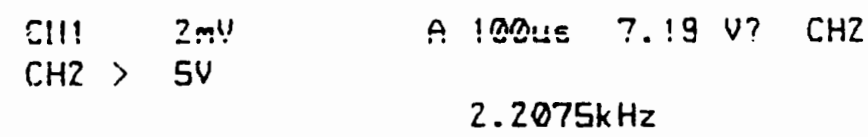

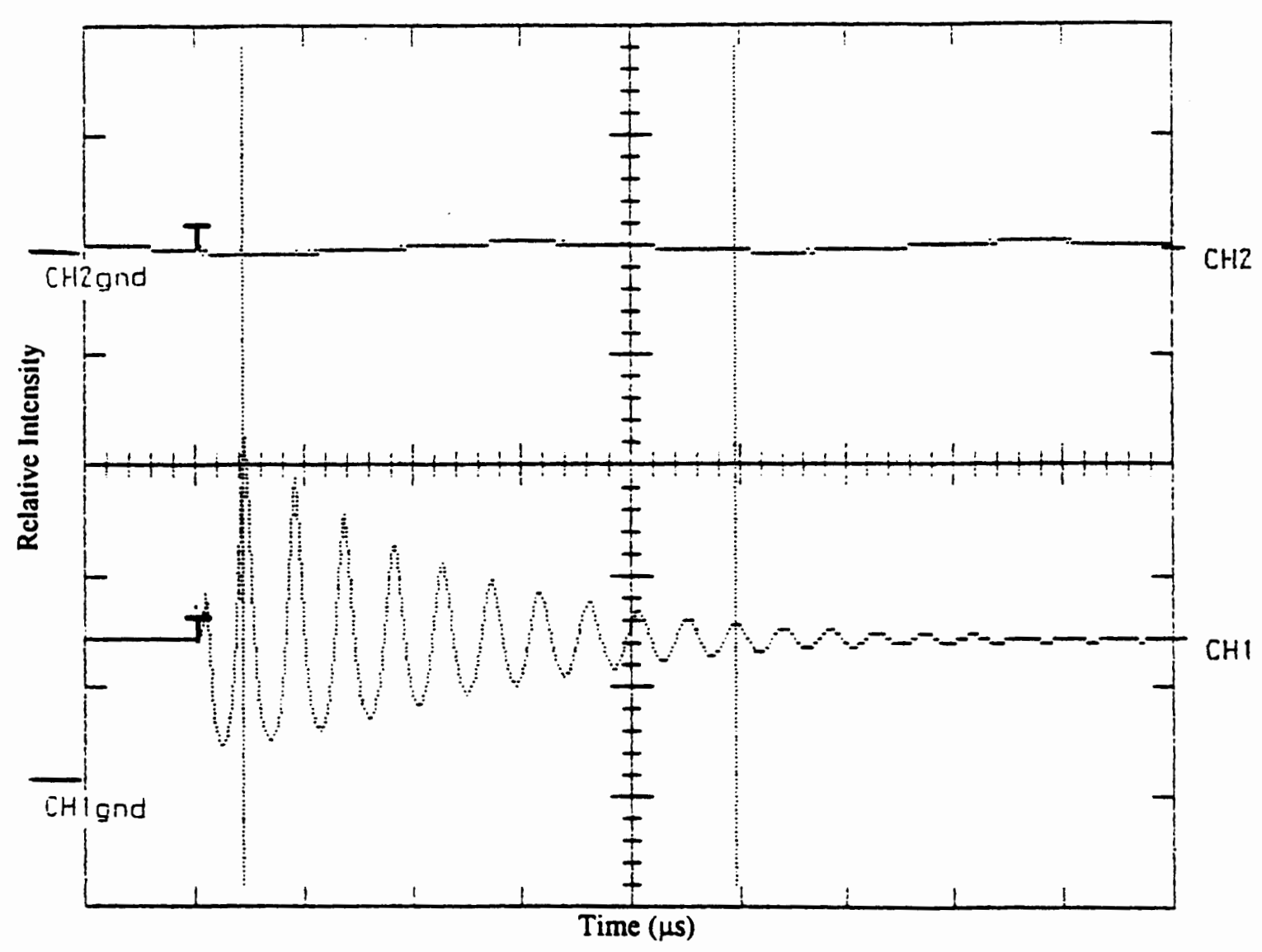

- Eiqure 39 Relaxation oscillation response of Nd:YAG laser pumped at $\mathrm{V}=85.521 \mathrm{~V}, \mathrm{I}_{\text {in }}=11.23 \mathrm{~A}, \mathrm{P}_{\text {in }}=960.38 \mathrm{~W}$; $P_{\text {out }}=7 \mathrm{~mW}, \mathrm{f}_{\mathrm{R}-0}=22.07 \mathrm{kHz}$. The display is an average of 256 frames. Note that the AOM drive signal (Ch2) averages to approximately zero due to timing jitter. 

$\begin{array}{ll}\mathrm{CHI} & 5 \mathrm{mV} \\ \mathrm{CH} 2 & >5 \mathrm{~V}\end{array}$
A 5ous 7.19 V? $\mathrm{CH} 2$
4. $3197 \mathrm{kHz}$

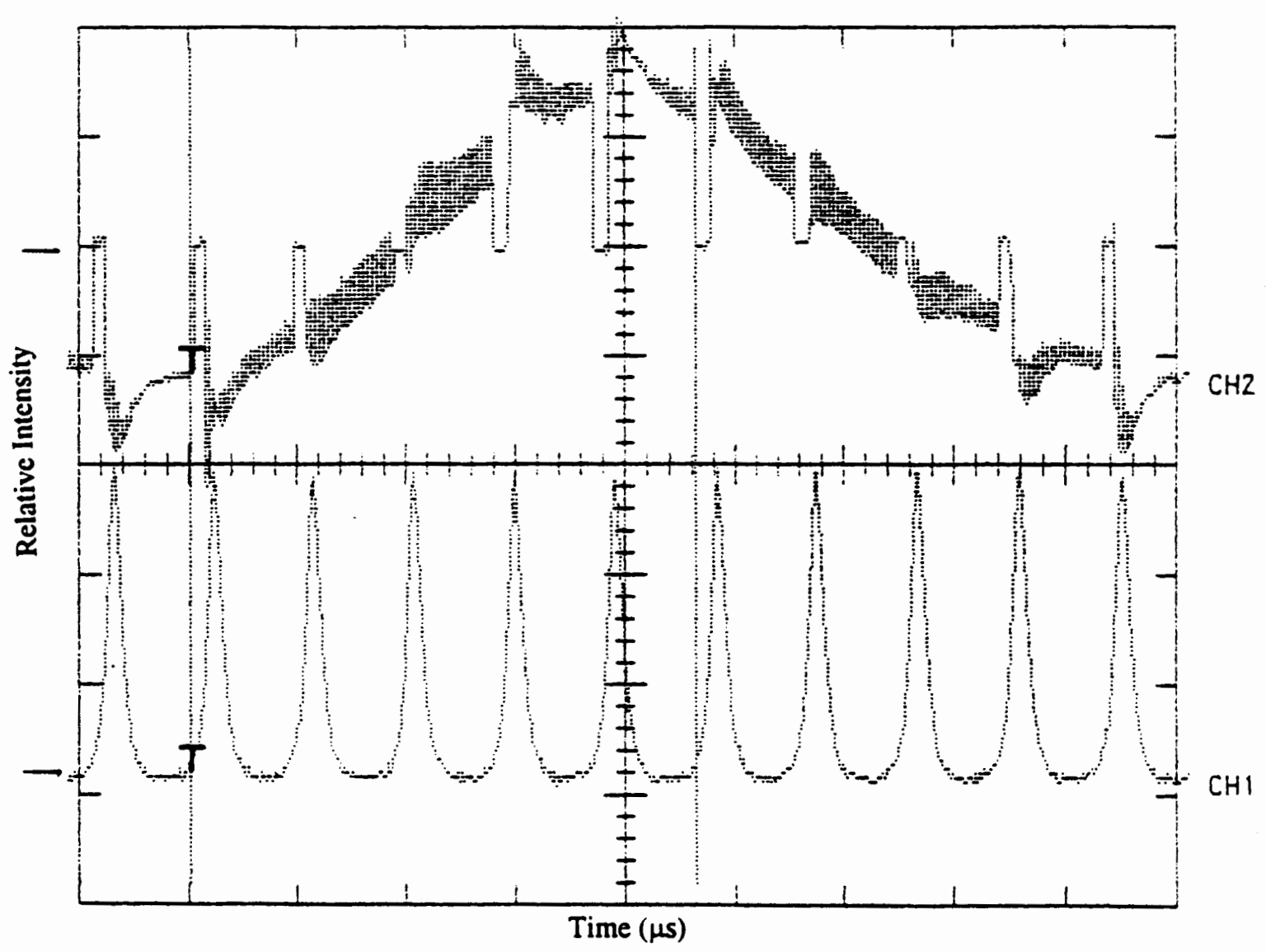

- Eigure 40 The laser response with $\mathrm{V}_{\mathrm{in}}=85.52 \mathrm{~V}, \mathrm{I}_{\mathrm{in}}=$ $11.23 \mathrm{~A}, P_{\text {in }}=960.38 \mathrm{~W} . P_{\text {out }}=7 \mathrm{~mW}$ and the modulation frequency $f_{\bmod }=21.60 \mathrm{kHz}=0.98 \mathrm{f}_{\mathrm{R}-\mathrm{O}}$. The display is not averaged. Note that the aliasing in the RF signal (Ch2). 


$$
\begin{aligned}
& \mathrm{CHI} \quad \mathrm{mV} \quad \mathrm{m} 58 \mathrm{~s} \quad 7.19 \mathrm{~V} ? \mathrm{CH} 2 \\
& 10.526 \mathrm{kHz}
\end{aligned}
$$

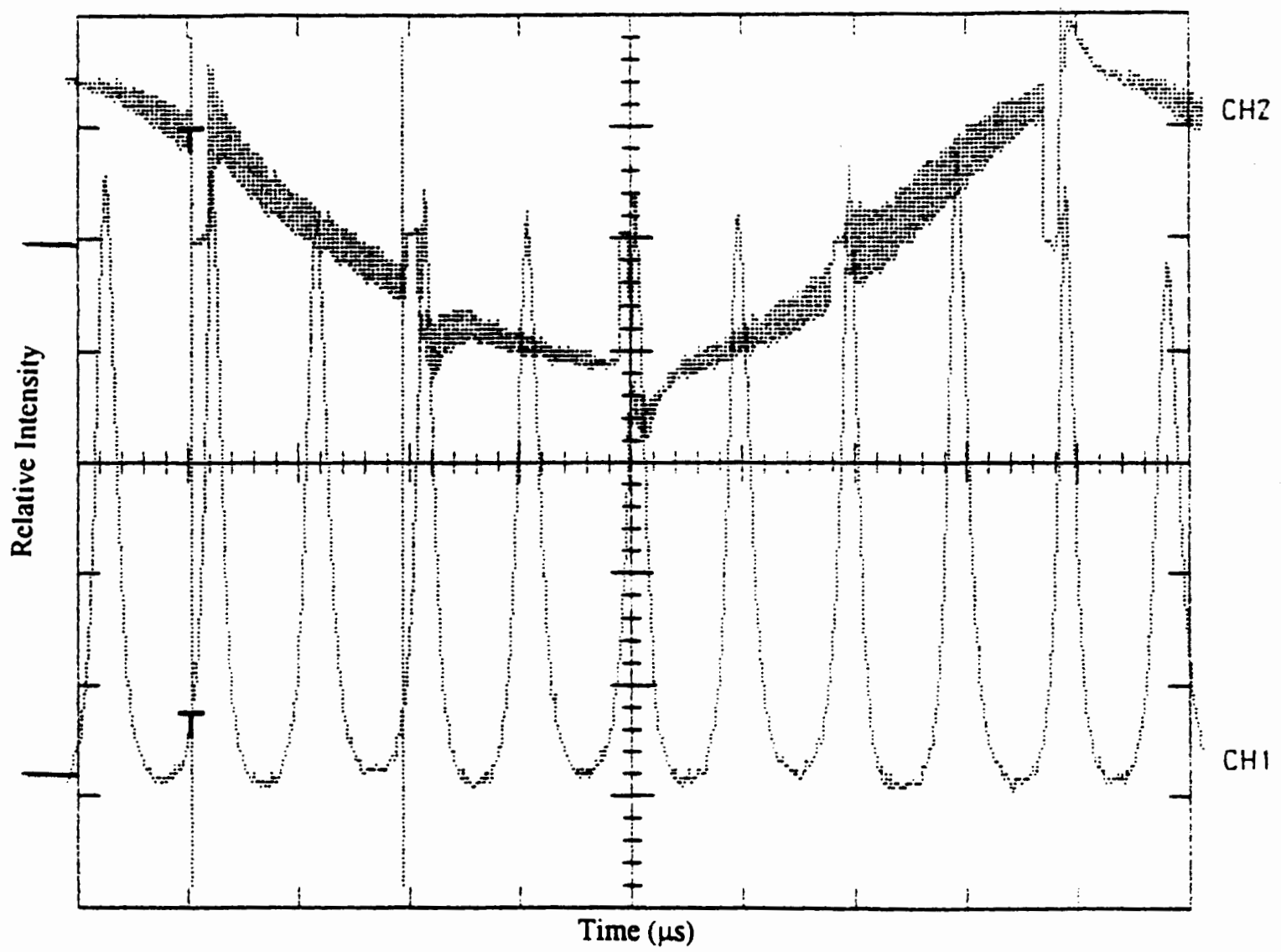

Eigure 41. The laser response with $\mathrm{V}_{\text {in }}=85.52 \mathrm{~V}, \mathrm{I}_{\text {in }}=$ $11.23 \mathrm{~A}, \mathrm{P}_{\text {in }}=960.38 \mathrm{~W} . \mathrm{P}_{\text {out }}=7 \mathrm{~mW}$ and the modulation frequency $f_{\text {mod }}=10.526 \mathrm{kHz}=(1 / 2)\left(0.99 \mathrm{f}_{\mathrm{R}-\mathrm{O}}\right)$. The display is not averaged. 
$\mathrm{CH} 1>2 \mathrm{mV}$
$\mathrm{CH} 2>5 \mathrm{~V}$

A sous $7.19 \mathrm{~V}$ ? $\mathrm{CH} 2$

$5.5096 \mathrm{kHz}$

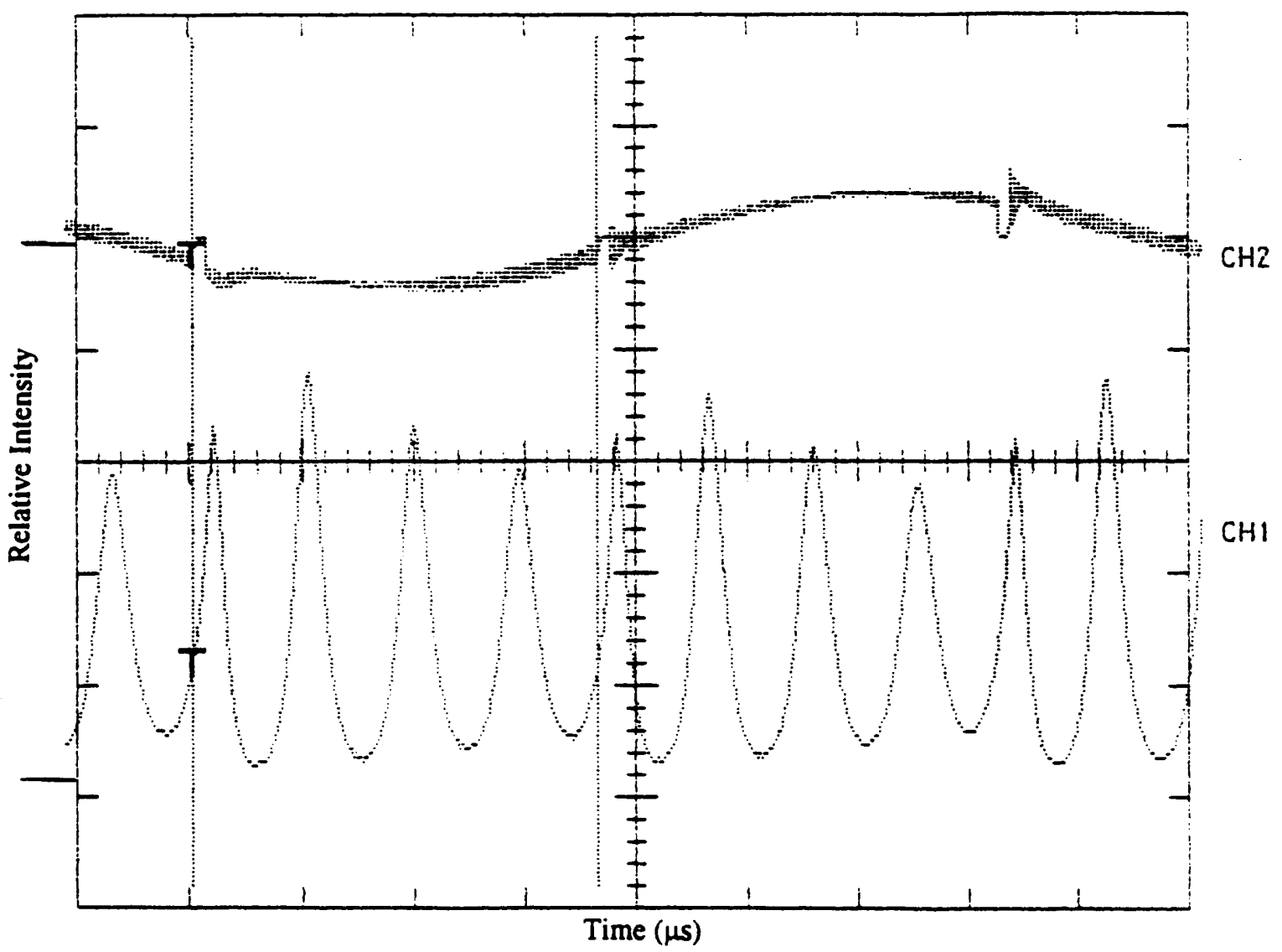

- Eigure 42. The laser response with $\mathrm{V}_{\mathrm{in}}=85.52 \mathrm{~V}, \mathrm{I}_{\mathrm{in}}=$ $11.23 \mathrm{~A}, \mathrm{P}_{\mathrm{in}}=960.38 \mathrm{~W} . \mathrm{P}_{\text {oux }}=7 \mathrm{~mW}$ and the modulation frequency $\mathrm{f}_{\bmod }=5.509 \mathrm{kHz}=(1 / 4)\left(0.99 \mathrm{f}_{\mathrm{R}-0}\right)$. The display is not averaged. 


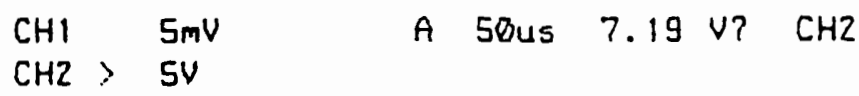

\section{$4.5455 \mathrm{kHz}$}

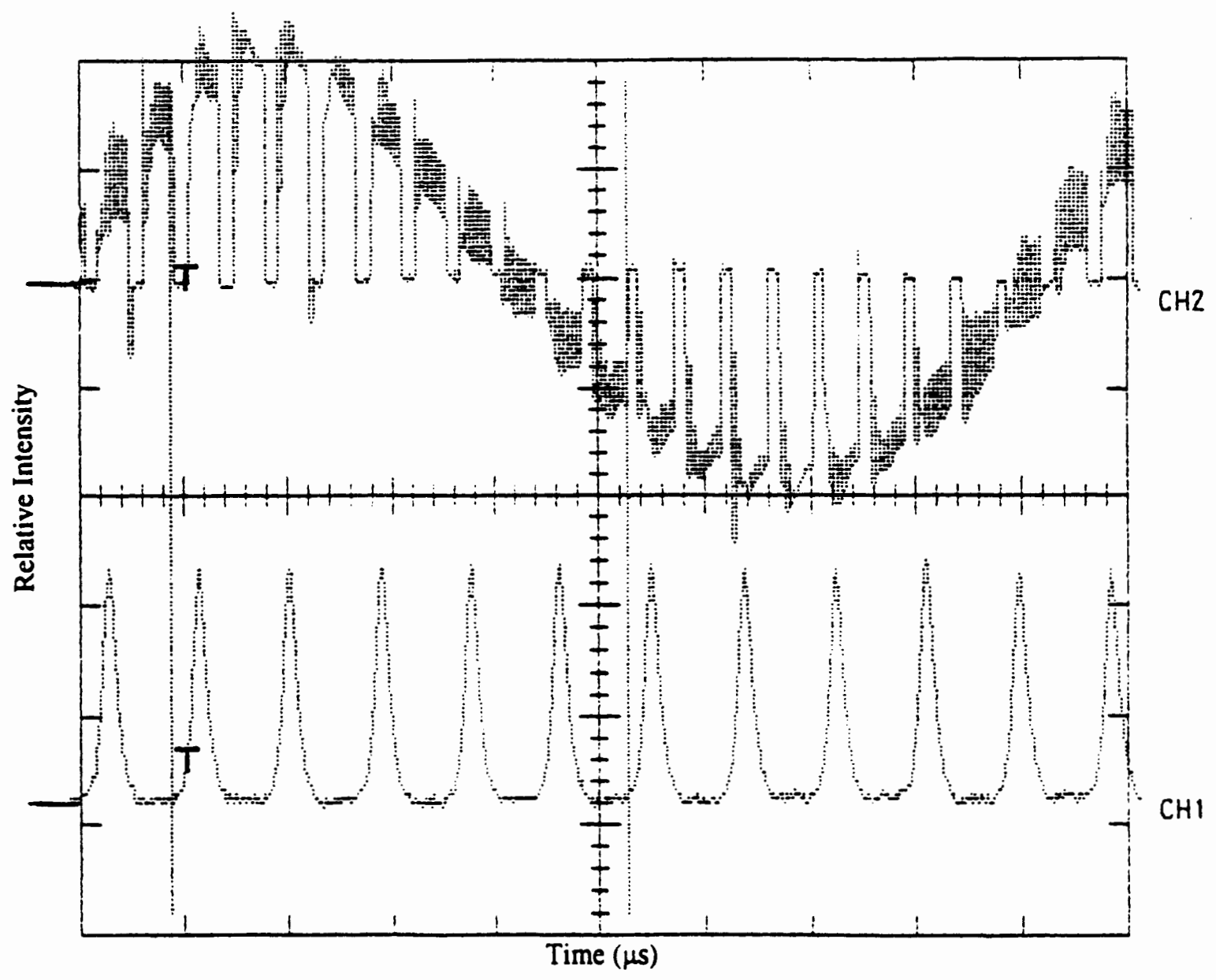

Eigure 43. The laser response with $\mathrm{V}_{\mathrm{wn}}=85.52 \mathrm{~V}, \mathrm{I}_{\mathrm{in}}=$

- 11.23 A, $P_{\text {in }}=960.38 \mathrm{~W} . \mathrm{P}_{\text {out }}=7 \mathrm{~mW}$ and the modulation frequency $f_{\text {mod }}=45.455 \mathrm{kHz}=(2)\left(1.03 \mathrm{f}_{\mathrm{R}-0}\right)$. Note that the frequency of spike train does not increase beyond $f_{\text {mod }}$ even when the modulation frequency is twice $f_{R-0}$. 


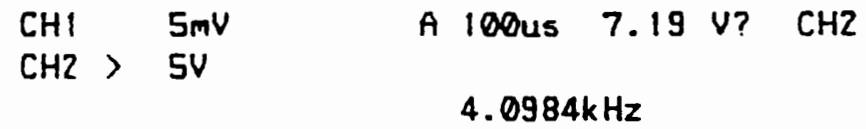

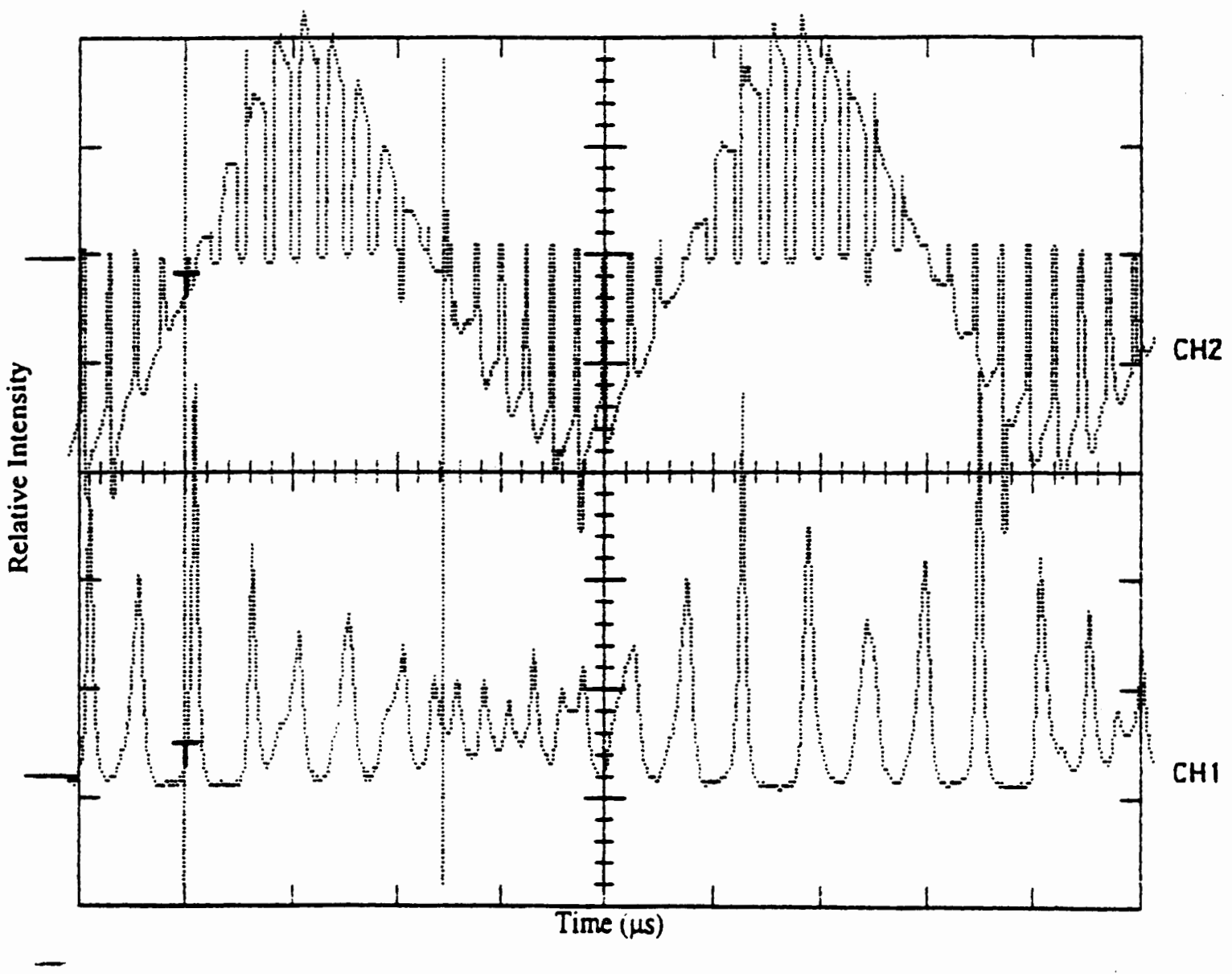

Eigure 44. The laser response with $\mathrm{V}_{\text {in }}=85.52 \mathrm{~V}, \mathrm{I}_{\text {in }}=$ $11.23 \mathrm{~A}, \mathrm{P}_{\mathrm{in}}=960.38 \mathrm{~W}$. Note that the modulated frequency is not a multiple or submultiple of resonance frequency. 


\section{CHAPTER IV}

\section{DISCUSSION}

In order to compare the experimental and the theoretical results, the experimental values of the pump power need to be multiplied by the proportionality factor $k_{3}$ determined in Appendix B so that it is possible to estimate the amount of power the lamp emits in the $808 \mathrm{~nm}$ region. In addition, let us normalize the pump power, for both experimental and the theoretical case, by subtracting from the pump power the threshold pump value. The last step makes it possible to compare the experimental and the theoretical resonant frequency as a function of pump power on the same graph.

The results from experiments and the computer simulations both show that the resonance frequency of the relaxation-oscillation increases with increasing pump power (Figure 45). The increase in the resonance frequency with increasing pump power is expected because with higher pump power, the gain of the laser medium increases -- implying that the incremental change in intensity with distance (i.e. $\Delta \mathrm{V} / \Delta \mathrm{z}$ ) also increases. But the cavity transit time is constant and is independent of the pump power. Therefore $\Delta \mathrm{V} \Delta \mathrm{t}$, which is the product of $\Delta \mathrm{V} / \Delta \mathrm{z}$ and 'c,' the speed of light, also increases. This means that after a perturbation is introduced into the laser, the circulating intensity, I, will return to equilibrium faster, via higher oscillation frequency, at higher pump powers than at lower pump powers. This effect is apparent if one compares Figures 12B and 17B in the case of the theoretical analysis, or Figures 26 and 36 in the case of the experimental analysis. In Fig. 12B, the laser, pumped at $225 \mathrm{~mW}$, takes approximately $800 \mu \mathrm{s}$ to return 
to the original steady state output after a perturbation is introduced. In Fig 17B, the laser pumped at $500 \mathrm{~mW}$, takes less than $400 \mu \mathrm{s}$ to return to the original steady state value after a similar perturbation.

While the results from the experiments and the computer simulations show similar response to a perturbation, there is a discrepancy in the absolute numerical values for the two cases. One key factor contribution to the discrepancy is the fact that the program assumes pumping at $808 \mathrm{~nm}$ only and ignores all other absorption lines (Fig. 2) while the CVI laser used in the experiment is pumped with a flash lamp that emits in a broad spectrum. To simulate the operation of the laser used in this experiment, the rate equations have to be rewritten to take into account of absorption at these other lines. The pump term $P$ in equation (4), instead of being a simple numerical value, should represent the convolution of the absorption spectrum of $\mathrm{Nd:YAG}$ and the black-body spectrum of the lamp for a given filament voltage and current. Both the voltage and the current must be used since the filament resistance has a strong non-linear temperature dependence. If the value of $\mathrm{P}_{\mathrm{N}}$ determined in Appendix $\mathrm{B}$ using experimental data is larger than pump values used in the basic program, then this difference can also contribute to the above discrepancy since $P_{N^{3}}$ is a lumped parameter, which includes contributions from all pump wavelength not just $808 \mathrm{~nm}$. Other factors leading to the discrepancy are the uncertainties such as whether or not the laser is oscillating in the $\mathrm{TEM}_{\infty}$ mode, and the accuracy of measured values of parameters like the beam waist which is used to determine the beam area. An accurate determination of the beam or mode area is important since this parameter is used extensively in the present work to determine many other parameters from the mode volume to the proportionality factor $\mathrm{k}_{3}$ (Appendix B).

When attempting to drive the laser at sub- or multiple harmonic frequencies, it was observed that a train of spikes could be obtained, both in the theoretical and experimental analysis, even when the modulation frequency was different from the exact resonant re- 
laxation oscillation frequency by as much as $9 \%$ (Fig. 18-22, 40-43). However, for each sub- or multiple harmonic case, there is only one unique frequency which will result in a spike train having a maximum amplitude. The ability to induce resonant or spike train behavior by modulation at or near sub- or multiple harmonic frequencies is possible due to the nonlinearities present in the Nd:YAG laser. Since the modulation frequency is a function of intensity in a nonlinear system, the successive periods of relaxation oscillation wave forms change slightly with time. The phenomenon that the spike train behavior can be induced over a range of frequencies can be thought of, to some extent, as being similar to the mode pulling phenomenon observed in many lasers. 


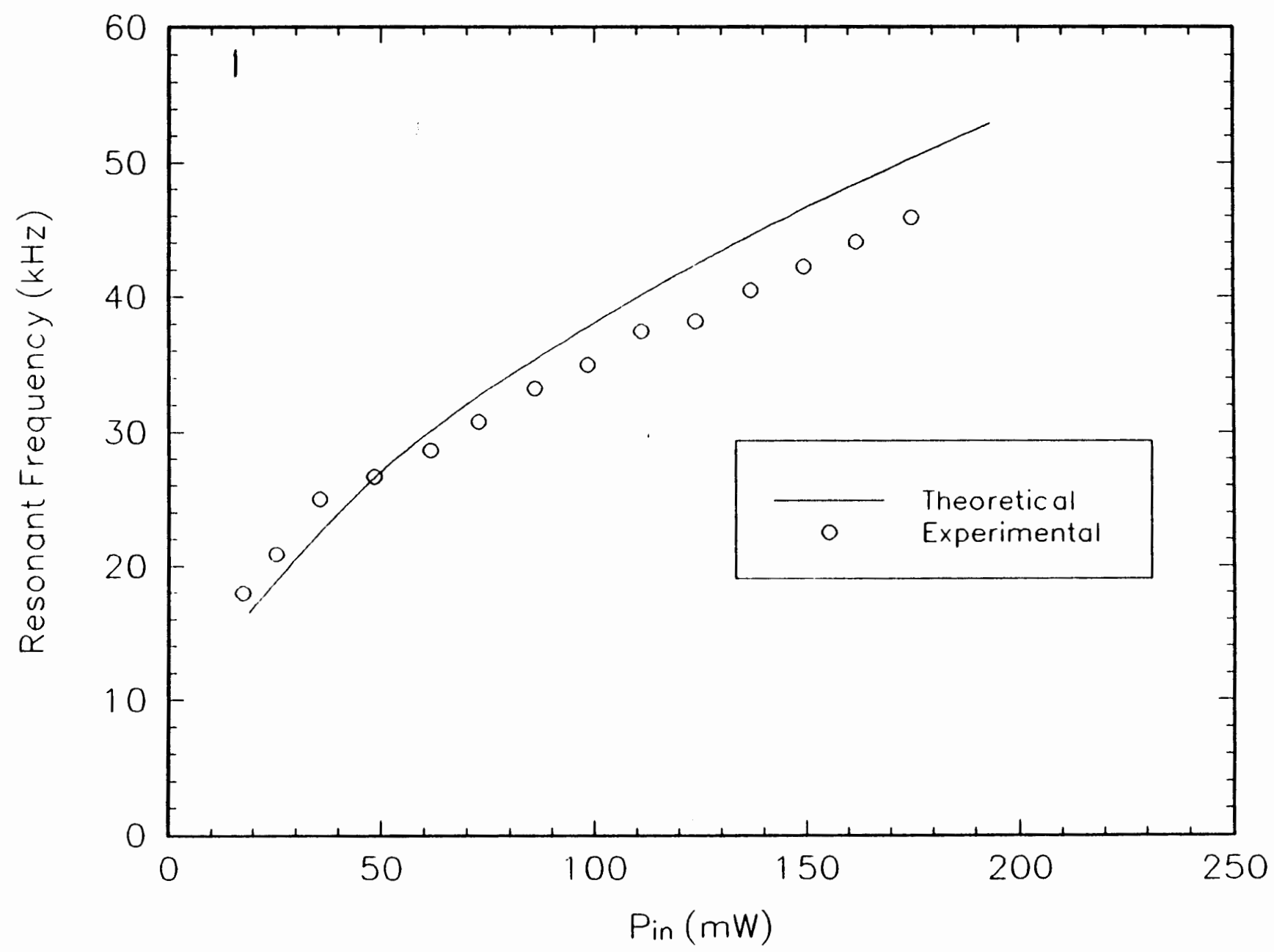

Eigure 45. Comparison of the experimental and the theoretical relaxation oscillation values as a function of pump power. 


\section{CHAPTER V}

\section{CONCLUSION}

In the preceeding chapters, nonlinear rate equations, including cavity perturbation term, were solved numerically by a Runga-Kutta method using experimentally measured parameter values to study the relaxation oscillation and the spiking behavior in a Nd:YAG laser. The equations were solved for a general four-level case while minimizing the number of assumptions made and include terms representing the steady state (un-pumped) equilibrium populations for each level due to the Boltzmann distribution.

It was shown analytically, for the case of a cw pumped Nd:YAG laser, that when the cavity gain or loss was perturbed, the output intensity experienced an amplitude modulation or became a regular train of spikes, with the frequency depending on the pump power. In the case of loss modulation, it was shown that by modulating the cavity loss at or near the sub-harmonics of the resonant frequency, one could still obtain the original spike train pattern but with additional components (although smaller in amplitude) located at time intervals equal to the incerse of the driving frequency.

- A flashlamp pumped Nd:YAG laser was used to verify the theoretical results. By attenuating the $R F$ signal supplied to the intracvity $A O M$, it was possible to introduce perturbations in the laser in the form of loss modulation. The experimental results show that the resonant frequency of relaxation oscillation is dependent on the pump power, cavity parameters like cavity loss, and parameters inherent to the gain medium such as the flourescent lifetimes. 
Both the theoretical and the experimental results are consistant with the theory proposed by McCumber. The model used in the present work is useful if pumping at only the $808 \mathrm{~nm}$ band. To simulate pumping using a flashlamp, the pump term $\mathrm{P}$ in Eq. (4) should represent the convolution of the absorption spectrum of Nd:YAG and the broad band black-body emission spectrum of the flashlamp. 


\section{REFERENCES}

[1] D. E. McCumber, "Intensity fluctuations in the output of cw laser oscillator, I," Phys. Rev., vol. 141, pp. 306-322, January 1966.

[2] J. E. Geusic, H. M. Marcos, and L. G. Van Uitert, "A study of Nd:YAG laser," in Physics of Quantum Electronics, P. L. Kelly, B. Lax, and P. E. Tannenwald, Eds. New York: McGraw-Hill, 1966, pp. 725-734.

[3] T. Ikegami and Y. Suematsu, "Resonance-like characterisitic of the direct modulation of a junction laser," Proc. IEEE (Letters), vol. 55, pp. 122-123, January 1967.

[4] T. Kimura and $\mathrm{K}$. Otsuka, "Response of a $\mathrm{cw} \mathrm{Nd} \mathrm{d}^{+3}: \mathrm{YAG}$ laser to sinusoidal cavity perturbations," IEEE J. Quantum Electron., vol. QE-6, pp. 764-769, December 1970.

[5] W. Koechner, Solid State Laser Engineering. New York: Springer-Verlag, 1992.

[6] A. A. Kaminskii, Laser Crystals. New York: Springer-Verlag, 1981.

[7] J. Verdeyen, Laser Electronics. Englewood Cliffs: Prentice-Hall, 1989.

[8] F. E. Hovis, M. Stuff, C. J. Kennedy, and B. Vivian, "Lower level relaxation of Nd:YAG," IEEE J. Quantum Electron., vol. QE-28, pp. 39-42, January 1992.

[9] A. A. Demokan, Mode Locking in Solid-State and Semiconductor Lasers. New York: Research Studies Press, 1982.

[10] J. Cruz, G. Giuliani, and H. M. van Driel, "Measurement of the subnanosecond, nonradiative relaxation time from excited states of $\mathrm{Nd}^{+3}$ in a $\mathrm{Nd}$ : $\mathrm{YAG}$ crystal," Optics Letters, vol. 15, pp. 282-284, March 1990.

[11] A. Seigman, Lasers. Mill Valley: University Science Books, 1983. 


\section{APPENDIX A}

CALCULATION OF BEAM DIAMETER 
The spot size of the beam, $2 w_{0}$, exiting the laser was determined using a CCD camera (Model EDC-1000HR Computer Camera, Electrim Corp., Princeton, NJ) to look at the beam reflecting off of a "speckle remover" located $183 \mathrm{~cm}$ from the output coupler (OC) of the laser. A speckle remover, a piece of rotating white poster board, is used to minimize erroneous intensity measurements resulting from laser speckle effects. The speckle pattern changes as the disc is rotated, due to the slight surface abnormalities on the face of the disc. A large camera integration time was chosen so that the camera responds to the average reflected intensity.

The output from the camera is a 2-D image which is stored in a buffer file consisting of a one dimensional array of 32,340 bytes corresponding to 165 lines of 196 bytes each. The 192 bytes of each line correspond to a row of 192 pixels. Each CCD element has eight bits of dynamic range, which correspond to 256 grey levels. A pixel value of 1 represents black while a pixel value of 255 represents white.

Before making any measurements, an image of a calibrated ruler, located at the same position as the speckle remover, was captured to obtain the horizontal scaling factor that was used when displaying subsequent beam profiles. To evaluate the beam profile, a cross section through the center of the image was obtained and displayed on a monitor using software supplied by the camera manufacturer. Figure A1 depicts a profile of the beam from the laser used in the current experiment. By taking the ratio of the diameter of the profile at the $1 / \mathrm{e}^{2}$ point from the peak intensity (i.e. $2.2 \mathrm{~cm}$ in Figure $\mathrm{A} 1$ ) to the absolute-distance corresponding to $1 \mathrm{~cm}$ of horizontal axis (i.e. $6.4 \mathrm{~cm}$ ), the $1 / \mathrm{e}^{2}$ beam diameter was determined to be $3.4375 \mathrm{~mm}$. Since the measurement was taken approximately 183 $\mathrm{cm}$ from the output coupler, the value of the spot size at the output coupler had to be back-calculated from the Gaussian beam propagation equation

$$
w(z)=w_{0}^{2}\left[1+\left(z / z_{0}\right)^{2}\right]^{1 / 2}
$$


where $w(z)$ is the beam radius measured a distance $z$ from the output coupler, and $z_{0}$ is the Rayleigh range of the laser, defined by $z_{0}=\pi w_{0}^{2} \lambda$. To determine the beam waist $w_{0}$, substitute into (A1) an estimated value of $w_{0}$, calculate $w(\mathrm{z})$, compare with the measured $w(z)=1.7188 \mathrm{~mm}$, and iterate until a value of $w_{0}$ is found such that $w(z)_{\text {calc }} \sim w(z)_{\text {exp. }}$ The results of this exercise are given in Table A1.

TABLE A1

DETERMINATION OF $w(z)$ USING THE ITERATIVE METHOD

$\begin{array}{ll}w_{\mathrm{o}}(\mathrm{mm}) & w(z)(\mathrm{mm}) \\ & \\ 0.3000 & 2.086 \\ 0.3500 & 1.803 \\ 0.3650 & 1.736 \\ 0.3675 & 1.714 \\ 0.3750 & 1.694 \\ 0.4000 & 1.599\end{array}$

The beam waist was determined to be approximately $0.3675 \mathrm{~mm}$ which corresponds to a Rayleigh range of approximately $40 \mathrm{~cm}$. Since the laser rod length of $6 \mathrm{~cm}$ is less than the Rayleigh length, one can assume a uniform intensity across the beam and therefore calculate the mode area using the formula for the area of a circle. The mode area and the mode volume were determined to be $0.0042429 \mathrm{~cm}^{2}$ and $0.0254574 \mathrm{~cm}^{3}$, respectively.

Because of the significance of the mode area and mode volume values used in the basic program to determine the threshold pump power for lasing, and also the steady state output power, care was taken to ensure that there was no interference from the camera front window and, more importantly, that the camera pixels did not saturate (since the CCD camera is designed to work with low powers like 1-2 mW.) To eliminate the risk of 
saturation, the aperture on the camera lens was adjusted until it was barely open. Additionally, neutral density filters were placed between the $\mathrm{OC}$ and the speckle remover. One other improvement (although not possible in the present study), which would further result in more accurate value of the beam waist, is to take an average over a large number (like ten) scans. 


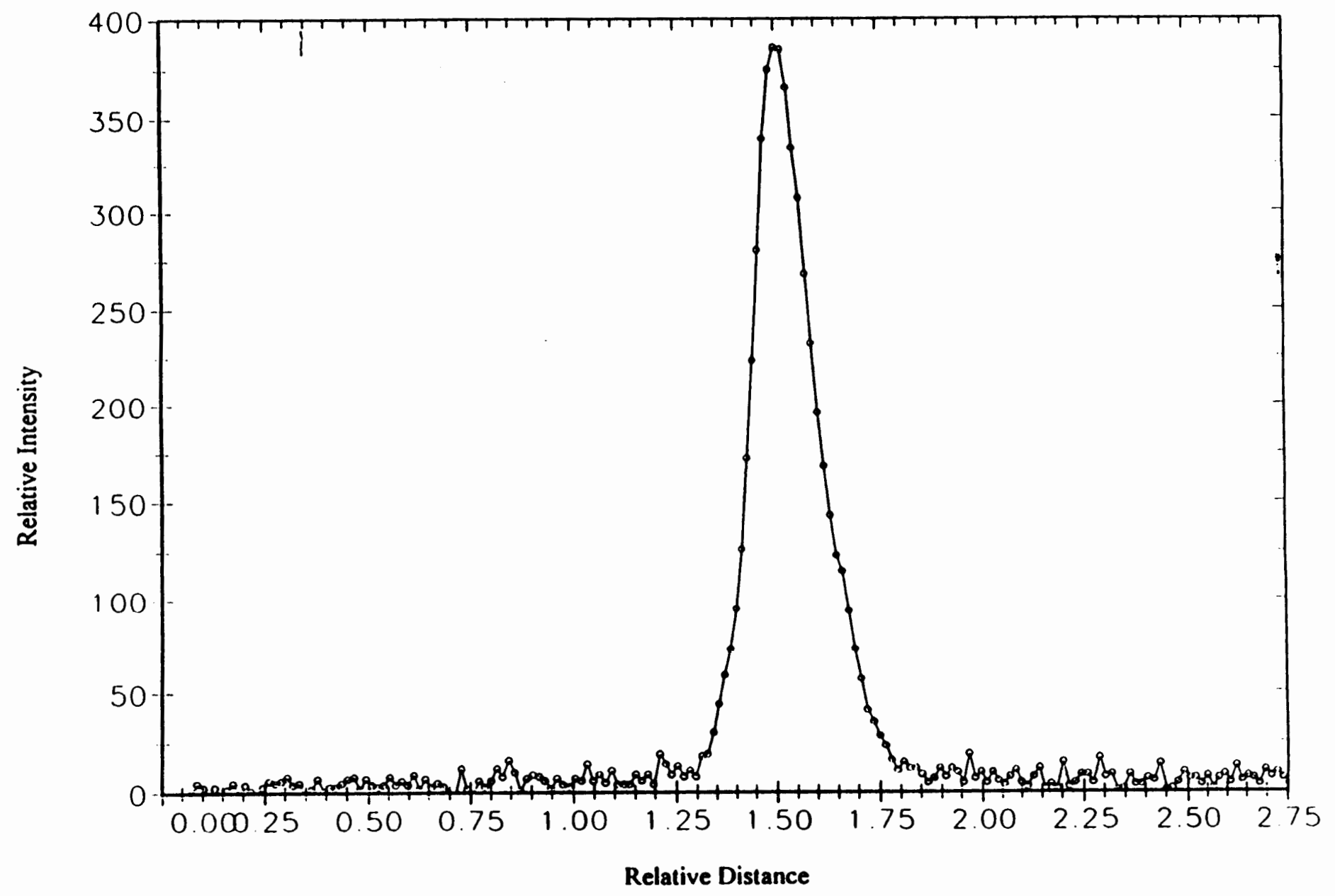

Figure A1. The beam profile of the output from the CVI C-95 Nd:YAG laser. 


\section{APPENDIX B}

THE DETERMINATION OF THE DISTRIBUTED CAVITY LOSSES $\alpha_{\mathrm{T}}$ AND THE GAIN $\gamma_{0}$ PER ROUND TRIP 
In this appendix, the distributed cavity losses $\alpha_{\mathrm{T}}$ and the gain $\gamma_{\mathrm{o}}$ per round trip are determined from experimental data. In addition, the ratio of the cavity loss when the acousto-optic modulator (AOM) is not driven (i.e. $\alpha_{\mathrm{T}}$ low-loss case) to the cavity loss when the AOM is supplied with RF power (i.e. $e_{\mathrm{T}}$, high-loss case) is also determined. This ratio, the contribution of the losses from the AOM is also determined.

\section{Determination of $\alpha_{\text {int }}$ and $\gamma_{0}$}

The condition of steady state output in a laser is reached when the saturated gain exactly balances the losses, which have been prorated over the length of the gain medium. In the Nd:YAG laser, it is safe to assume that the laser transition is homogeneously broadened and that the laser oscillates close to line center, so that the frequency dependence of the saturation behavior and gain can be ignored.

To determine the gain and losses in the laser, we begin with the equation for steady state operation given in [7].

$$
I_{\text {out }}=\frac{I_{3}}{2}\left(\frac{\gamma_{0}}{\alpha_{T}}-1\right)\left(T_{1}+T_{2}\right)
$$

where $I_{s}$ is the saturation intensity

$\gamma_{0}$ is the gain coefficient per unit length in the inverted gain media

$\alpha_{\mathrm{T}}$ is the distributed losses in the laser

$T_{1}, T_{2}$ are the mirror transmission coefficients of the resonator mirrors $M_{1}, M_{2}$.

The term Is is a constant with the dimension of (Watts/unit area) and is defined by

$$
I_{s}=\frac{h v}{\tau_{10}+\tau_{2}-\tau_{1} \tau_{2} / \tau_{21}} \cdot \frac{1}{\sigma_{21}} .
$$

Using the numerical values of $\tau$ 's, $\sigma_{21}$, and $h v$ from Table $1, I_{s}$ is calculated to be $I_{s}=$ $1249.617 \mathrm{~W} / \mathrm{cm}^{2}$.

Typically, the back mirror can be thought of as a $100 \%$ reflector and therefore $\mathrm{T}_{2}$ can be set equal to zero. The term $\alpha_{\mathrm{T}}$ can be expressed as a sum of two types of losses: 
those which are internal to the laser such as scattering, diffraction, reflection, absorption in the mirrors, the amplifying medium, and all other elements in the resonator; and those which represent coupling to the outside of the laser, namely, the mirror reflectivities. In equation form

$$
\alpha_{\mathrm{T}}=\alpha_{\mathrm{int}}+\alpha_{\mathrm{ext}}=\alpha_{\mathrm{int}}+\frac{1}{2 l} \ln \frac{1}{\mathrm{R}_{1} \mathrm{R}_{2}}
$$

where $l$ is the physical length of the gain medium.

$R_{1}$ and $R_{2}$ represent the reflectivities of mirrors $M_{1}$ and $M_{2}$, respectively. Again $R_{2}$ can be set equal to 1 for same reason $T_{2}$ was eliminated in (B1).

Solving (B1) for $\gamma_{\alpha} / \alpha_{1}$, and renaming $R_{1}$ as $R_{o c}$, the reflectivity of the output coupler, one gets

$$
\frac{\gamma_{0}}{\alpha_{T}}=1+\frac{I_{0}}{I_{s}} \cdot \frac{2}{1-R_{\infty}}
$$

where the transmission of the output coupler, $T$, has been replaced by $1-R_{o c}$ To simplify (B3), the term $A\left(R_{o c}\right)$ is introduced to represent the right hand side of (B3). The unknowns in (B3) are $\gamma_{0}$ and $\alpha_{\text {int }}$ which is imbedded in $\alpha_{\mathrm{r}}$. In order to solve for the two unknowns we need another equation. One simple way to generate another equation is to write $\gamma_{0}$ and $\alpha_{\mathrm{T}}$ as function of the output couple reflectivity:

$$
\frac{\gamma_{0}\left(R_{o c} l\right)}{\alpha_{\text {int }}+\frac{1}{2 l} \ln \frac{1}{R_{o c} l}}=1+\frac{I_{0}\left(R_{o c} 1\right)}{L} \cdot \frac{2}{1-R_{o c} 1}=A\left(R_{o c} 1\right) .
$$

By substituting (B2) for $\alpha_{\mathrm{T}}$ in (B3) and rewriting (B3) for two different mirror reflectivities, $R_{o c} 1$ and $R_{o c} 2$, the result is

$$
\frac{\gamma_{0}\left(R_{o c} 2\right)}{\alpha_{\mathrm{vt}}+\frac{1}{2 l} \ln \frac{1}{R_{\propto c} 2}}=1+\frac{I_{0}\left(R_{o c} 2\right)}{I_{s}} \cdot \frac{2}{1-R_{o c} 2}=A\left(R_{o c} 2\right)
$$


The above equations can again be rewritten in the form of an equation for a straight line

$$
\begin{aligned}
& \gamma_{0}\left(R_{\propto c} 1\right)=\alpha_{\text {int }} A\left(R_{\propto c} 1\right)+\frac{A\left(R_{o c} 1\right)}{2 l} \ln \left(\frac{1}{R_{o c} 1}\right) \\
& \gamma_{0}\left(R_{\propto c} 2\right)=\alpha_{\text {int }} A\left(R_{\propto c} 2\right)+\frac{A\left(R_{o c} 2\right)}{2 l} \ln \left(\frac{1}{R_{\propto c} 2}\right) .
\end{aligned}
$$

Subtracting (B5) from (B4) results in,

$$
\begin{aligned}
& \gamma_{0}\left(R_{\circ c} 1\right)-\gamma_{0}\left(R_{\circ c} 2\right) \\
& =\frac{A\left(R_{\propto c} 1\right)}{21} \ln \left(\frac{1}{R_{\propto c} 1}\right)-\frac{A\left(R_{\propto} 2\right)}{21} \ln \left(\frac{1}{R_{\propto c} 2}\right)+\alpha_{\text {int }}\left[A\left(R_{\propto 1} 1\right)-A\left(R_{\propto c} 2\right)\right] .
\end{aligned}
$$

If the pumping of the laser rod while out coupling with reflector $R_{o c} 1$ is the same as when out coupling with reflector $R_{o c} 2$ (i.e. $P_{p}\left(R_{o c} 1\right)=P_{p}\left(R_{o c} 2\right)$ ), then the gain in both cases is the same (i.e. $\gamma_{o}\left(R_{o c} 1\right)=\gamma_{0}\left(R_{o c} 2\right)$ ). Consequently, the left side of (B6) can be set to zero and one can solve the resulting equation for $\alpha_{\text {int }}$ to get

$$
\alpha_{\text {int }}=\left[\frac{A\left(R_{\propto} 2\right)}{2 l} \ln \left(\frac{1}{R_{\propto c} 2}\right)-\frac{A\left(R_{\propto} 1\right)}{21} \ln \left(\frac{1}{R_{\propto c} 1}\right)\right]\left[A\left(R_{\propto c} 1\right)-A\left(R_{\propto c} 2\right)\right]^{-1} .
$$

By measuring the output power of the laser for same pump power levels but with two different output couplers, (B7) enables one to determine $\alpha_{\text {int. }}$ The values of $I_{0}$ can be obtained by dividing the output power by the mode area determined in Appendix A. Having found $\alpha_{\text {int }}$, one can easily calculate the value of the gain coefficient $\gamma_{0}$ by substituting the value of $\alpha_{\text {int }}$ into (B4) or (B5).

To obtain a more accurate value of $\alpha_{\text {int }}$ and $\gamma_{0}$, the output power was measured over a range of input powers, and the results were averaged. A simple Mathcad program (CVIGAIN.MCD) was written to carry out the iterative calculations. Table B1 contains the input and output powers using two different output coupler mirrors. 
TABLE B 1

OUTPUT POWER AS A FUNCTION OF MIRROR REFLECTIVITY

AND PUMP POWER

\begin{tabular}{llll} 
& $\mathrm{R}_{\mathrm{oc}} 1$ & \multicolumn{2}{c}{$\mathrm{R}_{\mathrm{oc}} 2$} \\
$\mathrm{P}_{\text {in }}(\mathrm{W})$ & $\mathrm{P}_{\text {oul }}(\mathrm{mW})$ & $\mathrm{P}_{\text {in }}(\mathrm{W})$ & $\mathrm{P}_{\text {out }}(\mathrm{mW})$ \\
998.58 & 79.5 & 1005 & 6 \\
1017.90 & 87.5 & 1014.82 & 10.75 \\
1052.82 & 100 & 1049.41 & 37 \\
1088.62 & 112.5 & 1085.61 & 67.5 \\
1124.98 & 127.5 & 1125.35 & 99.5 \\
1160.56 & 145 & 1161.46 & 132.5 \\
1199.00 & 162.5 & 1200.79 & 172.5
\end{tabular}

Because of the instability of the laser output when pumping near threshold in the case using $R_{o c} 2$, the value of $\alpha_{i n t}$ for this case was not used in the average. The values of $\gamma_{0}$ and $\alpha_{\text {int }}$ were determined to be $0.006901 \mathrm{~cm}^{-1}$ and $0.0004497 \mathrm{~cm}^{-1}$, respectively.

Using the value of $\alpha_{\text {int }}=0.0004497 \mathrm{~cm}^{-1}$ in (B2), $\alpha_{\mathrm{T}}$ is found to be $0.001346 \mathrm{~cm}^{-1}$. Knowing $\alpha_{\mathrm{T}}$, one can calculate the distributed round trip loss $\left(\mathrm{RT}_{\text {loss }}\right)$ over the length of the laser rod using the simple equation

$$
\begin{aligned}
& \mathrm{RT}_{\text {loss }}=21 \alpha_{\mathrm{T}} \\
& \mathrm{RT}_{\text {loss }}=1.615 \% .
\end{aligned}
$$

-.. The value of $\alpha_{\mathrm{T}}=0.001346 \mathrm{~cm}^{-1}$ represents the round trip loss under low loss conditions since the AOM in the cavity acts as a passive element when it is not supplied with any RF power. Calculation of the round trip loss when the AOM acts as an active device is taken up in the next section. 
Determination of the high and low loss values.

The acousto-optics Q-switch, under normal use, works by degrading the quality factor $Q$ of the laser during the pumping so that the gain can build up to a very high value and yet not exceed the oscillation threshold value. When the population inversion in the gain medium reaches its peak, the $Q$ is restored abruptly to its high value. The gain, which is well above the (lowered) oscillation threshold, causes a rapid build up of the oscillation field and a simultaneous depletion of the population inversion via stimulated emission. The objective of this section is to calculate the ratio of the cavity losses when the AOM acts as a passive element (low loss case, Case 1) and when the AOM acts an active element (high loss case, Case 2).

We begin with a mathematical expression for the saturated gain coefficient for a generalized model of the two atomic states involved in the laser as shown in Figure B1,

$$
\gamma=\frac{\left[R_{2} \tau_{2}\left(1-\tau_{10} / \tau_{21}\right)-R_{1} \tau_{10}\right] \sigma_{21}}{1+\left(\tau_{10}+\tau_{2}-\tau_{10} \tau_{2} / \tau_{21}\right)\left(\sigma_{21} I_{21} / h v\right)} .
$$

The R's represent rate of population increase for a given level due to direct pumping from ground state and any other indirect routes, such as excitation to and subsequent spontaneous emission from a higher state, but not those routes indicated in the diagram. The effective decay from level two is represented by $\tau_{2}$ as defined in Table 1 . Since both $R_{1}$ and $\tau_{10}$ are small numbers with respect to the first term in the bracket in the numerator, the product of $\left(R_{1} \tau_{10}\right)$ is even a smaller number and therefore can be eliminated from (B9).

Near threshold or in the limit $I_{21} \rightarrow 0$, the denominator in (B9) approaches unity and one obtains the small-signal gain coefficient in terms of the external pump rates and the lifetimes. That means

$$
\gamma \rightarrow \gamma_{0}=R_{2} \tau_{2}\left(1-\tau_{10} / \tau_{21}\right) \sigma_{21}
$$

Therefore, (B9) can rewritten in the following manner, 


$$
\gamma\left(I_{21}\right)=\frac{\gamma_{0}}{1+I_{22} / I_{s}}=\frac{k P_{p}}{I+I_{21} / I_{s}}
$$

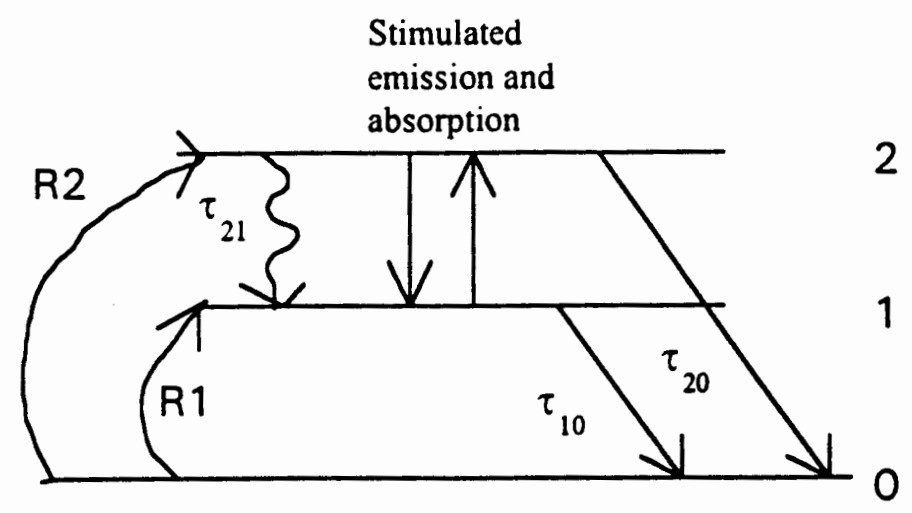

Reservoir of atoms in ground state

Figure B1. Generalized pumping model of a two atomic states laser.

where $\gamma_{0}$ is expressed as the product of a proportionality constant $k$ (units of $1 / \mathrm{W}-\mathrm{cm}$ ) and the pump power $P_{p}$ (units, Watts).

Because, at threshold, the ratio of gain to loss equals unity regardless of whether the laser operates under low (Case 1) or high (Case 2) loss conditions, one can write

$$
\begin{aligned}
& \left.\frac{\gamma_{0}}{\alpha_{\mathrm{T}}}\right|_{\text {case } 1}=\frac{k P_{\mathrm{p}} 1}{\alpha_{\mathrm{T} 1}}=1 \\
& \left.\frac{\gamma_{0}}{\alpha_{\mathrm{T}}}\right|_{\text {case } 2}=\frac{k P_{\mathrm{p}} 2}{\alpha_{\mathrm{T} 2}}=1 .
\end{aligned}
$$

By equating (B12A) to (B12B), and with some simplification, it turns out that the losses for each case are proportional to the respective pump powers, i.e.:

$$
\frac{\alpha_{T 2}}{\alpha_{\mathrm{T} 1}}=\frac{k P_{\mathrm{P}} 2}{k P_{\mathrm{p}} 1}=\frac{\mathrm{P}_{\mathrm{P}} 2}{\mathrm{P}_{\mathrm{P}} 1}
$$

Keep in mind that equation (B13) is valid only at threshold. While it is difficult in practice to accurately measure the threshold pump power for most lasers, it is possible to 
get a good estimate of the threshold pump power by way of a linear regression fit using data for $P_{\text {out }}$ versus $P_{p}$ at higher pump power levels.

To relate (B13) to experimental values, replace $\gamma_{0}$ in (B1) with $\gamma_{0}=k P_{p}$ and then rewrite (B1) in the form of an equation of a straight line.

$$
\begin{aligned}
\text { or } & \mathrm{I}_{0}=\frac{\mathrm{I}_{\mathrm{s}} \cdot \mathrm{kP}_{\mathrm{p}} \cdot \mathrm{T}}{\alpha_{\mathrm{T}}}-\mathrm{I}_{\mathrm{T}} \mathrm{T} \\
\mathrm{y} & =\mathrm{mP}_{\mathrm{p}}-\mathrm{b}
\end{aligned}
$$

where $m=I_{s} k T / \alpha_{T}\left(\mathrm{~cm}^{-2}\right)$ and $b=I_{s} T$, and $y=I_{0}$, the output intensity. By setting $y=0$ (corresponding to zero output from the laser), one can solve (B14) for $P_{p}$ to get

$$
P_{p}=\frac{b}{m}=\frac{I_{s} T}{I_{s} T / \alpha_{T}}=\frac{\alpha T}{k}
$$

which is the same as (B12). By substituting (b/m) for $P_{p}$ in (B12), one gets an expression for the ratio of $\alpha_{\mathrm{T} 2} / \alpha_{\mathrm{T} 1}$ in terms of b's and m's:

$$
\frac{\alpha_{\mathrm{T} 2}}{\alpha_{\mathrm{T} 1}}=\frac{\mathrm{b}_{2} / \mathrm{m}_{2}}{\mathrm{~b}_{1} / \mathrm{m}_{1}}=\frac{\mathrm{b}_{2}}{\mathrm{~b}_{1}} \frac{\mathrm{m}_{1}}{\mathrm{~m}_{2}} \text {. }
$$

The values of b's and m's can be determined by performing a linear regression fit through the data points in Table B2 for both cases. The data in Table B2 along with the regression is shown in Figure B2. Knowing the values for the b's and m's, one can easily determine the ratio of $\alpha_{\mathrm{T} 2} / \alpha_{\mathrm{T} 1}$, and subsequently $\alpha_{\mathrm{T} 2}$ (since the value of $\alpha_{\mathrm{T} 1}$ was determined in previous section). Substituting in the numerical values for b's and m's, the ratio of $\alpha_{\mathrm{T} 2} / \alpha_{\mathrm{T} 1}$ and the value of $\alpha_{\mathrm{T} 2}$ are,

$$
\begin{aligned}
& \frac{\alpha_{\mathrm{T} 2}}{\alpha_{\mathrm{T} 1}}=1.01099 \\
& \alpha_{\mathrm{T} 2}=0.001367\left(\mathrm{~cm}^{-1}\right) .
\end{aligned}
$$

Recall that we set out to determine the ratio of the cavity losses when the AOM is in the high loss and low loss states. The value of $\alpha_{\text {int }}$ for the low loss case was determined 
in section A. By equating equation (B18) to (B2), one can solve for $\alpha_{\text {int }}$ for the hi loss case. The value for $\alpha_{\text {int }}$ high loss is $0.00047 \mathrm{~cm}^{-1}$.

TABLE B2

CONTRIBUTION OF LOSSES DUE TO THE AOM

\begin{tabular}{llll}
\multicolumn{2}{c}{ Case 1 } & \multicolumn{2}{c}{ Case 2 } \\
\hline $\mathbf{P}_{\text {in }}$ & $\mathbf{P}_{\text {out }}$ & $\mathbf{P}_{\text {in }}$ & $\mathbf{P}_{\text {out }}$ \\
952.9 & 5.75 & 955.5 & 4.9 \\
969.2 & 8.35 & 972.0 & 7.6 \\
986.6 & 11.12 & 990.9 & 10.0 \\
1003.3 & 13.5 & 1009.5 & 13.0 \\
1020.0 & 16.75 & 1026.2 & 16.0 \\
1036.9 & 19.25 & 1043.2 & 18.75 \\
1053.9 & 22.0 & 1060.2 & 21.75 \\
1070.8 & 25.0 & 1079.4 & 24.50 \\
\hline
\end{tabular}

The same equation used the previous section to determine round trip loss can be used to determine the loss due to one round trip through the AOM,

$$
\begin{aligned}
& \operatorname{AOM}_{10}=1-\mathrm{e}^{-2 \alpha \sin \mid 0.1} \\
& \mathrm{AOM}_{h i}=1-\mathrm{e}^{-2 \alpha \ln h \cdot 1 \cdot 1} \text {. }
\end{aligned}
$$

From (B19A) one can easily find out the value of the loss due to the AOM in the low loss state. For reasons which will become apparent shortly, rewrite (B19A) as

$$
\begin{aligned}
& \ln \left(1-\mathrm{AOM}_{\mathrm{lo}-\text { loss }}\right)=-2 \alpha_{\text {int lo }} \cdot 1 \\
& \ln \left(1-\mathrm{AOM}_{\mathrm{hi}-\text { loss }}\right)=-2 \alpha_{\text {int hi }} \cdot 1 .
\end{aligned}
$$

But from (B17) we know $\alpha_{\text {int hi }}=1.01099 * \alpha_{\text {int lo }}$, therefore,

$$
\ln \left(1-\mathrm{AOM}_{\mathrm{hi}}\right)=-1.01099 \cdot 2 \alpha_{\mathrm{int} 10} \cdot 1 \text {. }
$$

By substituting (B20) into (B21) for $\left(-2 \alpha_{\text {int lo }} * 1\right)$, raising " $e$ " to this power on both sides, and rearranging a little, one gets 
or

$$
\begin{aligned}
& \mathrm{AOM}_{\mathrm{hi}-\mathrm{loss}}=1-\mathrm{e}^{1.01099 \cdot \ln (1-\mathrm{AOMlo})} \\
& \mathrm{AOM}_{\mathrm{hu}-\text { loss }}=1-\left(1-\mathrm{AOM}_{\mathrm{lo}}\right)^{1.01099} .
\end{aligned}
$$

It is the numerical values from (B19A) and (B22) that are used as high loss and low loss inputs, respectively, in the basic program.

\section{Amount of flashlamp pump power within the $808 \mathrm{~nm}$ region}

After some algebraic manipulation, the amount of pump energy that the rod extracts at the wavelength of $\lambda=0.808 \mathrm{~nm}$ can be estimated. This wavelength corresponds to the output wavelength of a Ga-Al-As diode laser, and can be used to pump the $\mathrm{Nd}$ :YAG system from the ground level to the quartet $F_{5 / 2}\left({ }^{4} F_{5 / 2}\right)$ third excited energy level.

We begin by defining $R_{2}$ in (B 10) with

$$
\mathrm{R}_{2}=\frac{\mathrm{P}_{\mathrm{N} 3}}{\mathrm{~h} \mathrm{~V}_{808} \cdot \mathrm{V}_{\mathrm{m}}}
$$

where $\mathrm{P}_{\mathrm{N} 3}$ is the amount of pump power at $808 \mathrm{~nm}$ that is needed to excite atoms from the ground state to level 3 (Fig. 1). By equating $\gamma_{0}=\mathrm{kP}_{\mathrm{p}}$ with (B10), and rearranging terms, one can obtain an expression for $P_{N_{3}}$ which is proportion to the $P_{p}$.

$$
\begin{gathered}
k P_{p}=\gamma_{0}=\frac{P_{N 3}}{h v_{808} \cdot V_{m}} \cdot \tau_{2} \sigma_{21}\left(1-\tau_{1} / \tau_{21}\right) \\
P_{N_{3}}=\frac{k \cdot h v \cdot V_{m}}{\tau_{2} \sigma_{21}\left(1-\tau_{1} / \tau_{21}\right)} \cdot P_{p}=k_{3} P_{p} .
\end{gathered}
$$

Since we know the value of the slope of the lines in Figure B2, we can calculate the value of $\mathrm{k}$ (units, $1 / \mathrm{W}-\mathrm{cm}$ ). By substituting in the numerical values for all other constants in (B24), $k_{3}$ is found to be $k_{3}=6.9446 * 10^{-4}$. By multiplying the power supplied to the flashiamp (i.e. $P_{\text {in }}=I V$ ) by $k_{3}$, one can estimate the amount of power the lamps emit in the $808 \mathrm{~nm}$ region. 
CVIGAIN.MCD $9 / 3 / 93$

$a^{\prime} s=$ pout in $\mathrm{mW}$ using mirror with radius of curvature ROcl $b^{\prime} s=$ Pout in $\mathrm{mW}$ using mirror with radius of curvature $\mathrm{ROC} 2$
$a_{1}:=79.5$
$a_{2}:=87.5$
$a_{3}:=100$
$a_{4}:=112.5$
$a_{5}:=127.5$ 5
$a_{6}:=145$
$a_{7}:=162.5$

$b_{1}:=6 \quad b_{2}:=10.75 \quad b_{3}:=37 \quad b_{4}:=67.5 \quad b_{5}:=99.5$

$b_{6}:=132.5 \quad b_{7}:=172.5$

nYAG $:=1.82$

Isat $:=1249.65$

$1:=6$

modearea $:=.00424$

ROC1 : $=.9893$

ROC2 $:=.9543$ index of YAG

Saturation intensity (W/ $\left.\mathrm{cm}^{-2} 2\right)$ physical length of the YAG rod (Cm) mode area of the beam at the $O C\left(\mathrm{Cm}^{-2}\right)$ Refelectivity of the output coupler Rocl Refelectivity of the output coupler Roc2

s $:=1 \ldots 7$

$\mathrm{AI}_{\mathrm{s}}:=1+\frac{\mathrm{a} \cdot 10^{-3}}{\text { modearea }} \cdot\left[\frac{1}{\text { Isat }}\right] \cdot\left[\frac{2}{1-\mathrm{ROCl} 1}\right]$

$A 2_{s}:=1+\frac{b \cdot 10^{-3}}{\text { modearea }} \cdot\left[\frac{1}{\text { Isat }}\right] \cdot\left[\frac{2}{1-\text { Roc2 }}\right]$

aint is the cavity loss due to the gain medium only $(1 / \mathrm{cm})$. $\Gamma$ is the small signal gain $(1 / \mathrm{cm})$.

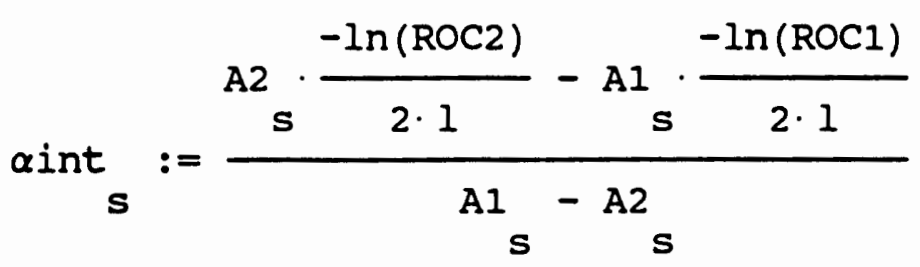


$\Gamma_{s}:=A_{s} \cdot \frac{-\ln (R O C 1)}{2 \cdot 1}+A s_{s} \cdot \alpha i n t s$

below are the value of the gain and the loss for the pump power corresponding to each of the above output values.

A1

\begin{tabular}{|l|}
\multicolumn{1}{c}{$S$} \\
\hline 3.80452358 \\
\hline 4.08673979 \\
\hline 4.52770262 \\
\hline 4.96866545 \\
\hline 5.49782084 \\
\hline 6.1151688 \\
\hline 6.73251676 \\
\hline
\end{tabular}

A2

S

\begin{tabular}{|l|}
\hline 1.04955766 \\
\hline 1.08879081 \\
\hline 1.30560557 \\
\hline 1.55752368 \\
\hline 1.8218312 \\
\hline 2.09439834 \\
\hline 2.42478274 \\
\hline
\end{tabular}

$\alpha$ int

s

\begin{tabular}{|l|}
\hline 0.00024706 \\
\hline 0.00019366 \\
\hline 0.0003198 \\
\hline 0.00047407 \\
\hline 0.00059114 \\
\hline 0.00066706 \\
\hline 0.00079312 \\
\hline
\end{tabular}

$\Gamma$

\begin{tabular}{|l|}
\hline 0.00435058 \\
\hline 0.00445507 \\
\hline 0.00550691 \\
\hline 0.00680976 \\
\hline 0.00817864 \\
\hline 0.00956126 \\
\hline 0.01137518 \\
\hline
\end{tabular}

$$
\alpha_{2}+\operatorname{\alpha int}_{3}+\operatorname{\alpha int}_{4}+\operatorname{\alpha int}_{5}+\operatorname{\alpha int}_{6}
$$

aintavg :=

5

AOM is renaming of the losses excluding the mirror losses ( $\%)$. AOMHi is the additional loss generated when when the AOM acts as an active device ( $\%$ ).

atot is the total loss in the cavity $(1 / \mathrm{cm})$.

RTLoss is the cavity loss in percent.

$\mathrm{AOM}:=1-\left[\exp (\alpha \operatorname{intavg} \cdot 2 \cdot 1)^{-1}\right]$

AOMHi $:=1-(1-\text { AOM })^{1.01099}$

atót := aintavg $+\frac{1}{2 \cdot 1} \cdot \ln \left[\frac{1}{\mathrm{ROCl}}\right]$

RTLoss $:=2 \cdot 1 \cdot \alpha$ tot

ravg $:=\frac{\Gamma_{2}+\Gamma_{3}+\Gamma_{4}+\Gamma_{5}+\Gamma_{6}}{5}$ 
Numerical Values

$$
\begin{array}{ll}
\alpha \text { intavg }=0.00044915 & 1 / \mathrm{cm} \\
\alpha \text { tot }=0.00134562 & 1 / \mathrm{cm} \\
\Gamma \text { avg }=0.00690233 & 1 / \mathrm{cm} \\
\text { AOM }=0.00537525 & (\%) \\
\text { AOMHi }=0.00543416 & (\%) \\
\text { RTLOSs }=0.01614741 & (\%)
\end{array}
$$




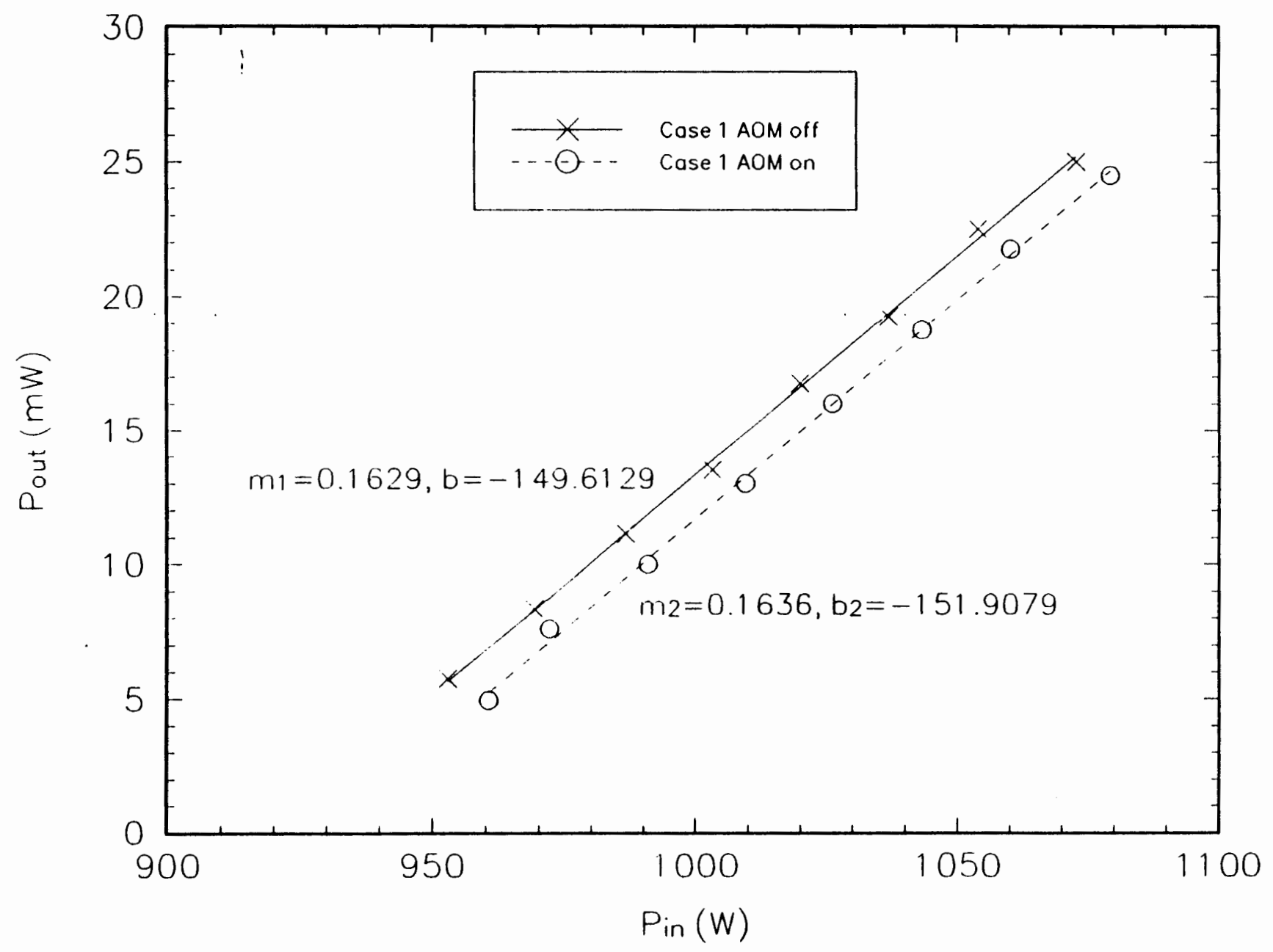

Eigure B2. $P_{\text {out }}$ versus $P_{\text {in }}$ for the AOM in the low loss (case 1) and high loss (case 2) states. 


\section{APPENDIX C}

BOLTZMANN DISTRIBUTION OF ATOMS IN Nd:YAG 
According to statistical thermodynamics, when a large collection of similar atoms is in thermal equilibrium at temperature $T$, the relative populations of any two energy levels $E_{1}$ and $E_{2}$ must be related by the Boltzmann ratio

$$
\frac{N_{2}}{N_{1}}=\exp \left[-\frac{\left(E_{2}-E_{1}\right)}{k T}\right]
$$

where $N_{2}$ and $N_{1}$ correspond to the population of atoms in energy levels $E_{2}$ and $E_{1}$, respectively, [5], and $k$ is the Boltzmann constant. For energy gaps large enough such that $E_{2}-E_{1}=h v>>k T$, the ratio is approximately zero, and there will be few atoms in the upper energy level at thermal equilibrium.

Using equation ( $\mathrm{Cl}$ ), the Boltzmann's population of energy levels ${ }^{4} I_{11 / 2},{ }^{4} F_{3 / 2}$, and ${ }^{4} F_{5 / 2}$ (which correspond to levels $1,2,3$ respectively in the simplified energy level diagram in Figure 1 in Chapter I) are calculated below. The population of the ground state ${ }^{4} I_{9 / 2}$ is determined by the product of the mode volume $\mathrm{V}_{\mathrm{m}}$ and the doping of $\mathrm{Nd}^{+3}$ ion.

I). Population of Level 1.

$$
\begin{aligned}
& \frac{E_{1}}{k T}=\frac{h v_{1}}{k T}=\frac{h c}{\lambda_{1} k T}=\frac{\left(6.626 \cdot 10^{-34}\right)\left(3 \cdot 10^{8}\right)}{\left(4.739 \cdot 10^{-6}\right)\left(4.14 \cdot 10^{-21}\right)}=10.132 \\
& N_{1}=N_{0} \mathrm{e}^{-E 1 / k T}=3.513 \cdot 10^{18} \mathrm{e}^{-10.132} \\
& N_{1}=1.39767 \cdot 10^{14}
\end{aligned}
$$

II). Population of Level 2.

$$
\frac{\mathrm{E}_{2}}{\mathrm{kT}}=\frac{\mathrm{h} v_{2}}{\mathrm{kT}}=\frac{\mathrm{hc}}{\lambda_{2} \mathrm{kT}}=\frac{4.801 \cdot 10^{-5}}{0.869 \cdot 10^{-6}}=55.25
$$

$$
\begin{aligned}
& \mathrm{N}_{2}=\mathrm{No}_{0} \mathrm{e}^{-\mathrm{E} 2 / \mathrm{kT}}=3.513 \cdot 10^{18} \mathrm{e}^{-55.25} \\
& \mathrm{~N}_{2}=3.556 \cdot 10^{-6} \approx 0
\end{aligned}
$$


III). Population of Level 3.

$$
\begin{aligned}
& \frac{E_{3}}{k T}=\frac{h v_{3}}{k T}=\frac{h c}{\lambda_{3} k T}=\frac{4.801 \cdot 10^{-5}}{0.808 \cdot 10^{-6}}=59.395 \\
& N_{3}=N_{0} \mathrm{e}^{-E 3 / k T}=3.513 \cdot 10^{18} \mathrm{e}^{-59.39} \\
& N_{3}=5.661 \cdot 10^{-8} \approx 0
\end{aligned}
$$

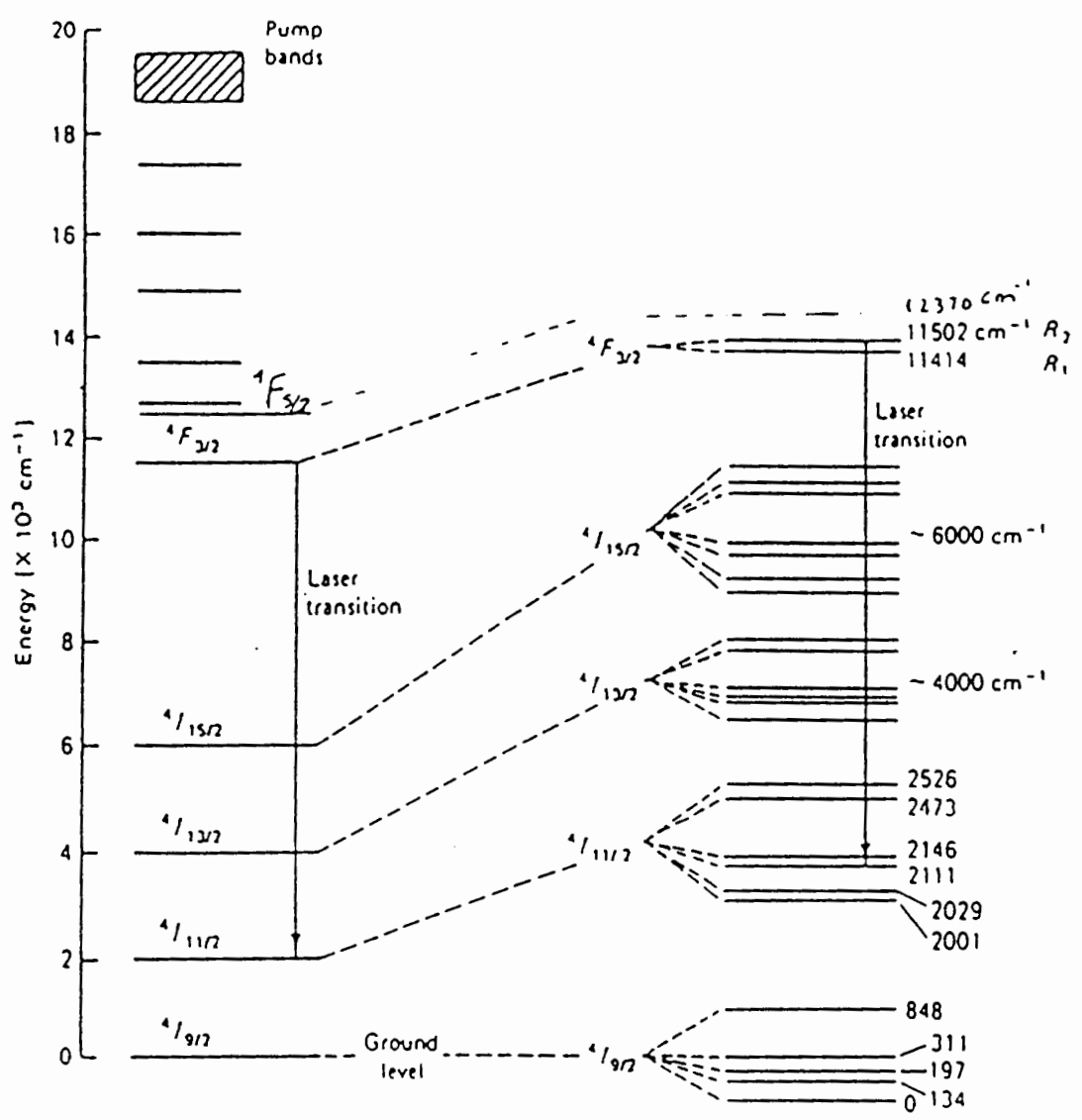

Figure C1. Energy level diagram of Nd:YAG [5]. 
APPENDIX D

LINEARIZED RATE EQUATIONS 
Small signal analysis by definition implies that the value of the perturbations of all variables involved are small with respect to some steady state value. In this section the perturbations will be introduced as loss modulation. Assume solutions to equations (2) through (5) in chapter 1 are:

$$
\begin{aligned}
& N_{1}=\bar{N}_{1}+\Delta N_{1} e^{-j \omega t} \\
& N_{2}=\bar{N}_{2}+\Delta N_{2} e^{-j \omega t} \\
& N_{3}=\bar{N}_{3}+\Delta N_{3} e^{-j \omega t} \\
& I_{21}=I_{D c}+\Delta \mathrm{Ie}^{-j \omega t}
\end{aligned}
$$

where the first term in each solution represents the steady state value for that variable, and the second term is a small perturbation around the steady state value. To allow for any phase differences between the perturbation and the original signal, the second term also includes an $e^{-j \omega t}$ term where $\omega$ represents the modulation frequency. For gain modulation, assume that the pumping also deviates from a steady state value in the same manner, i.e.:

$$
\mathrm{P}=\overline{\mathrm{P}}+\Delta \mathrm{Pe}^{-\mathrm{j \omega \omega}}
$$

To get an expression for the change in intensity $\Delta \mathrm{I}$ as a function of pump fluctuation, we begin by substituting (D1) through (D5) back into their respective rate equation given in chapter 1 , equate the $e^{-j \omega t}$ terms, and solve for the $\Delta \mathrm{N}$ 's and $\Delta \mathrm{I}$. The steady state needed in the above procedure can either be obtained from the results of the Quick Basic program (CWJHONID.EXE) or calculated in the Mathcad program (DELTAI.MCD) just prior to calculating the transient values. Using the above outlined procedure one arrives at the following expressions for the $\Delta \mathrm{N}$ 's and $\Delta \mathrm{I}$ :

$$
\begin{gathered}
\Delta N_{3}=\frac{\Delta P \tau_{3}}{1-j \omega \tau_{3}} \\
\Delta N_{2}=\frac{\sigma_{21} I_{D c} \Delta N_{1}-\sigma_{21} \Delta I \Delta N_{c}+\Delta N_{3} / \tau_{32}}{\sigma_{21} I_{b c}+1 / \tau_{2}-j \omega}
\end{gathered}
$$




$$
\begin{gathered}
\Delta N_{1}=\frac{\sigma_{21} I_{D c} \Delta N_{2}+\sigma_{21} \Delta I \Delta N_{c}+\Delta N_{2} / \tau_{21}}{\sigma_{21} I_{D c}+1 / \tau_{10}-j \omega} \\
\Delta I=\frac{j\left(\Delta N_{2}-\Delta N_{1}\right) L_{b c}}{\tau_{c a v} \cdot \omega \cdot \Delta N_{c}}
\end{gathered}
$$

where $\left(\overline{\mathrm{N}}_{2}-\overline{\mathrm{N}}_{1}\right)=\Delta \mathrm{N}_{\mathrm{c}}$.

In arriving at the final expression for $\Delta \mathrm{I}$, the two terms involving $1 / \tau_{\mathrm{cav}}$ in the denominator summed to zero. The justification for doing so is that $\Delta \mathrm{I}$ is strongly dependent on cavity losses introduced by the AOM since $\tau_{\text {low ioss }}>\tau_{\text {cav }}$. If one tries to solve for $\Delta \mathrm{I}$ by substituting (D6) through (D8) in (D9), the resulting expression appears to be quite complicated and seemingly unsolvable. However, if (D6) through (D9) were written in matrix form, then the value for $\Delta \mathrm{I}$ can be obtained quite easily using Cramer's Rule. Rewriting (D6) through (D9) in matrix form one gets,

$$
\begin{aligned}
{\left[\begin{array}{ccc}
-\mathrm{K}_{1} & 1 & \mathrm{~K}_{2} \\
-1 & \mathrm{~K}_{4} & \mathrm{~K}_{5} \\
\mathrm{~K}_{6} & -\mathrm{K}_{6} & 1
\end{array}\right]\left[\begin{array}{c}
\Delta \mathrm{N}_{1} \\
\Delta \mathrm{N}_{2} \\
\Delta \mathrm{I}
\end{array}\right] } & =\left[\begin{array}{c}
\mathrm{K}_{3} \\
0 \\
0
\end{array}\right] \\
{\left[\mathbf{M}^{2}\right] \quad[\mathbf{X}] } & =[\mathbf{C}]
\end{aligned}
$$

where

$$
\begin{gathered}
K_{1}=\frac{\sigma_{21} I_{D C}}{\sigma_{21} I_{D C}+1 / \tau_{2}-j \omega}=\frac{\sigma_{21} I_{D C}}{D_{2}} \\
K_{2}=\frac{\sigma_{21} \Delta N_{C}}{D_{2}} \\
K_{3}=\frac{\Delta N_{3}}{\tau_{32}} \cdot \frac{1}{D_{2}}=\Delta P \frac{\tau_{3}}{\tau_{32}} \cdot \frac{1}{D_{2}} \\
K_{+}=\frac{\sigma_{21} I_{D C}+1 / \tau_{21}}{\sigma_{21} I_{D C}+1 / \tau_{10}-j \omega}=\frac{\sigma_{21} I_{D C}+1 / \tau_{21}}{D_{1}}
\end{gathered}
$$




$$
\begin{aligned}
& \mathrm{K}_{\mathrm{s}}=\frac{\sigma_{21} \Delta \mathrm{N}_{\mathrm{c}}}{\mathrm{D}_{1}} \\
& \mathrm{~K}_{6}=\mathrm{j} \frac{\mathrm{I} \mathrm{Dc}}{\Delta \mathrm{N}_{\mathrm{c}} \tau_{\mathrm{cav}} \omega} .
\end{aligned}
$$

Using Cramer's Rule as suggested above, the solution to $\Delta \mathrm{I}$ is

$$
\Delta I=\left|\begin{array}{ccc}
-\mathrm{K} 1 & 1 & \mathrm{~K} 3 \\
-1 & \mathrm{~K} 4 & 0 \\
\mathrm{~K} 6 & -\mathrm{K} 6 & 0
\end{array}\right| \cdot \frac{1}{\operatorname{det} \mathbf{M}}
$$

where

$$
\operatorname{det} M=-K_{1} K_{4}+K_{5} K_{6}\left(1-K_{1}\right)+K_{2} K_{6}\left(1-K_{4}\right)+1
$$

is the determinant of the matrix defined in (D10). Writing (D18) in an equation form one gets

$$
\Delta I=\frac{K_{3} K_{6}\left(1-K_{4}\right)}{1-K_{1} K_{4}+K_{5} K_{6}\left(1-K_{1}\right)+K_{2} K_{6}\left(1-K_{4}\right)}
$$

After some rearranging, the above expression reduces to

$$
\Delta I=\frac{K_{3}}{\frac{1-K_{1} K_{+}}{K_{6}\left(1-K_{+}\right)}+K_{5} \frac{1-K_{1}}{1-K_{4}}+K_{2}} .
$$

Since a numerical value for $\Delta \mathrm{I}$ by itself has little meaning, let us introduce the term modulation depth $\xi$ as being a measure of the output intensity fluctuation $(\Delta I)$, as a function of pump power and modulation frequency, relative to the steady state value. In equation form $\xi$ is defined as:

$$
\xi=|\Delta \mathrm{I}| / \mathrm{I}
$$

To evaluate the above expression for a range of pump powers and modulation frequencies a Mathcad program (DELTAI.MCD) was written. A listing of the program is included at the end of this appendix. 
DELTAI5.MCD 11-5-93

This program calculates the output fluctuations arising from modulation of the pump source (or gain modulation) by calculating the steady state population and intracavity intensity. Subsequently, the expression for output intensity fluctuation derived earlier in this appendix is evaluated is evaluated.

nYAG $:=1.82$

$1:=6$

modearea $:=.00424$

$\sigma 21:=6.5 \cdot 10$

hv1064 $:=1.868 \cdot 10$ $-19$

$-19$

hv808 $:=2.4601 \cdot 10$ 10

$c:=3 \cdot 10$

RTLOSS $:=.016145$

ROCI : $=.9893$

OPL $:=32.77$

$\tau 10:=11 \cdot 10^{-9}$

$-6$

$\tau 20:=395 \cdot 10$

$\tau 21:=550 \cdot 10^{-6}$

$-6$

$\tau 30:=50 \cdot 10$

$-9$

energy of photons at $1064 \mathrm{~nm}$

energy of photons at $808 \mathrm{~nm}$

speed of light (cm/s)

total round trip loss (q)

Reflectivity of output coupler optical path length of cavity

time constants from Table I

$\tau 2:=\left[\frac{1}{\tau 20}+\frac{1}{\tau 21}\right]^{-1}$
$\tau 3:=\left[\frac{1}{\tau 30}+\frac{1}{\tau 32}\right]^{-1}$

$\tau$ cav $:=\left[2 \cdot \frac{\mathrm{OPL}}{\mathrm{c}}\right] \cdot \frac{1}{\text { RTLOSS }}$

photon decay time ( $\mu s)$

modvol $:=1 \cdot$ modearea

mode volume $\left(\mathrm{cm}^{-} 3\right)$ 


$$
\begin{array}{ll}
\delta \mathrm{NC}:=\frac{\operatorname{modvol} \cdot \mathrm{nYAG}}{\tau \mathrm{cav} \cdot \sigma 21 \cdot \mathrm{c}} & \text { steady state pop. inversion } \\
\text { No }:=1.38 \cdot 10^{20} \cdot \text { modvol } & \text { ground state population } \\
\text { N1B }:=\text { No } \exp (-10.132) & \begin{array}{l}
\text { level } 1 \text { population due to } \\
\text { Boltzman distribution }
\end{array}
\end{array}
$$

$$
\begin{aligned}
& \text { CWPUMP }:=2.0 \quad \text { input pump power (W) } \\
& P:=\frac{\text { CWPUMP }}{\text { hV808 }} \quad \begin{array}{l}
\text { convert input power to \# of } \\
\text { photons at } 808 \mathrm{~nm}
\end{array} \\
& \text { N3 := P. } \tau 3 \quad \text { pop. of levels } 3,2,1 \\
& \mathrm{~N} 1:=\left[\mathrm{N} 3 \cdot \frac{\tau 20}{\tau 32}+\mathrm{N} 1 \mathrm{~B} \cdot \frac{\tau 20}{\tau 10}-\delta \mathrm{NC}\right] \cdot \frac{\tau 10}{\tau 10+\tau 20} \\
& \mathrm{~N} 2:=\left[\mathrm{N} 3 \cdot \frac{\tau 20}{\tau 32}-(\mathrm{N} 1-\mathrm{N} 1 \mathrm{~B}) \cdot \frac{\tau 20}{\tau 10}\right]
\end{aligned}
$$

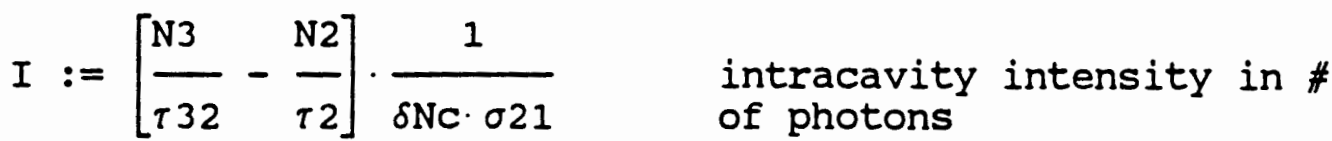$$
\text { Pout }:=\frac{1}{2} \cdot(1-\text { ROC1 }) \cdot \text { modearea } \cdot \text { hv1064 I }
$$

Lowerf $:=50000$

Upperf $:=1 \cdot 10$

Npt $:=400$

s : $:=1 \ldots$ Npt 
$\delta f:=\frac{\log \left[\frac{\text { Upperf }}{\text { Lowerf }}\right]}{\text { Npt }} \quad$ step size

$f_{s}:=10^{\delta f \cdot s+\log (\text { Lowerf })}$

$w_{s}:=f_{s} \cdot 2 \cdot \pi$

$\delta P:=.05 \cdot P$

all the D's and K's are defined on pg 98-99

$D 1_{s}:=\left[\sigma 21 \cdot I+\frac{1}{\tau 10}-i \cdot w_{s}\right] \quad$ D2 $:=\left[\sigma 21 \cdot I+\frac{1}{\tau 2}-i \cdot w{ }_{s}\right]$

$\delta \mathrm{N} 3 \mathrm{~s}:=\frac{\tau 3 \cdot \delta \mathrm{P}}{1-i \cdot \mathrm{w} \cdot \tau 3}$

$\mathrm{K} 1 \mathrm{~s}:=\frac{\sigma 21 \cdot \mathrm{I}}{\mathrm{D} 2}$

$\mathrm{K} 2:=\frac{\sigma 21 \cdot(\mathrm{N} 2-\mathrm{N} 1)}{\mathrm{D} 2}$

$\mathrm{R3}:=\frac{\delta \mathrm{N} 3}{\tau 32 \cdot \mathrm{D} 2}$

$\sigma 21 \cdot I+\frac{1}{\tau 21}$

$\mathrm{K5} s:=\frac{\sigma 21 \cdot(\mathrm{N} 2-\mathrm{N} 1)}{\mathrm{D} 1}$

$K \sigma_{s}:=\frac{i \cdot I}{(\mathrm{~N} 2-\mathrm{N} 1) \cdot \tau \mathrm{Cav} \cdot \mathrm{w}} \mathrm{s}$

$\operatorname{deltaI}_{\mathrm{s}}:=\frac{\mathrm{K} 3 \cdot \mathrm{K} 6 \cdot \mathrm{s}\left[\begin{array}{l}1-\mathrm{K} 4 \\ \mathrm{~s}\end{array}\right]}{1-\mathrm{K} \mathrm{s}_{\mathrm{s}} \cdot \mathrm{K} 4 \mathrm{~s} \mathrm{~S}_{\mathrm{s}} \cdot \mathrm{K} 6 \cdot\left[\begin{array}{r}1-\mathrm{K} 4 \\ \mathrm{~s}\end{array}\right]+\mathrm{K} 5 \cdot \mathrm{K} 6 \cdot\left[\begin{array}{r}1-\mathrm{K} 1 \\ \mathrm{~s}\end{array}\right]}$ 
$\operatorname{Amp\delta I}_{\mathbf{s}}:=\left|\begin{array}{rl}\text { deItaI } \\ \mathrm{s}\end{array}\right|$

Moddepth $:=\frac{\operatorname{Amp} \delta I_{\mathrm{s}}}{I}$

WRITEPRN (FREQ) $:=f$

WRITEPRN (MOD6) $:=$ Moddepth

write the mod. freq. and the mod. depth to PRN files

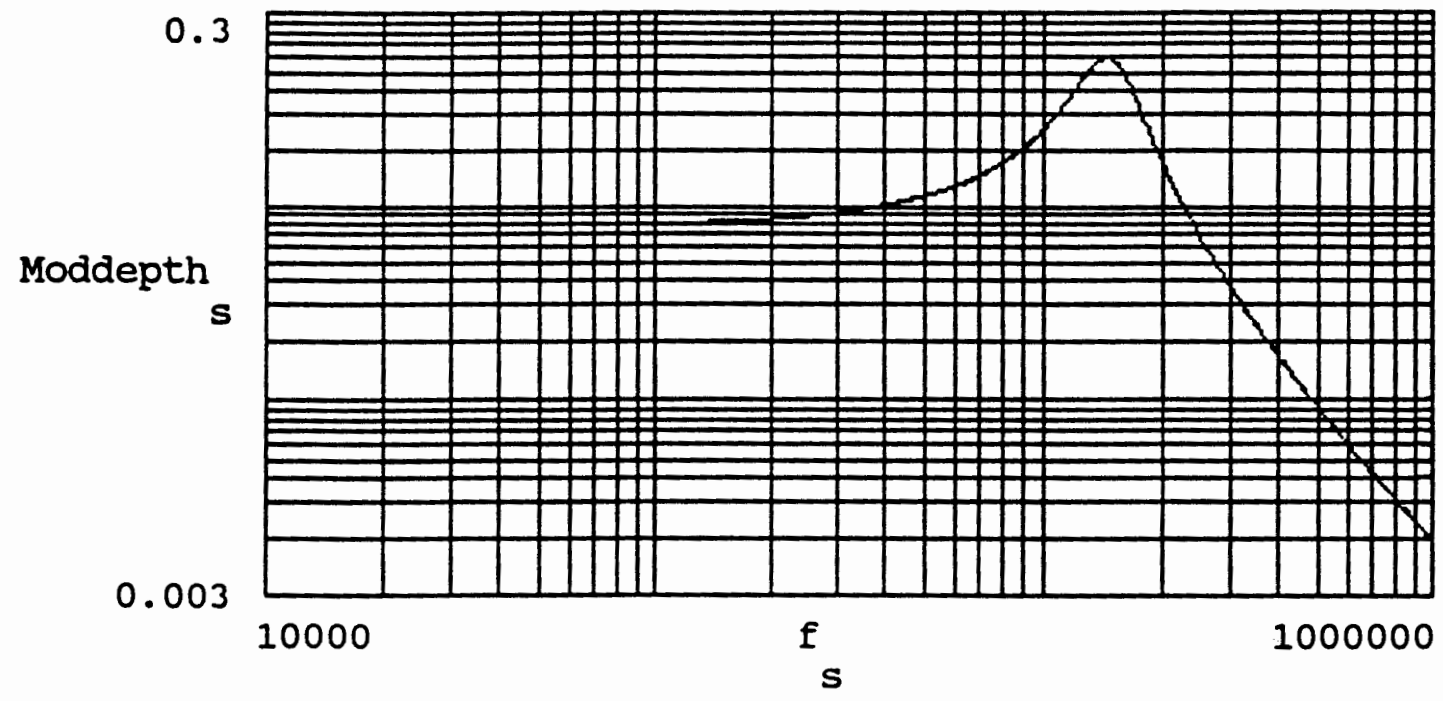




\section{APPENDIX E}

NUMERICAL SOLUTIONS TO THE RATE EQUATIONS 
This appendix includes the listing of a program, written using Microsoft Quick Basic, to generate numerical results of the coupled nonlinear rate equations listed at the beginning of Chapter II. To minimize round off errors resulting from arithmatic operation involving number like $10^{-34}$ for Planck's constant and $10^{19}$ for the $\mathrm{Nd}$ doping oncentration, the units of ' $\mathrm{mm}$ ' and ' $\mu \mathrm{s}$ ' were used wherever possible.

To determine the accuracy of the program, a number of simulations were run using artificially long time constants for $\tau_{20}, \tau_{21}$, and $\tau_{32}$, like 1 second, and checking to see if the ratio of the output-to-input power approached the quantum efficiency of the system. In testing this hypothesis the assumption is made that if there are no non-radiative losses in the laser, the laser should operate at the quantum efficiency of $75.6 \%$ which is obtained by taking the ratio of the pump wavelength to that of the output wavelength. The resulsts of the simulations show that when there are no non-radiative losses, the output of the laser is $75.2 \%$ which is equals the theoretical quantum efficiency. 
This program solves the rate equations using Runga-Kutta method. To 'minimize the round off errors due to multiplication/division of large 'numbers by small numbers (and vice versa), will use mm \& us units

'wherever possible.

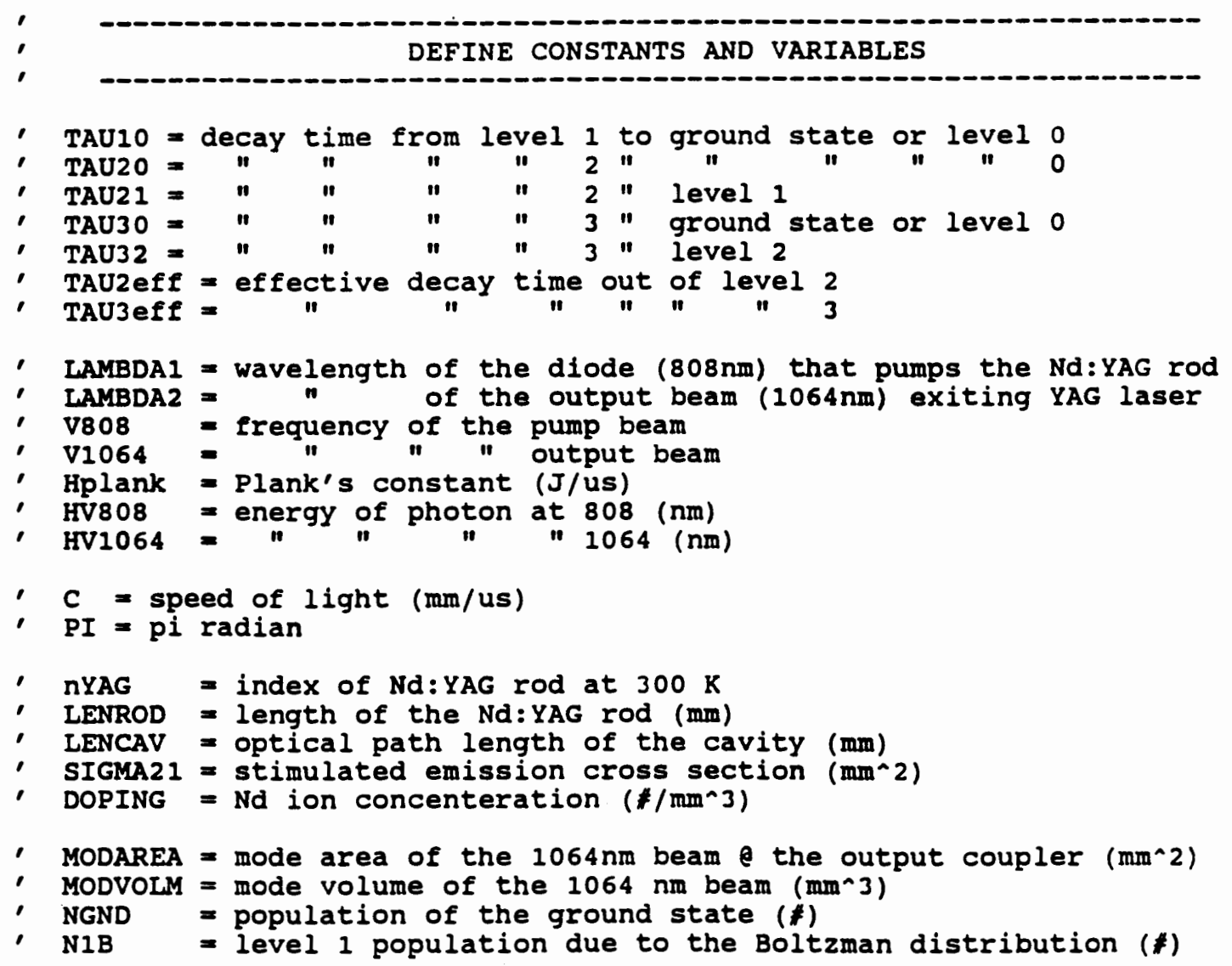

DECLARE SUB RUNga (PUMP)

DIM Ibwd(500), Ifwd(500) AS DOUBLE

DIM SHARED $N(3)$ AS DOUBLE

DIM SHARED YN(3) AS DOUBLE

DIM SHARED YERROR ( 3 ) AS DOUBLE

DIM TSTART (3600), TEND (3600) AS DOUBLE

DIM RSTART ( 300$)$, REND(300) AS DOUBLE

DIM P(500) AS DOUBLE

DIM SHARED TK1 (3), TK2(3), IPRIME(3) AS DOUBLE

COMMON SHARED TAU10, TAU20, TAU21, TAU30, TAU32, TAU2eff AS DOUBLE COMMON SHARED TAU3ef, FSIGMA21, SIGMA03, COEF21, COEFOUT AS DOUBLE 


\begin{abstract}
COMMON SHARED NGND, N1B, DELTAT, DELTAZ, DEL2, DEL6 AS DOUBLE COMMON SHARED CWPUMP, PUMP, SIMTIME AS DOUBLE COMMON SHARED YI, $T$, twindOW, RTT, TOUT, RTLOSS AS DOUBLE COMMON SHARED IOId, IlOSS, IOUT AS DOUBLE COMMON SHARED EPcoef, ELcoef, Epump, ECav, Eout AS DOUBLE COMMON SHARED C, ElOSS, EN1, EN2, EN3, Ebalanc AS DOUBLE COMMON SHARED LAMBDA1, LAMBDA2, V808, V1064, HV808, HV1064 AS DOUBLE
\end{abstract}

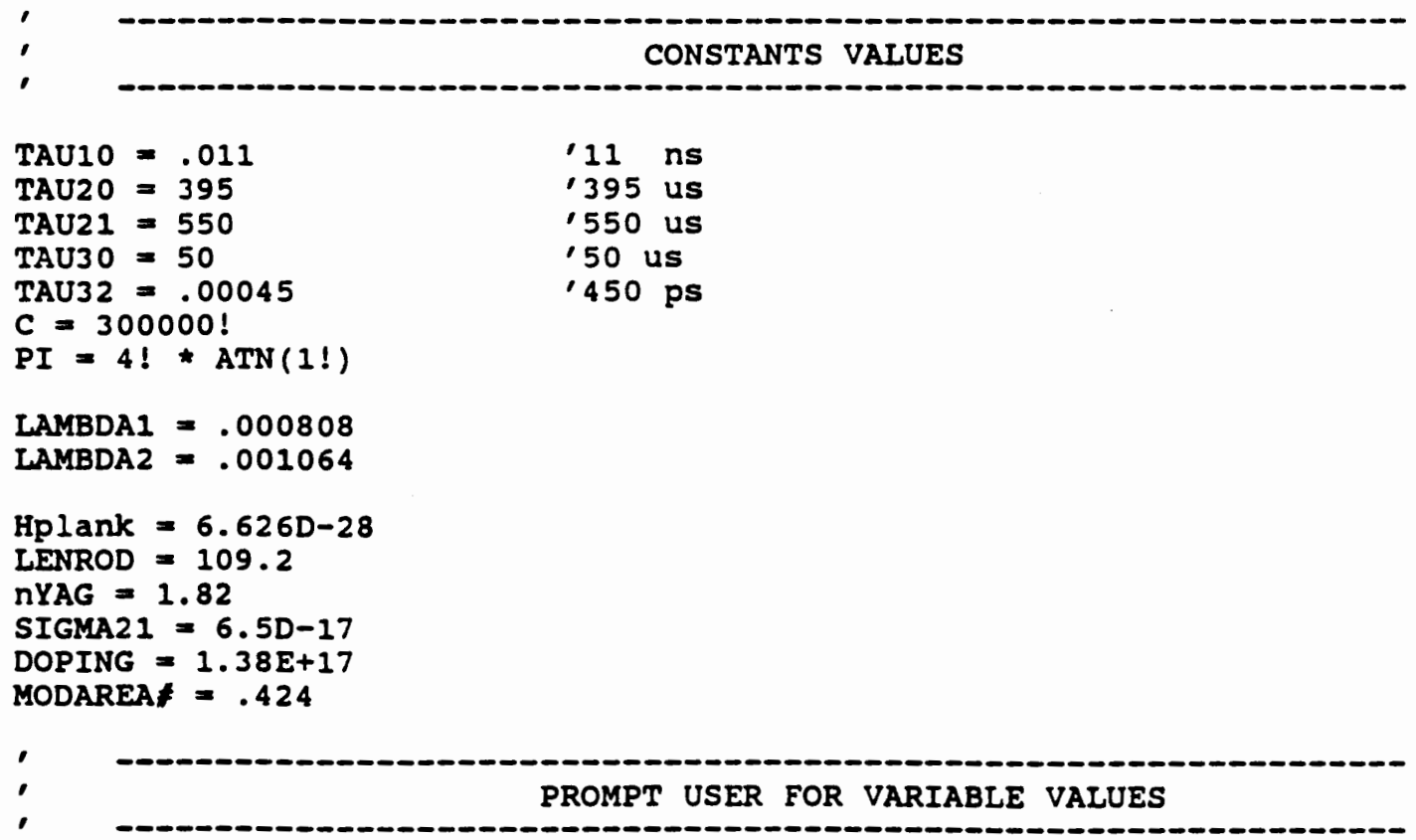

The following loop writes the user input values to a file (filename.inp) 'that can be called up when executing the cwjhonld.exe program in a 'sequential manner.

CLS

INPUT "Enter a .INP filename (w/0.INP) or hit return "; dum\$ IF dums <> " "THEN

OPEN dumS + ". InP" FOR INPUT AS \#4

FOR Vt $=1$ TO 13

INPUT $\$ 4, \mathrm{Xx} \$$

PRINT XXS

NEXT v'

IF EOF (4) THEN

CLOSE $\$ 4$

ELSE

INPUT \#4, $x \times \$$

PRINT XX\$

INPUT \#4, XX\$

PRINT XXS

END IF

CLOSE \#4

END IF

LENCAV $=327.7$ 


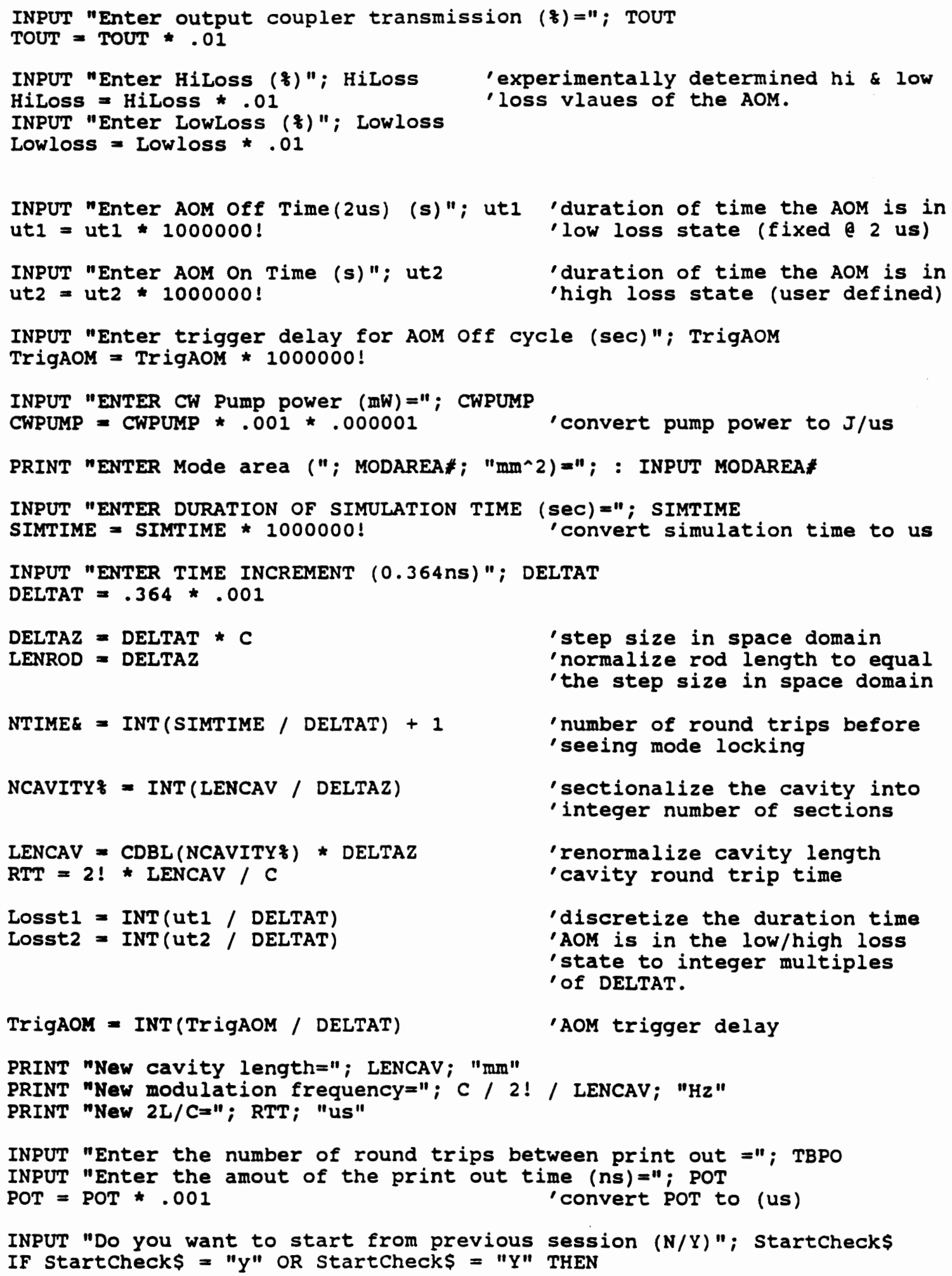


startcheck\$ = "Y"

INPUT "Enter previous status file name (w/0.STA)"; statusfiles statusfiles = statusfiles + ".STA" END IF

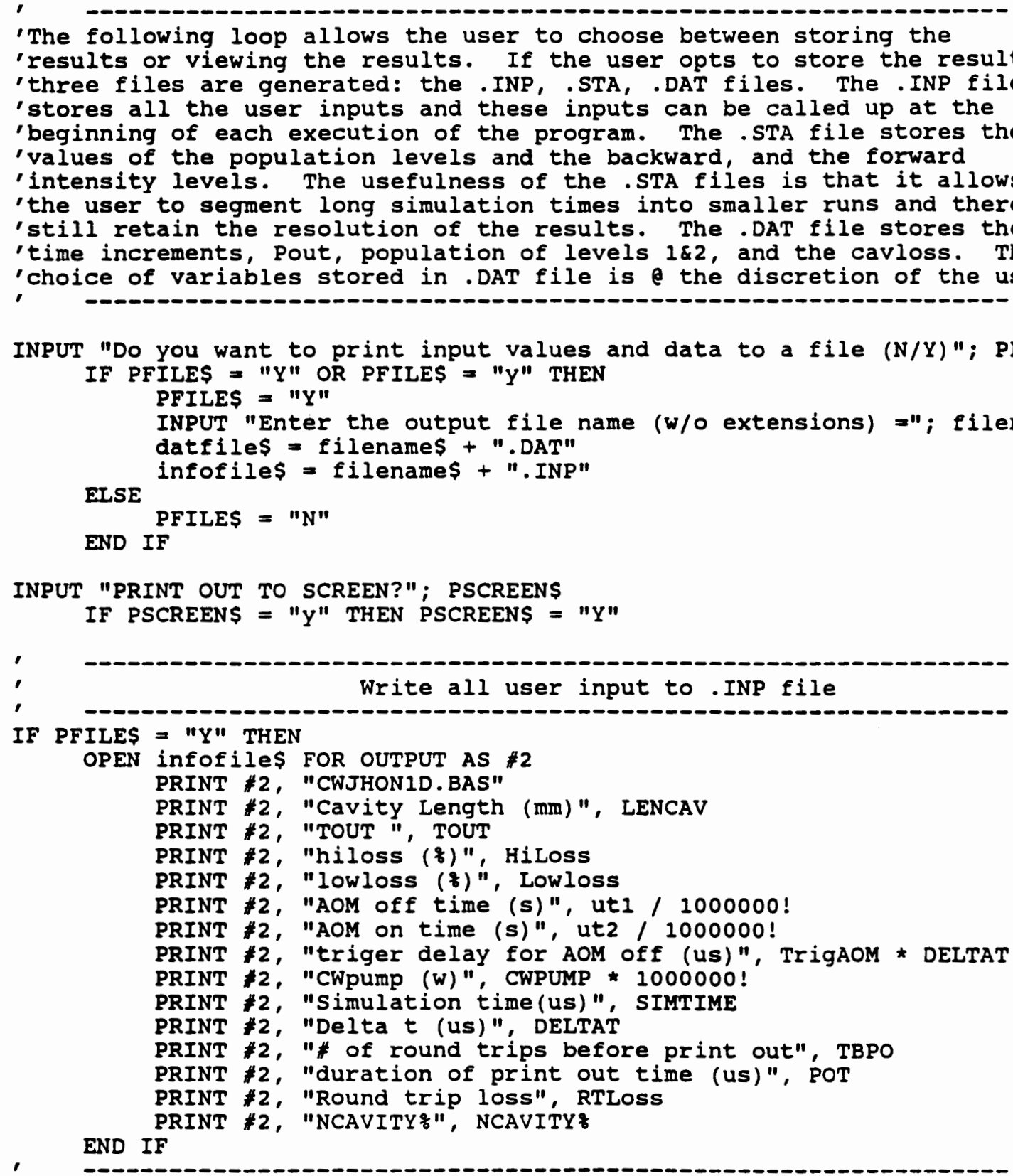




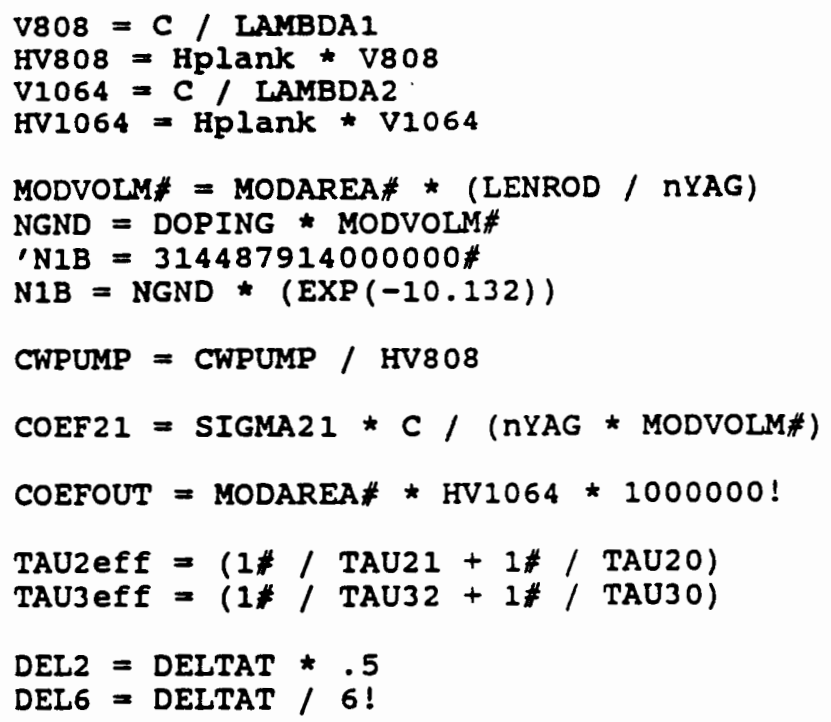

'DEL2 \& DEL6 are used in the 'Runga as dummy variables

'Print out values of some of the variables for reference check before 'starting the simulation.

PRINT "hv808="; HV808; " hV1064="; HV1064

PRINT "mode area="; MODAREA\#; " MOdvolm="; MODVOLM\#; " Lenrod="; LENROD; PRINT " CWPUMP="; CWPUMP; " MOdPOW="; MODPOW;

PRINT " N1B="; N1B; " SIGMA21="; SIGMA21; "COEF21="; COEF21

PRINT "TAU2eff="; TAU2eff; " TAU3eff="; TAU3eff; "COEFOUT="; COEFOUT; INPUT q\$

\section{INITIAL CONDITIONS}

'If the user wants to continue simulating from the termination of a 'previous execution, the values for the population of each level and 'intensities are taken from the .STA file gernerated in the previous 'execution. otherwise, all values except Nl are initialized to 1. 'N1 is set equal to N1B.

IF Startcheck\$ = "Y" THEN

OPEN statusfileS FOR INPUT AS \#3

INPUT \#3, TPREV

PRINT "Simulation time at the end of last sessione="; TPREV INPUT \#3, $N(0), N(1), N(2)$, RTLOSs

FOR $2 I z=1$ TO NCAVITY INPUT \#3, Ifwd ( $2 I \%)$ INPUT $\# 3$, Ibwd ( $2 I \%)$

NEXT 2I\%

INPUT \#3, Ozp\%

INPUT \#3, Epump

INPUT $\# 3$, Eloss

CLOSE \#3

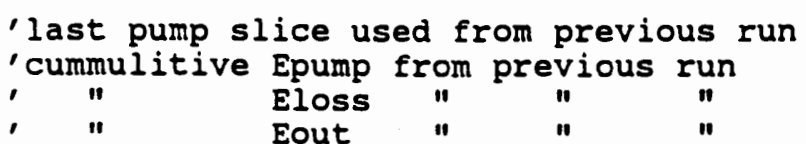
'cummulitive Epump from previous run Eout

ELSE

$N(1)=N 1 B !$

'initial populat'n of level 1 (\# atoms) 


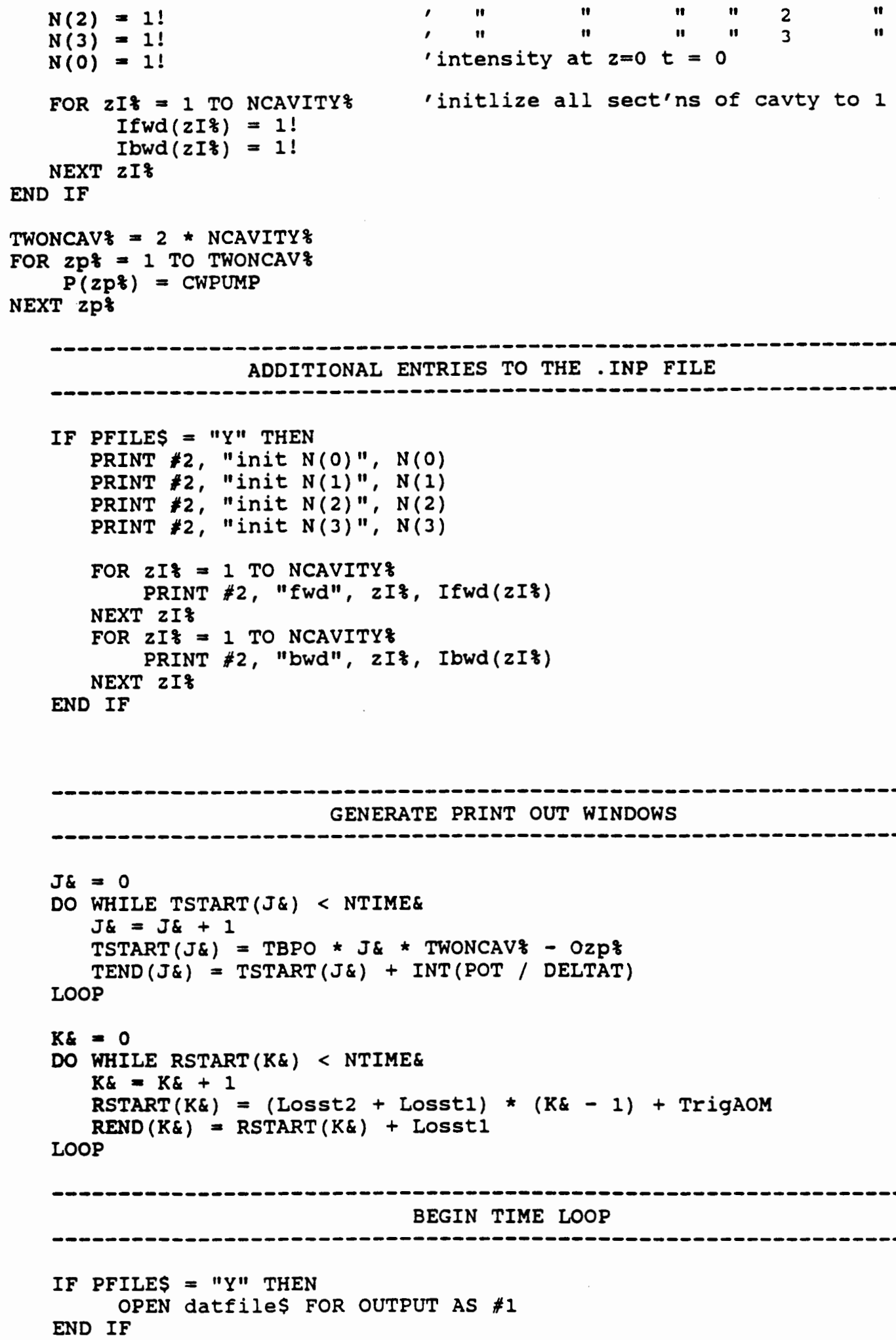




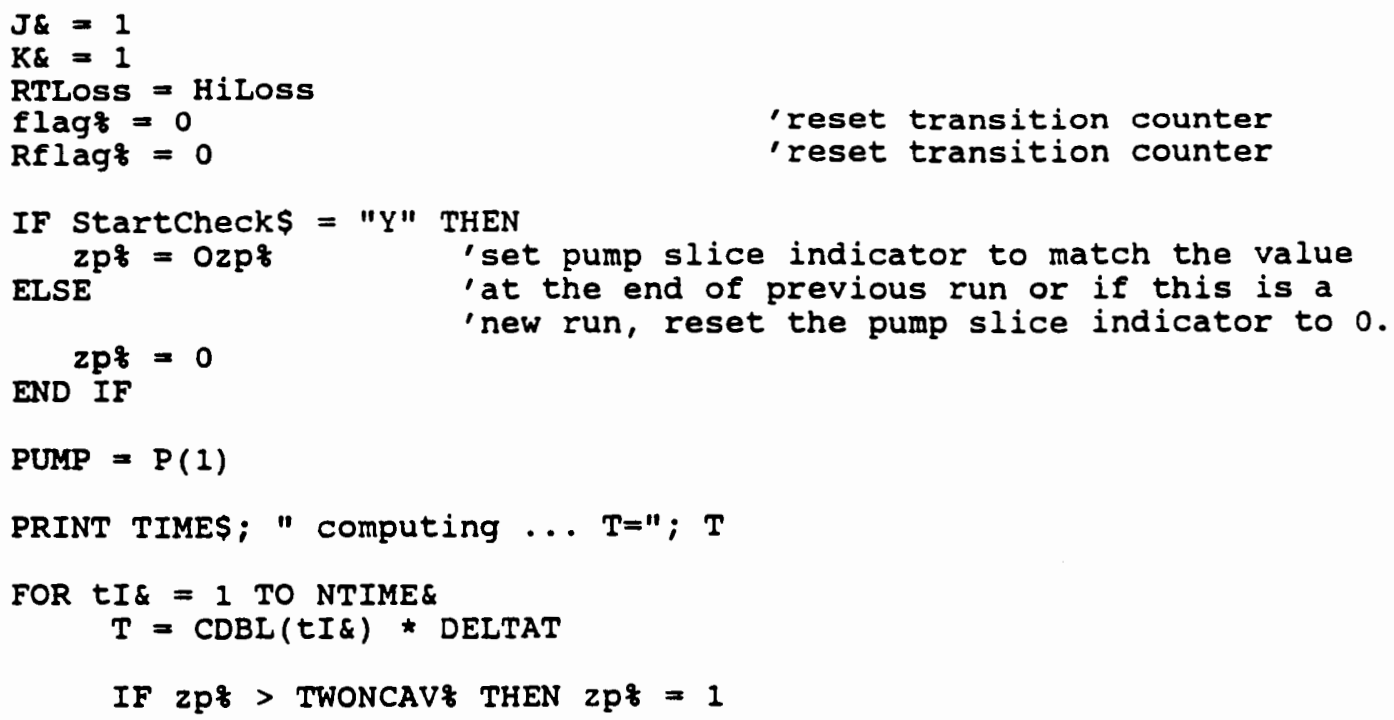

'Pout is multiplied by 1000 to display the value in $\mathrm{mW}$.

'RTLoss is multiplied by 100 to display the value in percent.

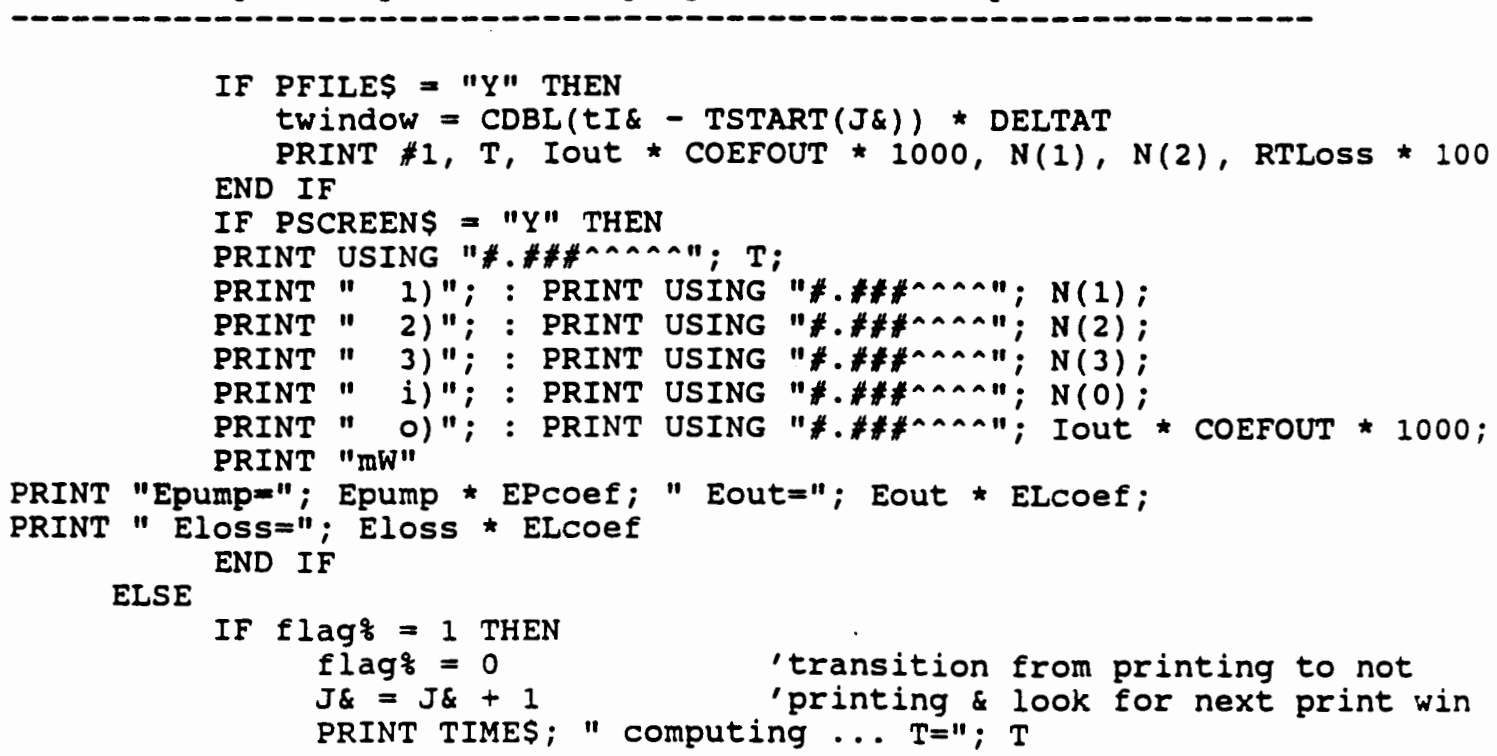




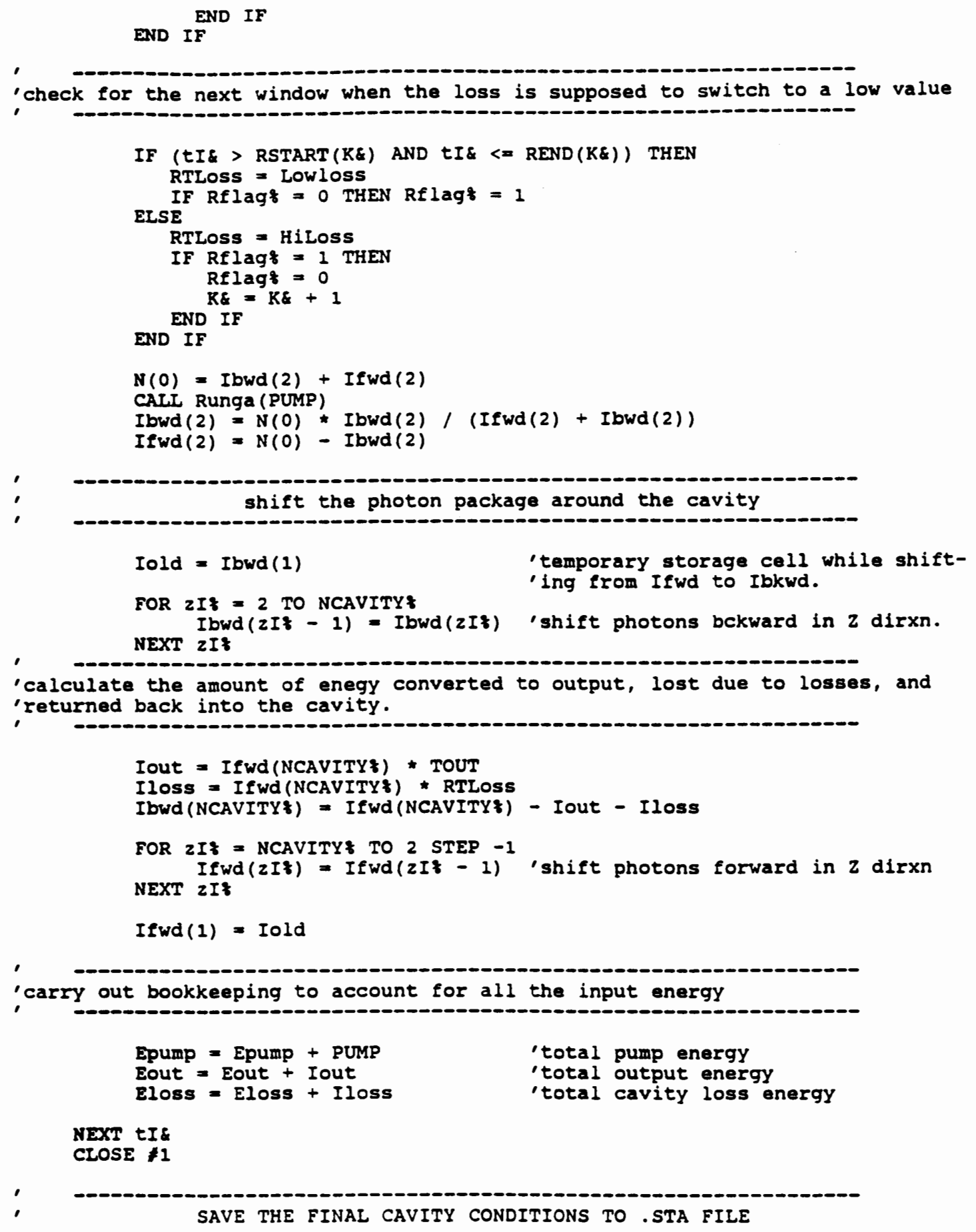




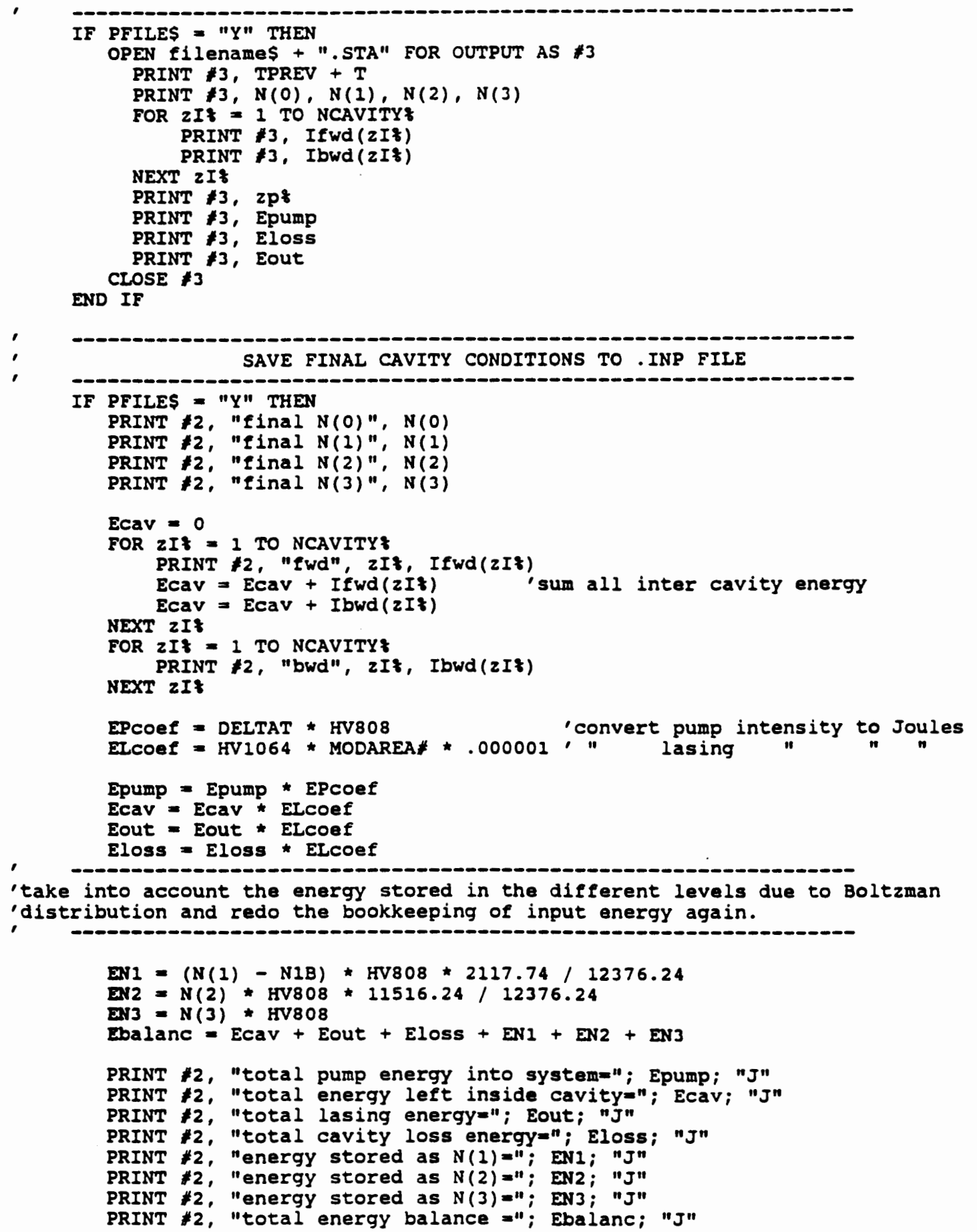


PRINT 2 , "final power out ="; Iout * COEFOUT; "W"

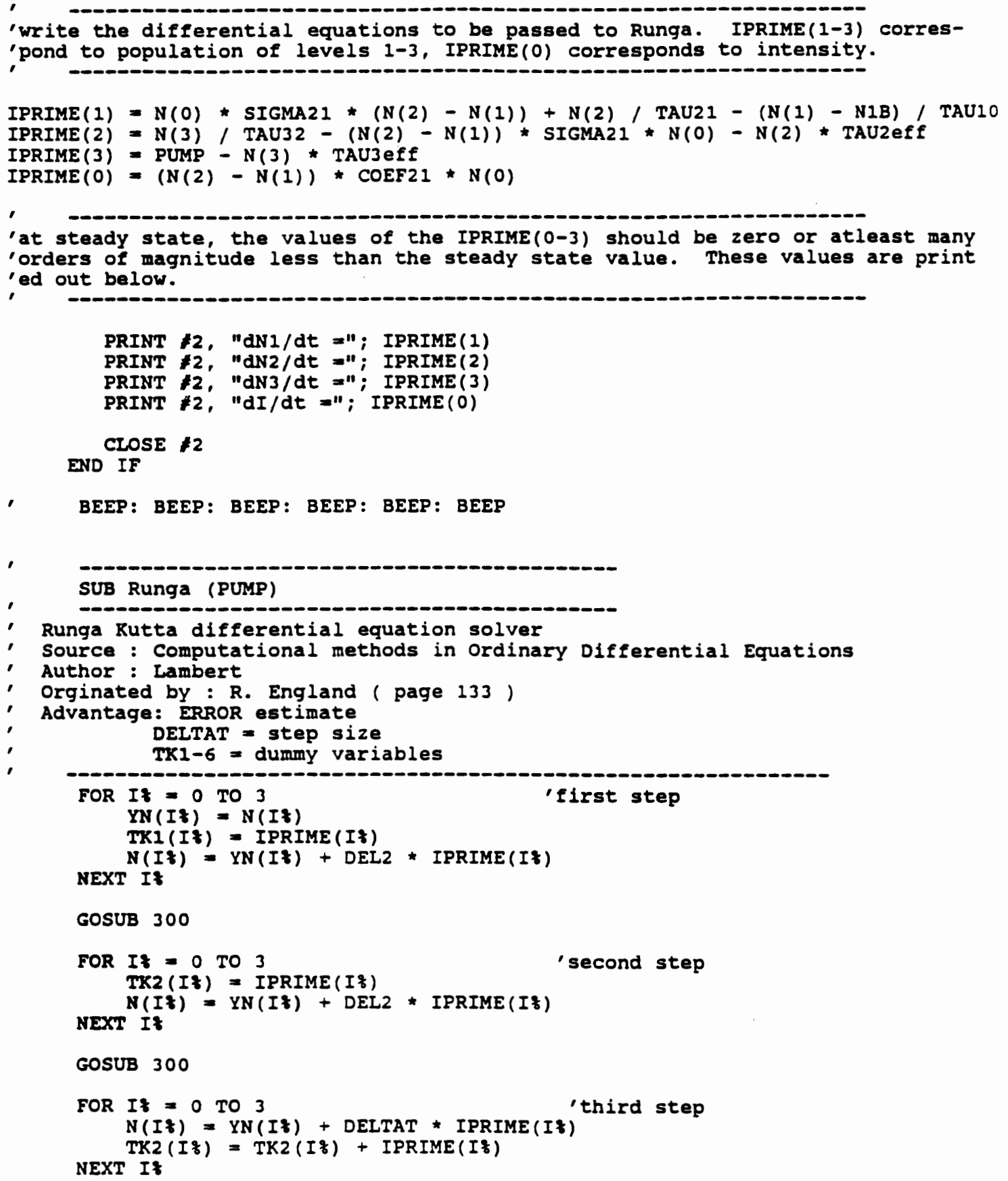


GOSUB 300

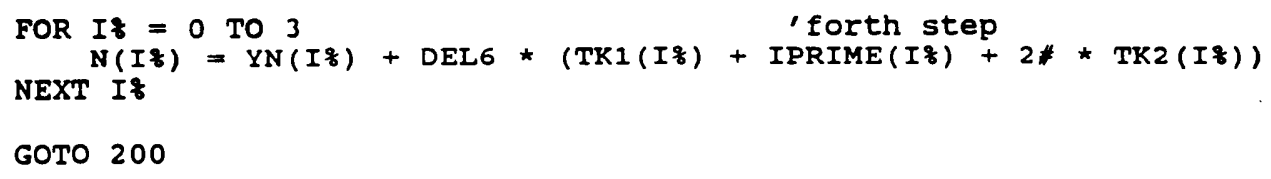

